Supporting Information

\title{
Benzaldehyde- and Nickel-Catalyzed Photoredox C(sp3)-H Alkylation/Arylation with Amides and Thioethers
}

Xiaojia Si, Lumin Zhang, A. Stephen K. Hashmi* 


\section{Table of Contents}

I. General information............................................2

II. General procedure and data characterization..................3

III. Large scale experiment.......................................4

IV. References..................................................24

V. $\quad$ NMR spectra..............................................25 


\section{General Information}

All the reactions were performed under nitrogen atmosphere. Chemicals (Aldrich, Fluka, TCI, Alfa, Abcr, and Merck) were used without further purification. Solvents were obtained from dried machine. NMR spectra were recorded on Bruker, Avance 300 (300 MHz), Avance 400 (400 MHz) and Avance 500 (500 MHz) spectrometers. Chemical shifts were referenced to residual solvent protons or TMS as internal standard and reported in ppm. The following abbreviations were used for ${ }^{1} \mathrm{H}$ NMR spectra to indicate the signal multiplicity: s (singlet); brs (broad singlet), $\mathrm{d}$ (doublet), $\mathrm{t}$ (triplet), q (quartet), quint (quintet), sext (sextet), sept (septet) and m (multiplet) as well as combinations of them. All ${ }^{13} \mathrm{C}$ NMR spectra were measured with ${ }^{1} \mathrm{H}$-decoupling. The multiplicities mentioned in these spectra [s (singlet, quaternary carbon), d (doublet, $\mathrm{CH}$-group), $\mathrm{t}$ (triplet, $\mathrm{CH}_{2}$-group), q (quartet, $\mathrm{CH}_{3}$-group)] were determined by DEPT-135 or HSQC spectra. For fluorine containing compounds, the second multiplicity refers to the ${ }^{19}$ F-coupling. IR spectra were recorded on a Bruker Vector 22, and the absorption maxima were given in wavelength in $\mathrm{cm}^{-1}$ units. Thin-layer chromatography (TLC) was performed on precoated polyester sheets (POLYGRAM SIL /GUV254), and components were visualized by observation under UV light or by treating the plates with vanillian/sulfuric acid solution followed by heating.

\section{Reaction equipment}

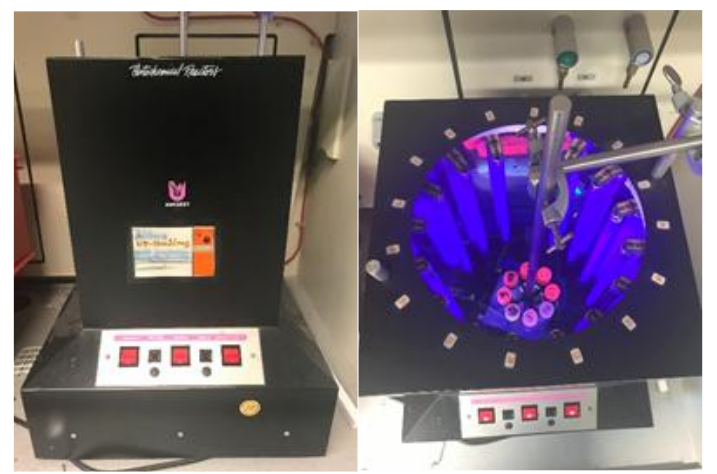

The photoreactor is equipped with one magnetic stirring machine and a fan at the bottom to keep the internal temperature around $31^{\circ} \mathrm{C}$ during the reaction processes. 


\section{General procedure for the photoredox $s p^{3}$ C-H alkylation/arylation}

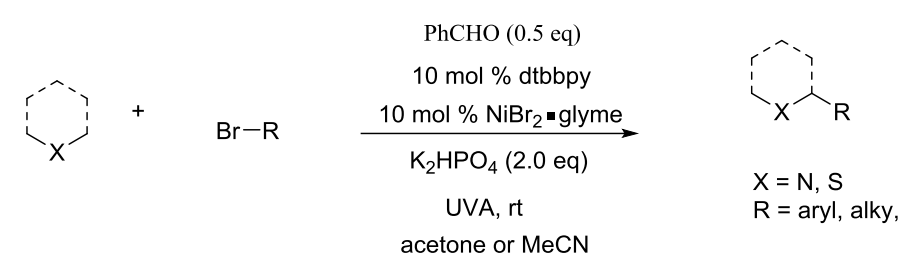

In a $10 \mathrm{~mL}$ dried pyrex screw-top reaction tube with suitable size stir bar, $\mathrm{K}_{2} \mathrm{HPO}_{4}$ (0.4 mmol, 2.0 equiv) were added. In another flask, $\mathrm{NiBr}_{2} \bullet$ glyme $(0.02 \mathrm{mmol}, 10$ mol\%), dtbbpy (0.02 mmol, $10 \mathrm{~mol} \%$ ) were dissolved in $0.3 \mathrm{ml}$ of acetone (for aryl bromides) or acetonitrile (for alkyl bromides) under $\mathrm{N}_{2}$, then put in the ultrasonic sink for 2-3 minutes until the solution turned to homogeneous. Add the nickel catalyst solution to the reaction tube with syringe, followed by alkyl or aryl bromides $(0.2$ mmol, 1.0 equiv), benzaldehyde ( $0.1 \mathrm{mmol}, 0.5$ equiv) and amides or thioethers (1.0 mmol, 5.0 equiv). The reaction mixture was then cooled to $-78{ }^{\circ} \mathrm{C}$ and degassed via vacuum evacuation (5 min), backfilled with nitrogen, and warmed to room temperature. This process was repeated three times. After the reaction was thoroughly degassed, irradiation of the reaction tubes was conducted with UVA lights for $72 \mathrm{~h}$. When the reaction finished, the reaction mixture was diluted with DCM, filtered to remove the inorganic base. The combined organic phase were dried over $\mathrm{MgSO}_{4}$ and filtered. After removal of the solvent in vacuo, the resulting crude products were subjected to flash column chromatography on silica gel to give desired product. The products were characterized with IR, ${ }^{1} \mathrm{H}$ NMR, ${ }^{13} \mathrm{C}$ NMR, ${ }^{19} \mathrm{~F}$ NMR and HRMS. 


\section{Large scale experiment}

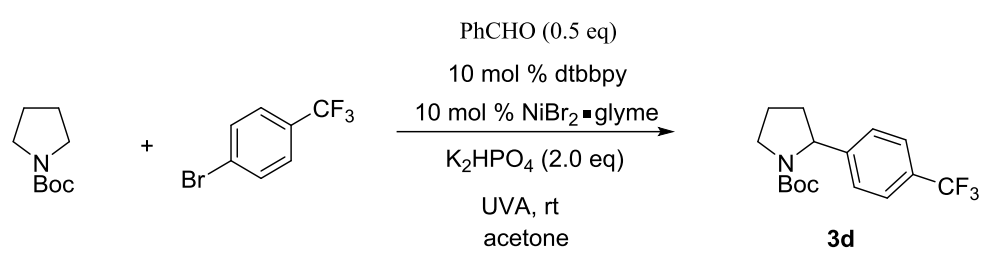

In a $10 \mathrm{~mL}$ dried pyrex screw-top reaction tube with suitable size stir bar, $\mathrm{K}_{2} \mathrm{HPO}_{4}$ (2.0 mmol, 2.0 equiv) were added. In another flask, $\mathrm{NiBr}_{2} \bullet$ glyme $(0.1 \mathrm{mmol}, 10$ mol \%), dtbbpy (0.1 mmol, $10 \mathrm{~mol} \%)$ were dissolved in $1.5 \mathrm{ml}$ of acetone under $\mathrm{N}_{2}$ protected, then put in the ultrasonic sink for 3 minutes until the solution turned to homogeneous. Add the nickel catalyst solution to the reaction tube with syringe, followed by 1-bromo-4-(trifluoromethyl)benzene (1.0 mmol, 1.0 equiv), benzaldehyde ( $0.5 \mathrm{mmol}, 0.5$ equiv) and tert-butyl pyrrolidine-1-carboxylate (5.0 mmol, 5.0 equiv). The reaction mixture was then cooled to $-78^{\circ} \mathrm{C}$ and degassed via vacuum evacuation (5 min), backfilled with nitrogen, and warmed to room temperature. This process was repeated three times. After the reaction was thoroughly degassed, irradiation of the reaction tubes was conducted with UVA lights for $300 \mathrm{~h}$. When the reaction finished, the reaction mixture was diluted with DCM, filtered to remove the inorganic base. The combined organic phase were dried over $\mathrm{MgSO}_{4}$ and filtered. After removal of the solvent in vacuo, the resulting crude products were subjected to flash column chromatography on silica gel to give desired product $\mathbf{3 d}$ $163.9 \mathrm{mg}$, yield 52\%. 


\section{Characterization data of the coupling products}

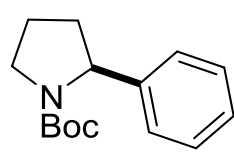

tert-Butyl-2-phenylpyrrolidine-1-carboxylate $( \pm)$

3a Colorless oil, $39.6 \mathrm{mg}, 80 \%$ yield, mixture of rotamers $(2.4: 1)$.

$\mathrm{R}_{\mathrm{f}}=0.3(\mathrm{DCM})$, chromatography eluent: $20 \%$ DCM in petroleum ether to DCM.

IR (Reflection): $\tilde{v}=3441,3029,2974,2930,2876,1692,1628,1478,1453,1389$, 1364, 1248, 1158, 1112, 1032, 900, 758, 700; ${ }^{\mathbf{1}} \mathbf{H}$ NMR $\left(300 \mathrm{MHz}, \mathrm{CDCl}_{3}\right): \delta=$ 7.25-7.15 (m, 2H), 7.15-7.04 (m, 3H), 4.98-4.55 (m, 1H), 3.64-3.36 (m, 2H), 2.34-2.09 (m, 1H), 1.91-1.62 (m, 3H), $1.37(\mathrm{~m}, 3 \mathrm{H}), 1.10(\mathrm{~m}, 6 \mathrm{H}) .{ }^{13} \mathrm{C}$ NMR $(75$ $\mathrm{MHz}, \mathrm{CDCl}_{3}$ ): $\delta=154.5$ (s), 145.2 (s), 128.1 (d), 126.5 (d), 125.5 (d), 79.1 (s), 61.3 (d), 60.7 (d), $47.1(\mathrm{t}), 36.0$ (t), 28.3 (q), 28.1 (q), 23.2 (t); HRMS (ESI): Calcd for $\mathrm{C}_{15} \mathrm{H}_{21} \mathrm{NNaO}_{2}[\mathrm{M}+\mathrm{Na}]: 270.1464$, found: 270.1465 .

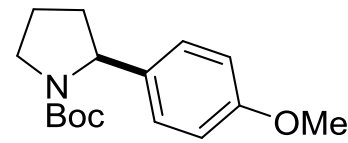

tert-Butyl-2-(4-methoxyphenyl)pyrrolidine-1-carboxylate $( \pm)$

3b Colorless oil, $43.3 \mathrm{mg}, 78 \%$ yield, mixture of rotamers (2:1).

$\mathrm{R}_{\mathrm{f}}=0.2(\mathrm{DCM})$, chromatography eluent: $20 \% \mathrm{DCM}$ in petroleum ether to DCM.

IR (Reflection): $\tilde{v}=2973,2932,2876,2836,1698,1612,1586$

$1513,1456,1395,1365,1302,1288,1248,1164,1113,1079,1036,971,900,875$, 827, 769; ${ }^{1} \mathbf{H}$ NMR $\left(500 \mathrm{MHz}, \mathrm{CDCl}_{3}\right): \delta=7.08(\mathrm{~d}, J=7.6 \mathrm{~Hz}, 2 \mathrm{H}), 6.83(\mathrm{~d}, J=8.6$ $\mathrm{Hz}, 2 \mathrm{H}), 4.95-4.68(\mathrm{~m}, 1 \mathrm{H}), 3.79(\mathrm{~s}, 3 \mathrm{H}), 3.65-3.42(\mathrm{~m}, 2 \mathrm{H}), 2.34-1.18(\mathrm{~m}, 1 \mathrm{H})$, 
1.95-1.75 (m, 3H), $1.44(\mathrm{~s}, 3 \mathrm{H}), 1.20(\mathrm{~s}, 6 \mathrm{H}) ;{ }^{13} \mathbf{C ~ N M R}\left(126 \mathrm{MHz}, \mathrm{CDCl}_{3}\right): \delta=158.3$ (s), 154.8 (s), 137.4 (s), 136.4 (s), 126.7 (d), 126.5 (d), 113.9 (d), 113.6 (d), 79.2 (s), $60.9(\mathrm{~d}), 60.2(\mathrm{~d}), 55.4(\mathrm{q}), 47.4(\mathrm{t}), 47.1(\mathrm{t}), 36.2(\mathrm{~d}), 35.0(\mathrm{~d}), 28.7(\mathrm{q}), 28.3(\mathrm{q}), 23.6$ (t), 23.3 (t); HRMS (ESI): Calcd for $\mathrm{C}_{16} \mathrm{H}_{23} \mathrm{NNaO}_{3}$ [M+Na]: 300.1570, found: 300.1572 .

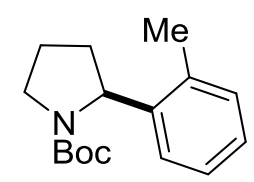

tert-Butyl-2-(o-tolyl)pyrrolidine-1-carboxylate $( \pm)$

3c Colorless oil, $35.5 \mathrm{mg}, 68 \%$ yield, mixture of rotamers (2.3:1).

$\mathrm{R}_{\mathrm{f}}=0.2(50 \% \mathrm{DCM} / \mathrm{PE})$, chromatography eluent: $10 \%$ to $50 \% \mathrm{DCM}$ in petroleum

IR (Reflection): $\tilde{v}=2973,2930,2874,1698,1605,1479,1456,1393,1365,1275$, 1247, 1159, 1120, 1102, 1079, 1051, 971, 921, 901, 875, 752, 725; ${ }^{\mathbf{1}} \mathbf{H}$ NMR (400 $\left.\mathrm{MHz}, \mathrm{CDCl}_{3}\right): \delta=7.16-6.98(\mathrm{~m}, 4 \mathrm{H}), 5.15-4.89(\mathrm{~m}, 1 \mathrm{H}), 3.72-3.46(\mathrm{~m}, 2 \mathrm{H})$, 2.39-2.19 (m, 4H), 1.77-2.02 (m, 3H), 1.46 (s, 3H), 1.14 (s, 6H); ${ }^{13}$ C NMR (101 MHz, $\left.\mathrm{CDCl}_{3}\right): \delta=154.8(\mathrm{~s}), 154.5$ (s), 143.4 (s), 142.1 (s), 134.2 (s), 134.0 (s), 130.7 (s), 130.1 (d), 126.5 (d), 126.4 (d), 126.0 (d), 124.6 (d), 124.1 (d), 79.1 (s), 79.0 (s), 58.2 (d), $47.5(\mathrm{t}), 47.2$ (t), 34.3 (t), 33.0 (t), 28.7 (q), 28.2 (q), 23.3 (t), 19.4 (q); HRMS (ESI): Calcd for $\mathrm{C}_{16} \mathrm{H}_{23} \mathrm{NNaO}_{2}$ [M+Na]: 284.1621, found: 284.1621 .

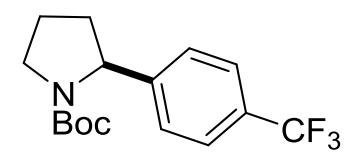

tert-Butyl-2-(4-(trifluoromethyl)phenyl)pyrrolidine-1-carboxylate $( \pm)$ 
3d Colorless oil, $53.6 \mathrm{mg}, 85 \%$ yield, mixture of rotamers (1.7:1).

$\mathrm{R}_{\mathrm{f}}=0.4$ (DCM), chromatography eluent: $20 \%$ to $80 \%$ DCM in petroleum ether.

IR (Reflection): $\tilde{v}=2977,2933,2880,1696,1619,1479,1455,1391,1366,1324$, 1256, 1158, 1117, 1067, 1016, 902, 834; ${ }^{1} \mathbf{H}$ NMR (500 MHz, $\left.\mathrm{CDCl}_{3}\right): \delta=7.55(\mathrm{~d}, J$ $=8.1 \mathrm{~Hz}, 2 \mathrm{H}), 7.28(\mathrm{~d}, J=8.1 \mathrm{~Hz}, 2 \mathrm{H}), 5.01-4.75(\mathrm{~m}, 1 \mathrm{H}), 3.69-3.47(\mathrm{~m}, 2 \mathrm{H})$, 2.42-2.25 (m, 1H), 1.95-1.74 (m, 3H), $1.45(\mathrm{~s}, 3 \mathrm{H}), 1.18(\mathrm{~m}, 6 \mathrm{H}) ;{ }^{13} \mathbf{C}$ NMR (101 $\mathrm{MHz}, \mathrm{CDCl}_{3}$ ): $\delta=154.6$ (s), 149.4 (s), 148.4 (s), 129.0 (q, $\left.J=32.3 \mathrm{~Hz}, \mathrm{~s}\right), 125.9$ (d), 125.3 (d), 123.0 (q, $J=340.2 \mathrm{~Hz}, \mathrm{~s}), 115.6$ (s), 79.7 (s), 61.2 (d), 60.7 (d), 47.5 (t), $47.3(\mathrm{t}), 36.1(\mathrm{t}), 34.9$ (t), 28.6 (q), 28.3 (q), $23.7(\mathrm{t}), 23.3(\mathrm{t}) ;{ }^{19} \mathbf{F}$ NMR (283 MHz, $\left.\mathrm{CDCl}_{3}\right): \delta=-61.4,-62.3,-62.4,-63.2 ;$ HRMS (ESI): Calcd for $\mathrm{C}_{16} \mathrm{H}_{20} \mathrm{~F}_{3} \mathrm{NNaO}_{2}$ [M+Na]: 338.1338, found: 338.1337.

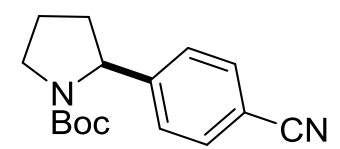

tert-Butyl-2-(4-cyanophenyl)pyrrolidine-1-carboxylate $( \pm)$

3e Colorless oil, $39.2 \mathrm{mg}$, 72\% yield, mixture of rotamer (1.6:1).

$\mathrm{R}_{\mathrm{f}}=0.3$ (DCM), chromatography eluent: $20 \%$ to $80 \%$ DCM in petroleum ether.

IR (Reflection): $\tilde{v}=2973,2933,2879,2227,1687,1608,1507,1475,1456,1396$, 1368, 1353, 1309, 1280, 1247, 1156, 1110, 1016, 903, 854, 842, 774; ${ }^{1}$ H NMR (400 $\left.\left.\mathrm{MHz}_{\mathrm{CDCl}}\right)\right): \delta=7.60(\mathrm{~d}, J=8.3 \mathrm{~Hz}, 2 \mathrm{H}), 7.28(\mathrm{~d}, J=8.3 \mathrm{~Hz}, 2 \mathrm{H}), 5.00-4.70(\mathrm{~m}$, 1H), 3.71-3.49 (m, 2H), 2.44-2.23 (m, 1H), 1.94-1.70 (m, 3H), $1.44(\mathrm{~s}, 3 \mathrm{H}), 1.18(\mathrm{~s}$, 6H). ${ }^{13}$ C NMR (101 MHz, $\left.\mathrm{CDCl}_{3}\right): \delta=154.4$ (s), 151.0 (s), 150.0 (s), 134.2 (d), 132.3 (d), 126.4 (d), 119.1 (d), 116.5 (s), 110.6 (s), 79.9 (s), 61.3 (d), 60.8 (d), 47.4 (t), 36.1 (t), 34.9 (t), 28.6 (q), 28.3 (q), 23.8 (t), 23.4 (t); HRMS (ESI): Calcd for $\mathrm{C}_{16} \mathrm{H}_{20} \mathrm{~N}_{2} \mathrm{NaO}_{2}$ [M+Na]: 295.1417, found: 295.1415.

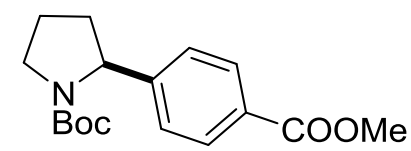


tert-Butyl-2-(4-(methoxycarbonyl)phenyl)pyrrolidine-1-carboxylate $( \pm)$

3f Colorless oil, $34.8 \mathrm{mg}, 57 \%$ yield, mixture of rotamer (2.2:1).

$\mathrm{R}_{\mathrm{f}}=0.2(\mathrm{DCM})$, chromatography eluent: $20 \%$ DCM in petroleum ether to DCM.

IR (Reflection): $\tilde{v}=2974,2878,1933,1724,1688,1611,1577,1479,1436,1398$, 1366, 1283, 1167, 1116, 1018, 969, 902, 874, 857, 829, 772, 706; ${ }^{1} \mathbf{H}$ NMR (400 MHz, $\left.\mathrm{CDCl}_{3}\right): \delta=7.97(\mathrm{~d}, J=8.4 \mathrm{~Hz}, 2 \mathrm{H}), 7.23(\mathrm{~d}, J=8.4 \mathrm{~Hz}, 2 \mathrm{H}), 5.08-4.66(\mathrm{~m}, 1 \mathrm{H})$, $3.90(\mathrm{~s}, 3 \mathrm{H}), 3.72-3.46(\mathrm{~m}, 2 \mathrm{H}), 2.41-2.21(\mathrm{~m}, 1 \mathrm{H}), 1.97-1.71(\mathrm{~m}, 3 \mathrm{H}), 1.44(\mathrm{~s}, 3 \mathrm{H})$, 1.16 (s, 6H). ${ }^{13} \mathrm{C}$ NMR (101 MHz, $\left.\mathrm{CDCl}_{3}\right): \delta=167.2$ (s), 154.6 (s), 150.8 (s), 149.7 (s), $129.8(\mathrm{~d}), 128.7(\mathrm{~s}), 125.6(\mathrm{~d}), 79.6(\mathrm{~s}), 61.4(\mathrm{~d}), 60.8(\mathrm{~d}), 52.1(\mathrm{q}), 47.5(\mathrm{t}), 47.3(\mathrm{t})$ $36.1(\mathrm{t}), 34.9$ (t), 28.6 (q), 28.3 (q), $23.7(\mathrm{t}), 23.4(\mathrm{t})$; HRMS (ESI): Calcd for $\mathrm{C}_{34} \mathrm{H}_{46} \mathrm{~N}_{2} \mathrm{NaO}_{8}$ [2M+Na]: 633.3146, found: 633.3147.

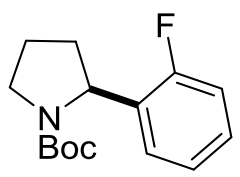

tert-Butyl-2-(2-fluorophenyl)pyrrolidine-1-carboxylate $( \pm)$

$3 \mathbf{g}$ Colorless oil, $31.8 \mathrm{mg}, 60 \%$ yield, mixture of rotamer (2.3:1).

$R_{f}=0.2(D C M)$, chromatography eluent: $20 \%$ DCM in petroleum ether to DCM.

IR (Reflection): $\tilde{v}=3067,2975,2931,2877,1702,1588,1487,1456,1396,1366$, 1274, 1246, 1224, 1161, 1118, 1097, 1035, 973, 918, 903, 876, 821, 756; ${ }^{1} \mathbf{H}$ NMR $\left(500 \mathrm{MHz}, \mathrm{CDCl}_{3}\right): \delta=7.22-7.04(\mathrm{~m}, 3 \mathrm{H}), 7.00(\mathrm{dd}, J=10.2,8.6 \mathrm{~Hz}, 1 \mathrm{H}), 5.23-5.01$ (m, 1H), 3.66-3.54 (m, 2H), 2.40-2.24 (m, 1H), 1.93-1.78 (m, 3H), 1.46 (s, 3H), 1.19 (s, 6H). ${ }^{13} \mathrm{C}$ NMR (126 MHz, $\left.\mathrm{CDCl}_{3}\right): \delta=160.9$ (s), 159.0 .6 (s), 154.5 (s), 132.0.7 (d, 
$J=13.7 \mathrm{~Hz}, \mathrm{~s}), 128.1(\mathrm{~d}, J=8.1 \mathrm{~Hz}, \mathrm{~d}), 127.1(\mathrm{~d}, J=3.4 \mathrm{~Hz}, \mathrm{~d}), 123.9$ (d, $J=2.9 \mathrm{~Hz}$, d), $115.2(\mathrm{~d}, J=21.8 \mathrm{~Hz}, \mathrm{~d}), 79.5(\mathrm{~s}), 55.6(\mathrm{~d}), 55.3(\mathrm{~d}), 47.3(\mathrm{q}), 47.0$ (t) 34.6 (t), 33.5 (t), 28.6 (q), 28.2 (q), 23.7 (t), 23.4 (t); ${ }^{19}$ F NMR (283 MHz, $\left.\mathrm{CDCl}_{3}\right): \delta=-108.9$, -109.4, -118.63, -119.94; HRMS (ESI): Calcd for $\mathrm{C}_{15} \mathrm{H}_{20} \mathrm{FNNaO}_{2}$ [M+Na]: 288.1370, found: 288.1373 .

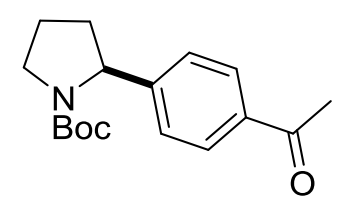

tert-Butyl-2-(4-acetylphenyl)pyrrolidine-1-carboxylate $( \pm)$

3h Colorless oil, $25.1 \mathrm{mg}, 43 \%$ yield, mixture of rotamers (1.9:1).

$\mathrm{R}_{\mathrm{f}}=0.2(\mathrm{DCM})$, chromatography eluent: $20 \%$ DCM in petroleum ether to DCM.

IR (Reflection): $\tilde{v}=2975,2929,2877,1683,1607,1478,1454,1391,1364,1267$, 1160, 1112, 1015, 957, 830; ${ }^{1} \mathbf{H}$ NMR (500 MHz, $\left.\mathrm{CDCl}_{3}\right): \delta=7.90(\mathrm{~d}, J=8.3 \mathrm{~Hz}$, 2H), $7.26(\mathrm{~d}, J=8.3 \mathrm{~Hz}, 2 \mathrm{H}), 5.02-4.76(\mathrm{~m}, 1 \mathrm{H}), 3.69-3.50(\mathrm{~m}, 2 \mathrm{H}), 2.59(\mathrm{~s}, 3 \mathrm{H})$, 2.41-2.26 (m, 1H), 1.95-1.76 (m, 3H), 1.45 (s, 3H), $1.17(\mathrm{~s}, 6 \mathrm{H}) ;{ }^{13} \mathbf{C}$ NMR (75 MHz, $\mathrm{CDCl}_{3}$ ): $\delta=197.8$ (s), 174.4 (s), 154.5 (s), 150.9 (s), 149.9 (s), 135.8 (s), 128.5 (d), $125.7(\mathrm{~d}), 79.6(\mathrm{~s}), 61.2(\mathrm{~d}), 60.8(\mathrm{~d}), 47.5(\mathrm{t}), 47.2$ (t) 36.0 (t), 34.9 (t), 28.6 (q), 28.2 (q), 26.7 (q), 23.7 (t), 23.4 (t); HRMS (ESI): Calcd for $\mathrm{C}_{17} \mathrm{H}_{23} \mathrm{NNaO}_{3}[\mathrm{M}+\mathrm{Na}]$ : 312.1570, found: 312.1572 .

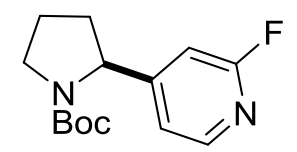

tert-Butyl-2-(2-fluoropyridin-4-yl)pyrrolidine-1-carboxylate $( \pm)$

3i Colorless oil, $11.2 \mathrm{mg}, 21 \%$ yield, mixture of rotamers (1.7:1). 
$\mathrm{R}_{\mathrm{f}}=0.4(1 \% \mathrm{MeOH} / \mathrm{DCM})$, chromatography eluent: DCM to $1 \% \mathrm{MeOH}$ in DCM.

IR (Reflection): $\tilde{v}=2976,2933,2880,1698,1611,1572,1479,1454,1392,1367$, 1280, 1256, 1163, 1113, 975, 949, 867, 773, 666; ${ }^{1} \mathbf{H}$ NMR $\left(400 \mathrm{MHz}, \mathrm{CDCl}_{3}\right): \delta=$ $8.13(\mathrm{~d}, J=5.0 \mathrm{~Hz}, 1 \mathrm{H}), 7.00(\mathrm{dt}, J=5.2,1.5 \mathrm{~Hz}, 1 \mathrm{H}), 6.74(\mathrm{~s}, 1 \mathrm{H}), 4.97-4.70(\mathrm{~m}$, $1 \mathrm{H}), 3.71-3.50(\mathrm{~m}, 2 \mathrm{H}), 2.45-2.26(\mathrm{~m}, 1 \mathrm{H}), 1.95-1.74(\mathrm{~m}, 3 \mathrm{H}), 1.46(\mathrm{~s}, 3 \mathrm{H}), 1.23(\mathrm{~s}$, 6H); ${ }^{13}$ C NMR (101 MHz, $\left.\mathrm{CDCl}_{3}\right): \delta=165.5$ (s), $163.2(\mathrm{~s}), 154.7$ (s), 154.3 (s), 147.7 (d, $J=15.3 \mathrm{~Hz}, \mathrm{~d}), 118.7$ (d), 106.4 (d, $J=38.1 \mathrm{~Hz}, \mathrm{~d}$ ), 80.2 (s), 60.5 (d), 60.0 (d), $47.4(\mathrm{t}), 47.3(\mathrm{t}), 35.5(\mathrm{t}), 34.3(\mathrm{t}), 28.6(\mathrm{q}), 28.3(\mathrm{q}), 23.8(\mathrm{t}), 23.4(\mathrm{t}) ;{ }^{19} \mathbf{F}$ NMR (283 $\mathrm{MHz}, \mathrm{CDCl}_{3}$ ): $\delta=-68.5$; HRMS (ESI): Calcd for $\mathrm{C}_{14} \mathrm{H}_{19} \mathrm{FN}_{2} \mathrm{NaO}_{2}$ [M+Na]: 289.1323, found: 289.1321 .

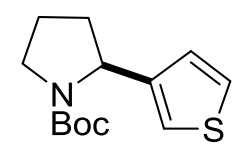

tert-Butyl-2-(thiophen-3-yl)pyrrolidine-1-carboxylate $( \pm)$

3j Colorless oil, $16.2 \mathrm{mg}, 32 \%$ yield, mixture of rotamers (1.7:1).

$\mathrm{R}_{\mathrm{f}}=0.6$ (DCM), chromatography eluent: petroleum to 50\% DCM in petroleum ether.

IR (Reflection): $\tilde{v}=3099,2973,2930,2877,1696,1478,1454,1393,1366,1326$, 1258, 1169, 1111, 1021, 975, 917, 858, 797, 776, 687, 647; ${ }^{1}$ H NMR $(600 \mathrm{MHz}$, $\left.\mathrm{CDCl}_{3}\right): \delta=7.23(\mathrm{dd}, J=4.9,3.0 \mathrm{~Hz}, 1 \mathrm{H}), 7.03-6.87(\mathrm{~m}, 2 \mathrm{H}), 5.12-4.80(\mathrm{~m}, 1 \mathrm{H})$, 3.62-3.37 (m, 2H), 2.28-2.11 (m, 1H), 1.96-1.81 (m, 3H), $1.46(\mathrm{~s}, 3 \mathrm{H}), 1.27(\mathrm{~s}, 6 \mathrm{H})$; ${ }^{13} \mathrm{C}$ NMR (151 MHz, $\mathrm{CDCl}_{3}$ ): $\delta=154.7$ (s), 146.1 (s), 145.2 (s), 126.1 (d), 126.0 (d), 125.8 (d), 125.6 (d), 119.7 (d), 79.4 (s), 57.4 (d), 57.0 (d), $46.8(\mathrm{t}), 46.5(\mathrm{t}), 34.9$ (t), 33.5 (t), 28.7 (q), 28.4 (q), 23.9 (t), 23.4 (t); HRMS (ESI): Calcd for $\mathrm{C}_{13} \mathrm{H}_{19} \mathrm{NNaO}_{2} \mathrm{~S}$ [M+Na]: 276.1029, found: 276.1031 .

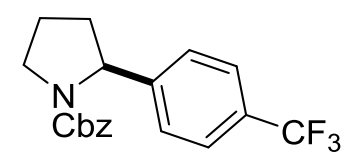


Benzyl-2-(4-(trifluoromethyl)phenyl)pyrrolidine-1-carboxylate $( \pm)$

3k Colorless oil, $59.4 \mathrm{mg}, 85 \%$ yield.

$\mathrm{R}_{\mathrm{f}}=0.3(50 \% \mathrm{DCM} / \mathrm{PE})$, chromatography eluent: $10 \%$ to $50 \% \mathrm{DCM}$ in petroleum ether.

IR (Reflection): $\tilde{v}=3066,3034,2975,2954,2880,1702,1619$

1498, 1449, 1410, 1352, 1325, 1273, 1211, 1162, 1116, 1067, 1016, 980, 914, 831, 771, 734, 696, 649, 609; ${ }^{1} \mathbf{H}$ NMR (300 MHz, $\left.\mathrm{CDCl}_{3}\right): \delta=7.53-7.40(\mathrm{~m}, 2 \mathrm{H})$, 7.38-7.00 (m, 6H), 6.69-6.84 (m, 1H), 5.17-4.71 (m, 3H), 3.73-3.47 (m, 2H), 2.44-2.14 (m, 1H), $1.94-1.67(\mathrm{~m}, 3 \mathrm{H}) ;{ }^{13} \mathrm{C} \mathrm{NMR}\left(75 \mathrm{MHz}, \mathrm{CDCl}_{3}\right): \delta=155.0(\mathrm{~s})$, 148.6 (s), 147.8 (s), 136.9 (s), 136.5 (s), 129.2 (q, $J=32.3 \mathrm{~Hz}, \mathrm{~s}), 128.6$ (d), 128.3 (d), 128.1 (d), 127.9 (d), 127.6 (d), 126.0 (d), 125.6 (d), 124.3 .0 (q, $J=340.2 \mathrm{~Hz}, \mathrm{~s}), 115.5$ (d), $67.1(\mathrm{t}), 67.0(\mathrm{t}), 61.2(\mathrm{~d}), 61.0(\mathrm{~d}), 47.9(\mathrm{t}), 47.4(\mathrm{t}) 36.0(\mathrm{t}), 34.8(\mathrm{t}), 23.8(\mathrm{t}), 23.2$ (t); ${ }^{19}$ F NMR (283 MHz, $\left.\mathrm{CDCl}_{3}\right): \delta=-61.4,-62.4,-62.5$; HRMS (ESI): Calcd for $\mathrm{C}_{19} \mathrm{H}_{18} \mathrm{~F}_{3} \mathrm{NNaO}_{2}[\mathrm{M}+\mathrm{Na}]: 372.1182$, found: 372.1184 .

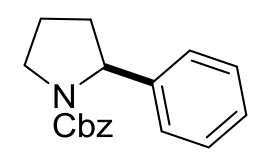

Benzyl-2-phenylpyrrolidine-1-carboxylate $( \pm)$

31 Colorless oil, $41.6 \mathrm{mg}, 74 \%$ yield.

$\mathrm{R}_{\mathrm{f}}=0.6(\mathrm{DCM})$, chromatography eluent: petroleum to $50 \% \mathrm{DCM}$ in petroleum ether.

IR (Reflection): $\tilde{v}=3087,3062,3030,2973,2952,2878,1952,1876,1713,1603$, 1586, 1540, 1496, 1451, 1416, 1354, 1272, 1244, 1210, 1192, 1173, 1111, 1075, 1028, 
980, 955, 911, 873, 850, 769, 751, 697, 635, 606; ${ }^{1} \mathbf{H}$ NMR (300 MHz, $\left.\mathrm{CDCl}_{3}\right): \delta=$ 7.49-7.09 (m, 9H), 7.01-6.81 (m, 1H), 5.27-4.89 (m, 3H), 3.82-3.55 (m, 2H), 2.46-2.21 (m, 1H), 2.05-1.85 (m, 3H); $\left.{ }^{13} \mathbf{C ~ N M R ~ ( 7 5 ~ M H z , ~} \mathrm{CDCl}_{3}\right): \delta=155.1(\mathrm{~s})$, 155.0 (s), 144.4 (s), 143.6 (s), 137.2 (s), 136.8 (s), 128.5 (d), 128.2 (d), 128.0 (d), $127.6(\mathrm{~d}), 127.3(\mathrm{~d}), 126.8(\mathrm{~d}), 125.6(\mathrm{~d}), 66.8(\mathrm{t}), 66.6(\mathrm{t}), 61.4(\mathrm{~d}), 61.2(\mathrm{~d}), 47.7(\mathrm{t})$, $47.3(t), 36.0(t), 34.8(t), 23.7(t), 23.0(t)$; HRMS (ESI): Calcd for $\mathrm{C}_{18} \mathrm{H}_{19} \mathrm{NNaO}_{2}$ [M+Na]: 304.1308, found: 304.1312.

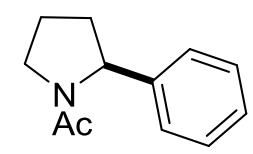

1-(2-phenylpyrrolidin-1-yl)Ethan-1-one ( \pm )

3m Colorless oil, $9.4 \mathrm{mg}, 25 \%$ yield, mixture of rotamers (2.8:1).

$\mathrm{R}_{\mathrm{f}}=0.2(30 \% \mathrm{DCM} / \mathrm{PE})$, chromatography eluent: petroleum ether to $30 \% \mathrm{DCM}$ in petroleum ether.

IR (Reflection): $\tilde{v}=3060,3028,2973,2877,1644,1494,1414,1354,1270,1210$, 1190, 1079, 1030, 989, 750, 702; ${ }^{1} \mathbf{H}$ NMR (300 MHz, $\left.\mathrm{CDCl}_{3}\right): \delta=7.40-7.12(\mathrm{~m}, 5 \mathrm{H})$, 5.27-4.88 (m, 1H), 3.84-3.54 (m, 2H), 2.47-2.20 (m, 1H), 2.17-1.81 (m, 6H); ${ }^{13} \mathrm{C}$ NMR (75 MHz, $\mathrm{CDCl}_{3}$ ): $\delta=170.4$ (s), 169.3 (s), 143.3 (s), 143.0 (s), 128.9 (d), 128.4 (d), 127.3 (d), 126.7 (d), 125.5 (d), 125.5 (d), 62.4 (d), 60.3 (d), 48.5 (t), 47.0 (t), 36.4 (t), $34.2(\mathrm{t}), 23.8(\mathrm{t}), 22.9$ (q), 22.7 (q), 22.0 (t); HRMS (EI): Calcd for $\mathrm{C}_{12} \mathrm{H}_{15} \mathrm{NO}$ $\left[\mathrm{M}^{\bullet+}\right]$ : 189.11482 , found: 189.11488 .

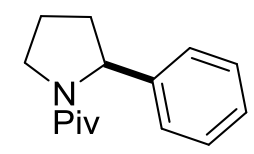

2,2-Dimethyl-1-(2-phenylpyrrolidin-1-yl)propan-1-one $( \pm)$ 
3n Colorless oil, $9.8 \mathrm{mg}, 21 \%$ yield.

$\mathrm{R}_{\mathrm{f}}=0.2(30 \% \mathrm{DCM} / \mathrm{PE})$, chromatography eluent: petroleum ether to $30 \% \mathrm{DCM}$ in petroleum ether.

IR (Reflection): $\tilde{v}=3061,3028,2969,2874,1947,1875,1804,1720,1626,1479$, $1450,1403,1375,1361,1206,1162,1077,1028,898,841,800,755,700,647 ;{ }^{1} \mathbf{H}$

NMR (300 MHz, $\left.\mathrm{CDCl}_{3}\right): \delta=7.32-7.24(\mathrm{~m}, 2 \mathrm{H}), 7.22-7.17(\mathrm{~m}, 1 \mathrm{H}), 7.17-7.09(\mathrm{~m}$, 2H), 5.25 (br. s, 1H), 3.95-3.69 (m, 2H), 2.31-2.14 (m, 1H), 2.02-1.71 (m, 3H), 1.26 (s, 9H); ${ }^{13}$ C NMR (75 MHz, $\mathrm{CDCl}_{3}$ ): $\delta=176.7$ (s), 144.4 (s), 128.5 (d), 126.6 (d), 125.3 (d), 62.4 (d), 48.9 (q), 39.4 (t), 33.6 (t), 27.9 (t); HRMS (ESI): Calcd for $\mathrm{C}_{15} \mathrm{H}_{21} \mathrm{NNaO}[\mathrm{M}+\mathrm{Na}]: 254.1515$, found: 254.1517 .<smiles>O=C(Cc1ccccc1)N1CCCC1c1ccccc1</smiles>

2-Phenyl-1-(2-phenylpyrrolidin-1-yl)ethan-1-one ( \pm )

3o Colorless oil, $31.8 \mathrm{mg}, 60 \%$ yield, mixture of rotamers (2.2:1).

$\mathrm{R}_{\mathrm{f}}=0.2$ (DCM), chromatography eluent: $20 \%$ DCM in petroleum ether to DCM.

IR (Reflection): $\tilde{v}=30601,3028,2972,2949,2876,1950,1881,1810,1649,1583$, 1495, 1453, 1416, 1355, 1297, 1242, 1186, 1076, 1030, 972, 894, 848, 753, 720, 699; ${ }^{1} \mathbf{H}$ NMR $\left(300 \mathrm{MHz}, \mathrm{CDCl}_{3}\right): \delta=7.33-7.06(\mathrm{~m}, 8 \mathrm{H}), 7.03-6.95(\mathrm{~m}, 2 \mathrm{H}), 5.20-4.82(\mathrm{~m}$, $1 \mathrm{H}), 3.81-3.48(\mathrm{~m}, 3 \mathrm{H}), 1.38(\mathrm{~s}, 1 \mathrm{H}), 2.32-2.07(\mathrm{~m}, 1 \mathrm{H}), 1.88-1.70(\mathrm{~m}, 3 \mathrm{H}) ;{ }^{13} \mathrm{C}$ NMR (126 MHz, $\left.\mathrm{CDCl}_{3}\right): \delta=170.6$ (s), 169.6 (s), 143.5 (s), 143.2 (s), 135.1 (s), 134.9 (s), 129.2 (d), 129.1 (d), 129.0 (d), 128.7 (d), 128.5 (d), 128.4 (d), 127.5 (d), $126.9(\mathrm{~d}), 126.7$ (d), $125.6(\mathrm{~d}), 125.6(\mathrm{~d}), 61.6(\mathrm{~d}), 60.7$ (d), $48.0(\mathrm{t}), 47.4(\mathrm{t}), 42.7(\mathrm{t})$, 41.8 (t), 36.4 (t), 34.1 (t), 23.9 (t), 21.7 (t); HRMS (ESI): Calcd for $\mathrm{C}_{18} \mathrm{H}_{19} \mathrm{NNaO}$ [M+Na]: 288.1359, found: 288.1361 . 
<smiles>CN(C)C(=O)N(C)Cc1ccccc1</smiles>

1-Benzyl-1,3,3-trimethylurea

3p Colorless oil, $20.4 \mathrm{mg}, 53 \%$ yield.

$\mathrm{R}_{\mathrm{f}}=0.1(\mathrm{DCM})$, chromatography eluent: $20 \%$ DCM in petroleum ether to DCM.

IR (Reflection): $\tilde{v}=3392,3086,3062,3028,3004,2925,1812,1644$

1496, 1453, 1384, 1259, 1177, 1121, 1062, 1026, 920, 798, 730, 699; ${ }^{1}$ H NMR (500

$\left.\mathrm{MHz}, \mathrm{CDCl}_{3}\right): \delta=7.37-7.26(\mathrm{~m}, 5 \mathrm{H}), 4.38(\mathrm{~s}, 2 \mathrm{H}), 2.85(\mathrm{~s}, 6 \mathrm{H}), 2.73(\mathrm{~s}, 3 \mathrm{H}) ;{ }^{13} \mathrm{C}$

NMR (126 MHz, $\mathrm{CDCl}_{3}$ ): $\delta=138.2$ (s), 128.7 (d), 127.8 (d), 127.3 (d), 125.8 (s),

54.3 (q), 38.9 (q), 36.7 (q); HRMS (EI): Calcd for $\mathrm{C}_{11} \mathrm{H}_{16} \mathrm{~N}_{2} \mathrm{O}\left[\mathrm{M}^{\cdot+}\right]$ : 192.12571, found: 192.12656 .<smiles>CN(Cc1ccc(C(F)(F)F)cc1)c1ccc(C#N)cc1</smiles>

4-(methyl(4-(trifluoromethyl)benzyl)amino)Benzonitrile

3q Colorless oil, $24.4 \mathrm{mg}, 42 \%$ yield.

$\mathrm{R}_{\mathrm{f}}=0.4(50 \% \mathrm{DCM} / \mathrm{PE})$, chromatography eluent: $10 \%$ to $50 \%$ DCM in petroleum.

IR (Reflection): $\tilde{v}=2912,2214,1610,1524,1481,1456,1418,1385,1329,1255$, 1216, 1180, 1125, 1067, 1017, 934, 817, 750, 703, 632; ${ }^{\mathbf{H}} \mathbf{H}$ NMR (400 MHz, $\left.\mathrm{CDCl}_{3}\right)$ : $\delta=7.59(\mathrm{~d}, J=8.1 \mathrm{~Hz}, 2 \mathrm{H}), 7.45(\mathrm{~d}, J=9.0 \mathrm{~Hz}, 2 \mathrm{H}), 7.28(\mathrm{~d}, J=8.1 \mathrm{~Hz}, 2 \mathrm{H}), 6.67(\mathrm{~d}$, $J=9.0 \mathrm{~Hz}, 2 \mathrm{H}), 4.66(\mathrm{~s}, 2 \mathrm{H}), 3.14(\mathrm{~s}, 3 \mathrm{H}) ;{ }^{13} \mathbf{C ~ N M R}\left(101 \mathrm{MHz}, \mathrm{CDCl}_{3}\right): \delta=151.9$ 
(s), 141.6 (s), 133.8 (d), 129.9 (q, J = 32.6, s), 129.3 (d), 128.8 (d), 126.7 (d), 126.0 (q, $J=3.8$, d), 120.4 (s), 115.8 (d), 111.9 (d), 98.7 (s), 55.8 (t), 39.0 (q); ${ }^{19}$ F NMR (283 $\mathrm{MHz}, \mathrm{CDCl}_{3}$ ): $\delta=-61.7,-62.5$; HRMS (ESI): Calcd for $\mathrm{C}_{16} \mathrm{H}_{13} \mathrm{~F}_{3} \mathrm{~N}_{2} \mathrm{Na}[\mathrm{M}+\mathrm{Na}]$ : 313.0923, found: 313.0923 .<smiles>CC(=O)N(C)Cc1ccc(C(F)(F)F)cc1</smiles>

$N$-methyl- $N$-(4-(trifluoromethyl)benzyl)acetamide

3r Colorless oil, $31.9 \mathrm{mg}, 69 \%$ yield.

$\mathrm{R}_{\mathrm{f}}=0.2(\mathrm{DCM})$, chromatography eluent: $20 \%$ DCM in petroleum ether to DCM.

IR (Reflection): $\tilde{v}=3470,3021,2932,2645,1928,1657,1485,1407,1360,1333$, $1273,1243,1219,1167,1134,1068,1017,985,935,820,770,752,721,644 ;{ }^{1} \mathbf{H}$ NMR $\left(400 \mathrm{MHz}, \mathrm{CDCl}_{3}\right): \delta=7.65-7.54(\mathrm{~m}, 2 \mathrm{H}), 7.37-7.26(\mathrm{~m}, 2 \mathrm{H}), 4.65-4.54(\mathrm{~m}$, 2H), 2.94 (s, 3H), 2.18-2.10 (m, 3H); $\left.{ }^{13} \mathbf{C ~ N M R ~ ( 1 0 1 ~ M H z , ~} \mathrm{CDCl}_{3}\right): \delta=171.1$ (s), 171.0 (s), 141.7 (s), 141.0 (s), 128.3 (d), 126.7 (d), 126.1 (q, $J=3.8, \mathrm{~d}), 125.7$ (q, $J=$ 3.8, d), 122.9 (s), 122.7 (s), 54.0 (t), 50.4 (t), 35.9 (q), 34.0 (q), 21.8 (q), 21.5 (q); ${ }^{19} \mathbf{F}$ NMR (283 MHz, $\left.\mathrm{CDCl}_{3}\right): \delta=-62.6,-62.6$; HRMS (ESI): Calcd for $\mathrm{C}_{11} \mathrm{H}_{12} \mathrm{~F}_{3} \mathrm{NNaO}$ [M+Na]: 254.0763, found: 254.0761.

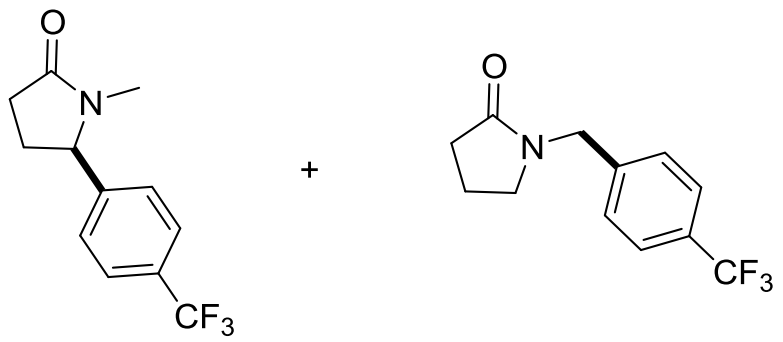

Methyl-5-(4-(trifluoromethyl)phenyl)pyrrolidin-2-one 3sa 
1-(4-(trifluoromethyl)benzyl)Pyrrolidin-2-one 3sb

Colorless oil, $38.9 \mathrm{mg}, 80 \%$ yield, 3sa:3sb (6.3:1).

$\mathrm{R}_{\mathrm{f}}=0.2(\mathrm{DCM})$, chromatography eluent: $20 \%$ DCM in petroleum ether to DCM.

IR (Reflection): $\tilde{v}=3497,2954,2923,1693,1620,1420,1396,1325,1283,1164$, 1110, 1067, 1017, 986, 943, 847, 674; ${ }^{1} \mathbf{H}$ NMR (400 MHz, $\left.\mathrm{CDCl}_{3}\right): \delta=7.63(\mathrm{~d}, J=$ $8.1 \mathrm{~Hz}, 2 \mathrm{H}, 3 \mathbf{s a}), 7.57$ (d, $J=8.1 \mathrm{~Hz}, 2 \mathrm{H}, 3 \mathbf{s b}), 7.34$ (d, $J=8.1 \mathrm{~Hz}, 2 \mathrm{H}, 3 \mathbf{s b}), 7.31$ (d, $J=8.1 \mathrm{~Hz}, 2 \mathrm{H}, 3 \mathbf{s a}), 4.57(\mathrm{dd}, J=8.1,5.8 \mathrm{~Hz}, 1 \mathrm{H}, 3 \mathbf{s a}), 4.49$ (s, 2H, 3sb), 3.26 (t, $J=$ $7.0 \mathrm{~Hz}, 2 \mathrm{H}, 3 \mathbf{s b}$ ), 2.67 (s, 3H, 3sa), 2.38-2.60 (m, 3H, 3sa), 2.06-1.96 (m, 2H, 3sb), 1.91-1.77 (m, 1H, 3sa; 2H, 3sb); ${ }^{13} \mathbf{C}$ NMR (101 MHz, $\left.\mathrm{CDCl}_{3}\right): \delta=175.6$ (s), 175.2 (s), 145.5 (s), 140.9 (s), 130.5 (q, $J=32.7, \mathrm{~s}), 128.4$ (d), 126.8 (d), 126.2 (q, $J=3.8$, d), $125.8(\mathrm{q}, J=3.8, \mathrm{~d}), 124.0(\mathrm{q}, J=273.1, \mathrm{~s}), 64.3(\mathrm{~d}), 46.8(\mathrm{t}), 46.3(\mathrm{t}), 30.8(\mathrm{t})$, 30.0 (t), 28.4 (q), $28.4(\mathrm{t}), 17.9(\mathrm{t}) ;{ }^{19} \mathbf{F}$ NMR (283 MHz, $\left.\mathrm{CDCl}_{3}\right): \delta=-62.6,-62.6$;

HRMS (ESI): Calcd for $\mathrm{C}_{12} \mathrm{H}_{12} \mathrm{~F}_{3} \mathrm{NNaO}$ [M+Na]: 266.0763, found: 266.0761

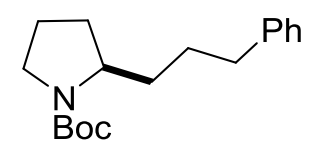

tert-Butyl-2-(3-phenylpropyl)pyrrolidine-1-carboxylate $( \pm)$

4a Colorless oil, $35.9 \mathrm{mg}, 62 \%$ yield.

$\mathrm{R}_{\mathrm{f}}=0.2(30 \% \mathrm{DCM} / \mathrm{PE})$, chromatography eluent: petroleum ether to $30 \% \mathrm{DCM}$ in petroleum ether.

IR (Reflection): $\tilde{v}=3062,3026,2971,2931,2870,1945,1877,1804,1691,1603$, 1496, 1478, 1454, 1395, 1365, 1253, 1172, 1102, 1030, 914, 877, 772, 748, 699; ${ }^{1} \mathbf{H}$ NMR (300 MHz, $\left.\mathrm{CDCl}_{3}\right): \delta=7.39-7.30(\mathrm{~m}, 2 \mathrm{H}), 7.29-7.20(\mathrm{~m}, 3 \mathrm{H}), 3.97-3.64(\mathrm{~m}$, $1 \mathrm{H}), 3.55-3.28(\mathrm{~m}, 2 \mathrm{H}), 2.84-2.57(\mathrm{~m}, 2 \mathrm{H}), 2.07-1.78(\mathrm{~m}, 4 \mathrm{H}), 1.78-1.60(\mathrm{~m}, 3 \mathrm{H})$, 1.56-1.39 (m, 10H); ${ }^{13} \mathbf{C}$ NMR (75 MHz, $\left.\mathrm{CDCl}_{3}\right): \delta=154.7$ (s), 142.5 (s), 128.5 (d), 128.4 (d), 125.8 (d), 126.7 (d), 126.6 (d), 125.0 (d), 120.9 (d), 79.0 (s), 57.3 (d), 46.5 $(\mathrm{t}), 46.2(\mathrm{t}), 36.1(\mathrm{t}), 34.1(\mathrm{t}), 34.1(\mathrm{t}), 30.8(\mathrm{t}), 30.0(\mathrm{t}), 28.6(\mathrm{q}), 28.2(\mathrm{t}), 23.8(\mathrm{t}), 23.2$ (t); HRMS (ESI): Calcd for $\mathrm{C}_{18} \mathrm{H}_{27} \mathrm{NNaO}_{2}\left[\mathrm{M}^{\bullet+}\right]$ : 312.1934 , found: 312.1931 . 


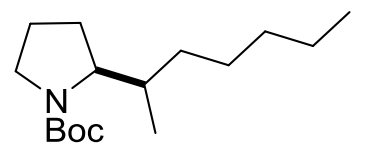

tert-Butyl-2-(heptan-2-yl)pyrrolidine-1-carboxylate $( \pm)$

4b Colorless oil, $21.5 \mathrm{mg}, 40 \%$ yield.

$\mathrm{R}_{\mathrm{f}}=0.2(30 \% \mathrm{DCM} / \mathrm{PE})$, chromatography eluent: petroleum ether to $30 \% \mathrm{DCM}$ in petroleum ether.

IR (Reflection): $\tilde{v}=2963,2929,2873,1698,1456,1397,1366,1254,1173,1107$, 916, 875, 772; ${ }^{1} \mathbf{H}$ NMR $\left(300 \mathrm{MHz}, \mathrm{CDCl}_{3}\right): \delta=3.72-3.47(\mathrm{~m}, 1.5 \mathrm{H}), 3.34-3.15(\mathrm{~m}$, 1.5H), 2.26-1.61 (m, 6H), 1.57-1.40 (m, 10H), 1.38-1.03 (m, 7H), 0.93-0.70 (m, 5H); ${ }^{13}$ C NMR (75 MHz, $\mathrm{CDCl}_{3}$ ): $\delta=155.1$ (s), 154.8 (s), 79.0 (s), 62.4 (d), 61.1 (d), 47.4 $(\mathrm{t}), 47.1(\mathrm{t}), 46.1(\mathrm{t}), 45.8(\mathrm{t}), 34.2(\mathrm{t}), 32.3(\mathrm{t}), 31.2(\mathrm{t}), 28.7(\mathrm{~d}), 27.4(\mathrm{t}), 22.8(\mathrm{t}), 16.7$ (q), 14.2 (q); HRMS (ESI): Calcd for $\mathrm{C}_{16} \mathrm{H}_{31} \mathrm{NNaO}_{2}$ [M+Na]: 292.2247, found: 292.2250 .

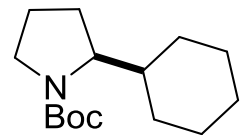

tert-Butyl-2-cyclohexylpyrrolidine-1-carboxylate $( \pm)$

4c Colorless oil, $30.9 \mathrm{mg}, 61 \%$ yield.

$\mathrm{R}_{\mathrm{f}}=0.2(30 \% \mathrm{DCM} / \mathrm{PE})$, chromatography eluent: petroleum ether to $30 \% \mathrm{DCM}$ in petroleum ether.

IR (Reflection): $\tilde{v}=2973,2928,2853,1694,1478,1450,1390,1365,1253,1167$, 1108, 772; ${ }^{1} \mathbf{H}$ NMR (300 MHz, $\left.\mathrm{CDCl}_{3}\right): \delta=3.77-3.28(\mathrm{~m}, 2 \mathrm{H}), 3.25-3.14(\mathrm{~m}, 1 \mathrm{H})$, 
1.84-1.53 (m, 10H), $1.45(\mathrm{~s}, 9 \mathrm{H}), 1.29-0.83(\mathrm{~m}, 6 \mathrm{H}) ;{ }^{13} \mathrm{C} \mathrm{NMR}\left(75 \mathrm{MHz}, \mathrm{CDCl}_{3}\right): \delta=$ $155.3(\mathrm{~s}), 79.1(\mathrm{~s}), 62.0(\mathrm{~d}), 47.1(\mathrm{t}), 46.5(\mathrm{t}), 41.8(\mathrm{t}), 40.9(\mathrm{t}), 30.3(\mathrm{t}), 28.7(\mathrm{q}), 28.1$ (t), 26.8 (t), 26.5 (t); HRMS (ESI): Calcd for $\mathrm{C}_{15} \mathrm{H}_{27} \mathrm{NNaO}_{2}$ [M+Na]: 276.1934, found: 276.1931 .

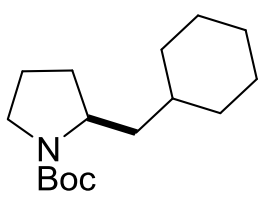

tert-Butyl-2-(cyclohexylmethyl)pyrrolidine-1-carboxylate $( \pm)$

4d Colorless oil, $20.3 \mathrm{mg}, 38 \%$ yield.

$\mathrm{R}_{\mathrm{f}}=0.2(30 \% \mathrm{DCM} / \mathrm{PE})$, chromatography eluent: petroleum ether to $30 \% \mathrm{DCM}$ in petroleum ether.

IR (Reflection): $\tilde{v}=2972,2924,2852,1696,1478,1449,1392,1365,1254,1171$, 1104, 874, 771; ${ }^{1} \mathbf{H}$ NMR (300 MHz, $\mathrm{CDCl}_{3}$ ): $\delta=3.82$ (br. s, 1H), 3.30 (br. s, 2H), 1.93-1.73 (m, 4H), 1.73-1.55 (m, 6H), $1.45(\mathrm{~s}, 9 \mathrm{H}), 1.25-1.05(\mathrm{~m}, 5 \mathrm{H}), 0.97-0.83(\mathrm{~m}$, 2H); ${ }^{13}$ C NMR (75 MHz, $\left.\mathrm{CDCl}_{3}\right): \delta=154.7$ (s), 79.0 (s), 55.3 (d), 46.4 (t), 45.9 (t), $42.6(\mathrm{t}), 41.8(\mathrm{t}), 35.5(\mathrm{~d}), 34.6(\mathrm{t}), 32.9(\mathrm{t}), 31.0(\mathrm{t}), 28.7(\mathrm{q}), 26.7(\mathrm{t}), 26.6(\mathrm{t}), 26.4(\mathrm{t})$, 24.0 (t), 23.0 (t); HRMS (ESI): Calcd for $\mathrm{C}_{16} \mathrm{H}_{29} \mathrm{NNaO}_{2}$ [M+Na]: 290.2090, found: 290.2093.

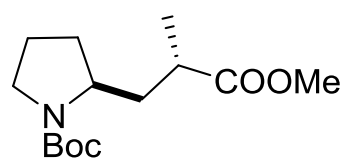

tert-Butyl-2-((S)-3-methoxy-2-methyl-3-oxopropyl)pyrrolidine-1-carboxylate $( \pm)$

4e Colorless oil, $34.2 \mathrm{mg}, 63 \%$ yield.

$\mathrm{R}_{\mathrm{f}}=0.2(30 \% \mathrm{DCM} / \mathrm{PE})$, chromatography eluent: petroleum ether to $30 \% \mathrm{DCM}$ in 
petroleum ether.

IR (Reflection): $\tilde{v}=2975,2878,1737,1694,1479,1455,1392,1366,1253,1165$, 1106, 863, 773; ${ }^{1} \mathbf{H}$ NMR $\left(300 \mathrm{MHz}, \mathrm{CDCl}_{3}\right): \delta=4.01-3.55(\mathrm{~m}, 4 \mathrm{H}), 3.46-3.16(\mathrm{~m}$, 2H), 2.59-2.33 (m, 1H), 2.17-1.72 (m, 4H), 1.68-1.58 (m, 2H), $1.46(\mathrm{~s}, 9 \mathrm{H}), 1.17$ (d, $J$ $=7.0 \mathrm{~Hz}, 3 \mathrm{H}) ;{ }^{13} \mathrm{C}$ NMR $\left(75 \mathrm{MHz}, \mathrm{CDCl}_{3}\right): \delta=176.8$ (s), 154.8 (s), 154.6 (s), 79.3 (s), $79.0(\mathrm{~s}), 55.7(\mathrm{~d}), 55.4(\mathrm{~d}), 51.6(\mathrm{t}), 46.4(\mathrm{t}), 45.8(\mathrm{t}), 39.0(\mathrm{t}), 38.4(\mathrm{t}), 37.0(\mathrm{q})$, 28.5 (q); HRMS (ESI): Calcd for $\mathrm{C}_{28} \mathrm{H}_{50} \mathrm{~N}_{2} \mathrm{NaO}_{8}$ [2M+Na]: 565.3459, found: 565.3460.

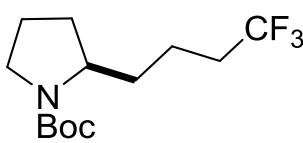

tert-Butyl-2-(4,4,4-trifluorobutyl)pyrrolidine-1-carboxylate $( \pm)$

4f Colorless oil, $42.7 \mathrm{mg}, 76 \%$ yield.

$\mathrm{R}_{\mathrm{f}}=0.4(50 \% \mathrm{DCM} / \mathrm{PE})$, chromatography eluent: $10 \%$ to $50 \%$ DCM in petroleum.

IR (Reflection): $\tilde{v}=2974,2878,1697,1479,1458,1395,1367,1346,1253,1221$, $1171,1141,1112,1058,1007,915,878,773,657 ;{ }^{1} \mathbf{H}$ NMR $\left(400 \mathrm{MHz}, \mathrm{CDCl}_{3}\right): \delta=$ 3.75 (br. s, 1H), 3.45-3.22 (m, 2H), 2.20-2.01 (m, 2H), 1.99-1.75 (m, 4H), 1.71-1.11 $(\mathrm{m}, 13 \mathrm{H}) ;{ }^{13} \mathbf{C}$ NMR (101 MHz, $\left.\mathrm{CDCl}_{3}\right): \delta=154.8$ (s), 127.3 (q, $\left.J=276.3 \mathrm{~Hz}, \mathrm{~s}\right)$, 79.3 (s), 56.9 (d), 46.5 (d, $J=38.4 \mathrm{~Hz}, \mathrm{t}), 33.9$ (q, $J=28.4 \mathrm{~Hz}, \mathrm{t}), 30.5$ (d, $J=89.4 \mathrm{~Hz}$ t), $28.7(\mathrm{q}), 23.6(\mathrm{~d}, J=81.3 \mathrm{~Hz}, \mathrm{t}), 19.0(\mathrm{t}) ;{ }^{19} \mathbf{F} \mathbf{N M R}\left(283 \mathrm{MHz}, \mathrm{CDCl}_{3}\right): \delta=-66.5$, -66.5; HRMS (ESI): Calcd for $\mathrm{C}_{13} \mathrm{H}_{22} \mathrm{~F}_{3} \mathrm{NNaO}_{2}$ [M+Na]: 304.1495, found: 304.1494.

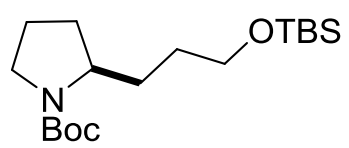

tert-Butyl-2-(3-((tert-butyldimethylsilyl)oxy)propyl)pyrrolidine-1-carboxylate $( \pm)$ 
4g Colorless oil, $46.1 \mathrm{mg}, 67 \%$ yield.

$\mathrm{R}_{\mathrm{f}}=0.2(\mathrm{DCM})$, chromatography eluent: $20 \%$ DCM in petroleum ether to DCM.

IR (Reflection): $\tilde{v}=2956,2930,2859,1697,1473,1461,1391,1365,1253,1171$, 1099, 939, 835, 774; ${ }^{1} \mathbf{H}$ NMR (300 MHz, $\left.\mathrm{CDCl}_{3}\right): \delta=3.85-3.50(\mathrm{~m}, 3 \mathrm{H}), 3.45-3.19$ (m, 2H), 1.95-1.60 (m, 5H), 1.52-1.21 (m, 12H), 0.88 (s, 9H), $0.04(\mathrm{~s}, 6 \mathrm{H}) ;{ }^{13} \mathbf{C}$ NMR $\left(75 \mathrm{MHz}, \mathrm{CDCl}_{3}\right): \delta=154.7(\mathrm{~s}), 147.7(\mathrm{~s}), 78.9(\mathrm{~s}), 63.3(\mathrm{~d}), 57.2(\mathrm{t}), 46.5(\mathrm{t}), 46.1(\mathrm{t})$, $31.3(\mathrm{t}), 30.8(\mathrm{t}), 29.8(\mathrm{t}), 28.6(\mathrm{q}), 26.0(\mathrm{q}), 23.7(\mathrm{t}), 23.2(\mathrm{t}), 18.4(\mathrm{t}),-5.23(\mathrm{q})$;

HRMS (ESI): Calcd for $\mathrm{C}_{18} \mathrm{H}_{37} \mathrm{NNaO}_{3} \mathrm{Si}$ [M+Na]: 366.2435, found: 366.2434.

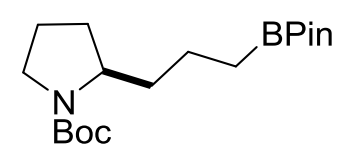

tert-Butyl-2-(3-(4,4,5,5-tetramethyl-1,3,2-dioxaborolan-2-yl)propyl)pyrrolidine-1-car boxylate $( \pm)$

4h Colorless oil, $40.7 \mathrm{mg}, 60 \%$ yield.

$\mathrm{R}_{\mathrm{f}}=0.4$ (30\% DCM/PE), chromatography eluent: petroleum ether to $30 \% \mathrm{DCM}$ in petroleum ether.

IR (Reflection): $\tilde{v}=3479,2976,2933,2880,1787,1754,1694,1479,1456,1395$, 1367, 1317, 1254, 1168, 1111, 1019, 968, 913, 847, 775, 702, 674; ${ }^{1}$ H NMR (300 $\left.\mathrm{MHz}, \mathrm{CDCl}_{3}\right): \delta=3.78-3.64(\mathrm{~m}, 2 \mathrm{H}), 3.37-3.21(\mathrm{~m}, 2 \mathrm{H}), 2.50(\mathrm{t}, J=8.1 \mathrm{~Hz}, 1 \mathrm{H})$, 2.04-1.63 (m, 8H), 1.48-1.42 (m, 12H), $1.23(\mathrm{~s}, 9 \mathrm{H}) ;{ }^{13} \mathrm{C} \mathrm{NMR}\left(75 \mathrm{MHz}, \mathrm{CDCl}_{3}\right): \delta=$ $174.4(\mathrm{~s}), 83.0(\mathrm{~s}), 83.0(\mathrm{~s}), 57.3(\mathrm{~d}), 46.6(\mathrm{t}), 46.1(\mathrm{t}), 33.1(\mathrm{t}), 28.7(\mathrm{q}), 28.6(\mathrm{q}), 28.2$ (q), 28.2 (q), 25.0 (q), 24.9 (q), 21.0 (t), 17.6 (t), 15.6 (q); HRMS (ESI): Calcd for $\mathrm{C}_{18} \mathrm{H}_{34} \mathrm{BNNaO}_{4}$ [M+Na]: 362.2473, found: 362.2474. 
<smiles>c1ccc(C2CCCS2)cc1</smiles>

2-phenyltetrahydrothiophene

6a Colorless oil, $19.7 \mathrm{mg}, 60 \%$ yield.

$\mathrm{R}_{\mathrm{f}}=0.4(30 \% \mathrm{DCM} / \mathrm{PE})$, chromatography eluent: petroleum ether to $10 \% \mathrm{DCM}$ in petroleum ether.

${ }^{1} \mathbf{H}$ NMR (300 MHz, $\left.\mathrm{CDCl}_{3}\right): \delta=7.45-7.39(\mathrm{~m}, 2 \mathrm{H})$, 7.35-7.27 (m, 2H), 7.26-7.19 (m, $1 \mathrm{H}), 4.53(\mathrm{t}, J=4.53 \mathrm{~Hz}, 1 \mathrm{H}), 3.21-3.10(\mathrm{~m}, 1 \mathrm{H}), 3.06-2.97(\mathrm{~m}, 1 \mathrm{H}), 2.44-2.24(\mathrm{~m}$, 2H), 2.08-1.89 (m, 2H). ${ }^{13} \mathbf{C}$ NMR (75 MHz, $\left.\mathrm{CDCl}_{3}\right): \delta=143.1$ (s), 128.5 (d), 127.8 (d), 127.1 (d), 52.9 (d), 40.7 (t), 33.7 (t), 31.2 (t); HRMS (EI): Calcd for $\mathrm{C}_{10} \mathrm{H}_{12} \mathrm{~S}$ $\left[\mathrm{M}^{\cdot+}\right]$ : 164.06542 , found: 164.06479 .

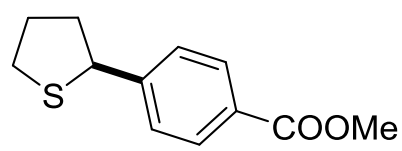

Methyl 4-(tetrahydrothiophen-2-yl)benzoate

6b Colorless oil, $35.0 \mathrm{mg}, 79 \%$ yield.

$\mathrm{R}_{\mathrm{f}}=0.3(30 \% \mathrm{DCM} / \mathrm{PE})$, chromatography eluent: petroleum ether to $10 \% \mathrm{DCM}$ in petroleum ether.

IR (Reflection): $\tilde{v}=2949,2860,1722,1610,1573,1436,1415,1279,1178,1110$, 1018, 966, 861, 806, 771, 709; ${ }^{1} \mathbf{H}$ NMR $\left(400 \mathrm{MHz}, \mathrm{CDCl}_{3}\right): \delta=7.89(\mathrm{~d}, J=8.4 \mathrm{~Hz}$, 2H), $7.41(\mathrm{~d}, J=8.3 \mathrm{~Hz}, 2 \mathrm{H}), 4.47(\mathrm{dd}, J=8.3,6.3 \mathrm{~Hz}, 1 \mathrm{H}), 3.83(\mathrm{~s}, 3 \mathrm{H}), 3.14-3.04$ (m, 1H), 3.00-2.90 (m, 1H), 2.39-2.29 (m, 1H), 2.24-2.14 (m, 1H), 2.01-1.80 (m, 2H). ${ }^{13}$ C NMR (101 MHz, $\mathrm{CDCl}_{3}$ ): $\delta$ = 167.0 (s), 148.7 (s), 129.9 (d), 129.0 (s), 127.8 (d), 
52.5 (d), 52.2 (q), 40.6 (t), 33.7 (t), 31.2 (t); HRMS (EI): Calcd for $\mathrm{C}_{12} \mathrm{H}_{14} \mathrm{O}_{2} \mathrm{~S}\left[\mathrm{M}^{\bullet+}\right]$ : 222.07090, found: 222.06975.

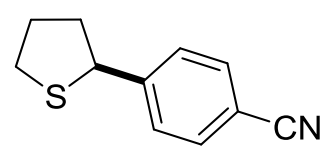

4-(tetrahydrothiophen-2-yl)benzonitrile

6c Colorless oil, $26.7 \mathrm{mg}, 71 \%$ yield.

$\mathrm{R}_{\mathrm{f}}=0.3(30 \% \mathrm{DCM} / \mathrm{PE})$, chromatography eluent: petroleum ether to $10 \% \mathrm{DCM}$ in petroleum ether.

${ }^{1} \mathbf{H}$ NMR $\left(300 \mathrm{MHz}, \mathrm{CDCl}_{3}\right): \delta=7.59(\mathrm{~d}, J=9.0 \mathrm{~Hz}, 2 \mathrm{H}), 7.52(\mathrm{~d}, J=9.0 \mathrm{~Hz}, 2 \mathrm{H})$, $4.53(\mathrm{t}, J=4.53 \mathrm{~Hz}, 1 \mathrm{H}), 3.21-3.12(\mathrm{~m}, 1 \mathrm{H}), 3.07-2.99(\mathrm{~m}, 1 \mathrm{H}), 2.47-2.36(\mathrm{~m}, 1 \mathrm{H})$, 2.32-2.21 (m, 1H), 2.10-1.80 (m, 2H). $\left.{ }^{13} \mathrm{C} \mathrm{NMR} \mathrm{(75} \mathrm{MHz,} \mathrm{CDCl}_{3}\right): \delta=149.2(\mathrm{~s})$, 132.5 (d), 128.7 (d), 119.1 (s), 111.0 (s), 52.5 (d), 40.7 (t), 33.9 (t), 31.3 (t); HRMS (EI): Calcd for $\mathrm{C}_{11} \mathrm{H}_{11} \mathrm{NS}\left[\mathrm{M}^{\bullet+}\right]$ : 189.06180 , found: 189.06067 .

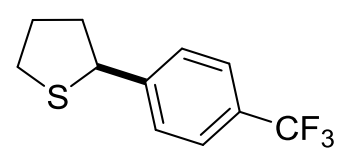

2-(4-(trifluoromethyl)phenyl)tetrahydrothiophene

6d Colorless oil, $30.1 \mathrm{mg}, 65 \%$ yield.

$\mathrm{Rf}=0.3(30 \% \mathrm{DCM} / \mathrm{PE})$, chromatography eluent: petroleum ether to $10 \% \mathrm{DCM}$ in petroleum ether.

${ }^{\mathbf{1}} \mathbf{H}$ NMR $(300 \mathrm{MHz}, \mathrm{CDCl} 3): \delta=7.55(\mathrm{~m}, 4 \mathrm{H}), 4.54(\mathrm{t}, \mathrm{J}=4.53 \mathrm{~Hz}, 1 \mathrm{H}), 3.21-3.12$ 
(m, 1H), 3.07-2.99 (m, 1H), 2.47-2.37 (m, 1H), 2.32-2.23 (m, 1H), 2.08-1.87 (m, 2H). ${ }^{13}$ C NMR (75 MHz, CDCl3): $\delta=147.5$ (s), 129.4 (q, $\left.J=32.4 \mathrm{~Hz}, \mathrm{~s}\right), 127.0$ (q, $J=$ $272.7 \mathrm{~Hz}, \mathrm{~s}), 125.5$ (q, $J=7.5 \mathrm{~Hz}, \mathrm{~d}), 52.3$ (d), 40.7 (t), 33.8 (t), 31.2 (t); ${ }^{19}$ F NMR (283 MHz, $\left.\mathrm{CDCl}_{3}\right): \delta=-62.45$; HRMS (EI): Calcd for $\mathrm{C}_{11} \mathrm{H}_{11} \mathrm{~F}_{3} \mathrm{~S}\left[\mathrm{M}^{\cdot+}\right]$ : 232.05281, found: 232.05372 .

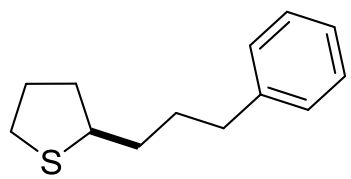

2-(3-phenylpropyl)Tetrahydrothiophene

6e Colorless oil, $30.1 \mathrm{mg}, 73 \%$ yield.

$\mathrm{R}_{\mathrm{f}}=0.6(30 \% \mathrm{DCM} / \mathrm{PE})$, chromatography eluent: petroleum ether to $10 \% \mathrm{DCM}$ in petroleum ether.

IR (Reflection): $\tilde{v}=3084,3061,3025,2933,2857,1943,1869,1802,1603,1495$, 1453, 1441, 1260, 1076, 1030, 960, 907, 748, 698; ${ }^{1} \mathbf{H}$ NMR (400 MHz, $\left.\mathrm{CDCl}_{3}\right): \delta=$ 7.31-7.24 (m, 2H), 7.22-7.14 (m, 3H), 3.41-3.28 (m, 1H), 2.93-2.78 (m, 2H), 2.68-2.59 (m, 2H), 2.14-2.01 (m, 2H), 1.95-1.49 (m, 7H); ${ }^{13} \mathrm{C}$ NMR (101 MHz, $\left.\mathrm{CDCl}_{3}\right): \delta=142.5(\mathrm{~s}), 128.5(\mathrm{~d}), 125.9(\mathrm{~d}), 49.4(\mathrm{~d}), 37.5(\mathrm{t}), 37.5(\mathrm{t}), 36.0(\mathrm{t}), 32.3(\mathrm{t})$, 31.0 (t), 30.5 (t); HRMS (EI): Calcd for $\mathrm{C}_{13} \mathrm{H}_{18} \mathrm{~S}\left[\mathrm{M}^{\cdot+}\right]$ : 206.11237, found: 206.11103 .

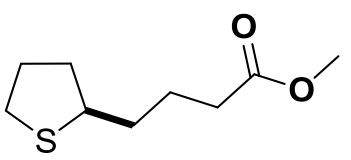

methyl -4-(tetrahydrothiophen-2-yl)butanoate

6f Colorless oil, $24.5 \mathrm{mg}, 65 \%$ yield.

$\mathrm{R}_{\mathrm{f}}=0.4(20 \% \mathrm{EA} / \mathrm{PE})$, chromatography eluent: petroleum ether to $20 \% \mathrm{EA}$ in petroleum ether. 
${ }^{1} \mathbf{H}$ NMR $\left(300 \mathrm{MHz}, \mathrm{CDCl}_{3}\right): \delta=3.67(\mathrm{~s}, 3 \mathrm{H}), 3.38-3.28(\mathrm{~m}, 1 \mathrm{H}), 2.92-2.80(\mathrm{~m}, 2 \mathrm{H})$, $2.32(\mathrm{t}, J=6.8 \mathrm{~Hz}, 2 \mathrm{H}), 2.15-1.99(\mathrm{~m}, 2 \mathrm{H}), 1.95-1.82(\mathrm{~m}, 1 \mathrm{H}), 1.76-1.47(\mathrm{~m}, 5 \mathrm{H}) ;{ }^{13} \mathrm{C}$ NMR (75 MHz, $\left.\mathrm{CDCl}_{3}\right): \delta=174.0$ (s), 51.7 (q), 48.9 (d), 37.4 (t), 37.3 (t), 34.0 (t), $32.3(\mathrm{t}), 30.5(\mathrm{t}), 24.5(\mathrm{t})$. HRMS (EI): Calcd for $\mathrm{C}_{9} \mathrm{H}_{16} \mathrm{O}_{2} \mathrm{~S}\left[\mathrm{M}^{+}\right]$: 188.08655, found: 188.08590.

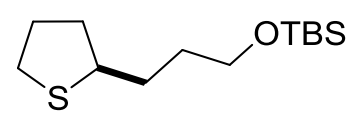

tert-butyldimethyl(3-(tetrahydrothiophen-2-yl)propoxy)silane

6g Colorless oil, $27.6 \mathrm{mg}, 53 \%$ yield.

$\mathrm{R}_{\mathrm{f}}=0.4(20 \% \mathrm{EA} / \mathrm{PE})$, chromatography eluent: petroleum ether to $20 \% \mathrm{EA}$ in petroleum ether.

${ }^{1} \mathbf{H}$ NMR (300 MHz, $\left.\mathrm{CDCl}_{3}\right): \delta=3.66-3.55(\mathrm{~m}, 2 \mathrm{H}), 3.42-3.28(\mathrm{~m}, 1 \mathrm{H}), 2.93-2.78(\mathrm{~m}$, 2H), 2.16-2.01 (m, 2H), 1.95-1.46 (m, 6H), 0.89 (s, 9H), $0.04(\mathrm{~s}, 6 \mathrm{H}) ;{ }^{13} \mathrm{C}$ NMR (75 $\left.\mathrm{MHz}, \mathrm{CDCl}_{3}\right): \delta=63.1(\mathrm{t}), 49.2(\mathrm{~d}), 37.5(\mathrm{t}), 34.1(\mathrm{t}), 32.5(\mathrm{t}), 32.3(\mathrm{t}), 30.5(\mathrm{t}), 26.1$ (q), 18.5 (s), -5.13 (q). HRMS (EI): Calcd for $\mathrm{C}_{12} \mathrm{H}_{25} \mathrm{OSiS}\left[\mathrm{M}^{+}-\mathrm{CH}_{3}\right]$ : 245.13899, found: 245.14045 .

\section{Reference}

[1] L. Guo, F. Song, S. Zhu, H. Li, L. Chu, Nat. Commun. 2018, 9, 4543.

[2] S. Murov, L., Handbook of Photochemistry, Marcel Dekker, New York, 1973. 

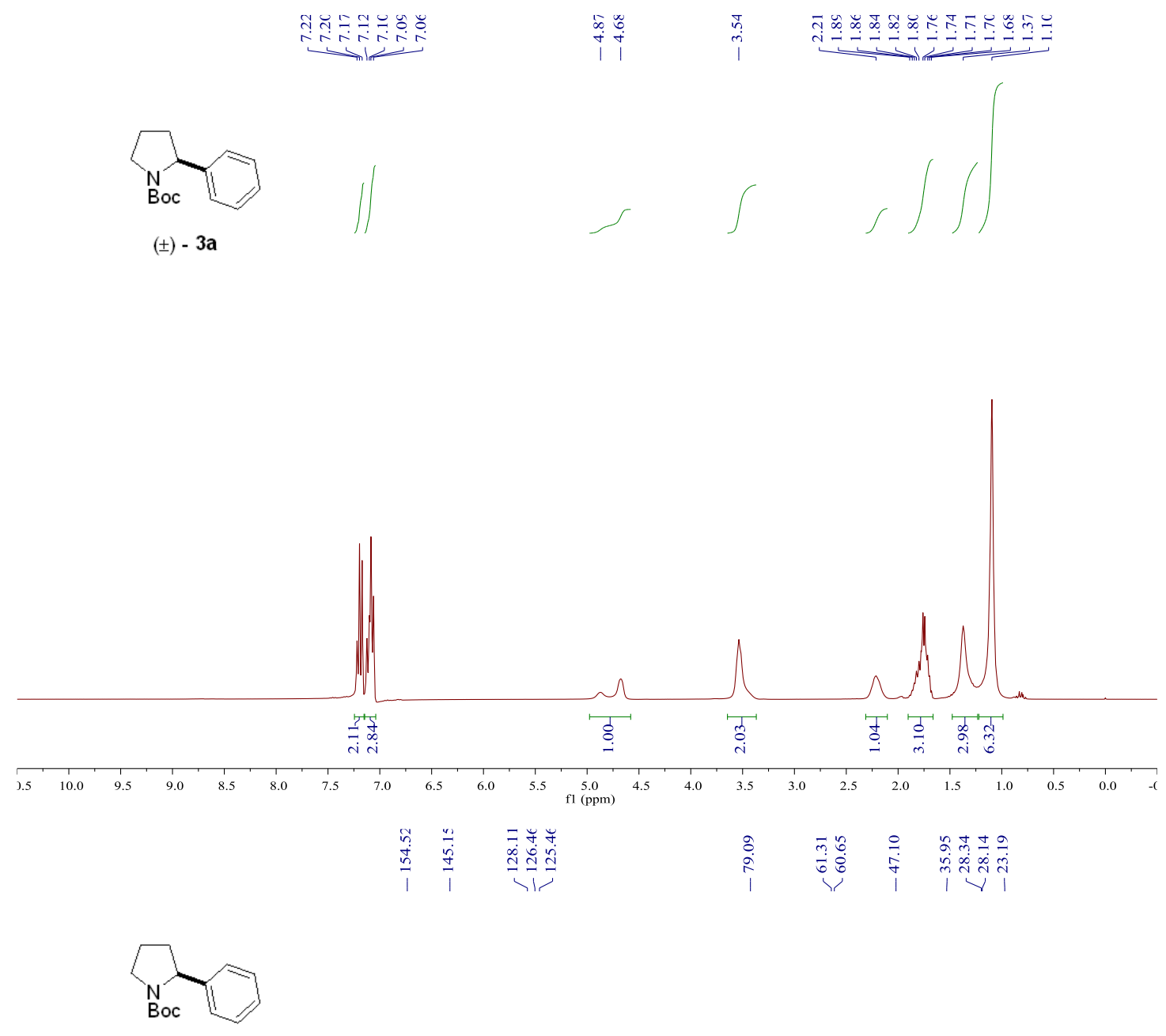

$( \pm)-3 a$

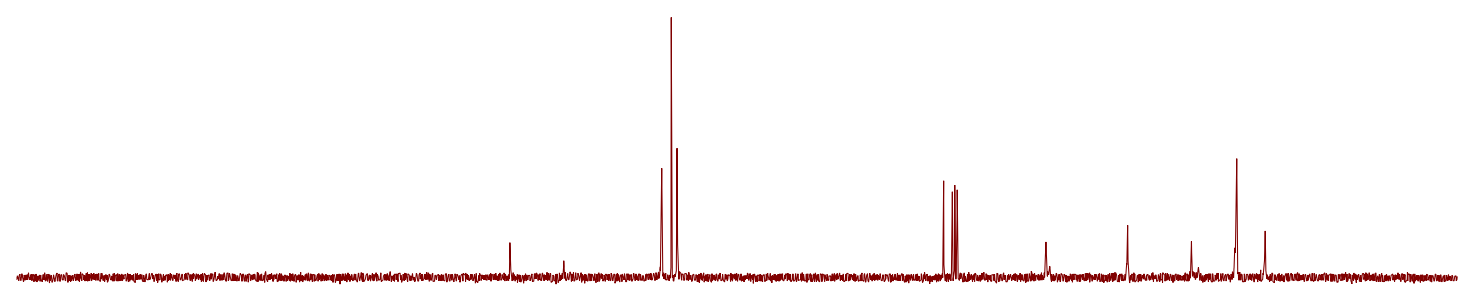

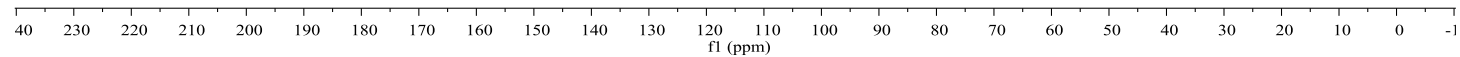




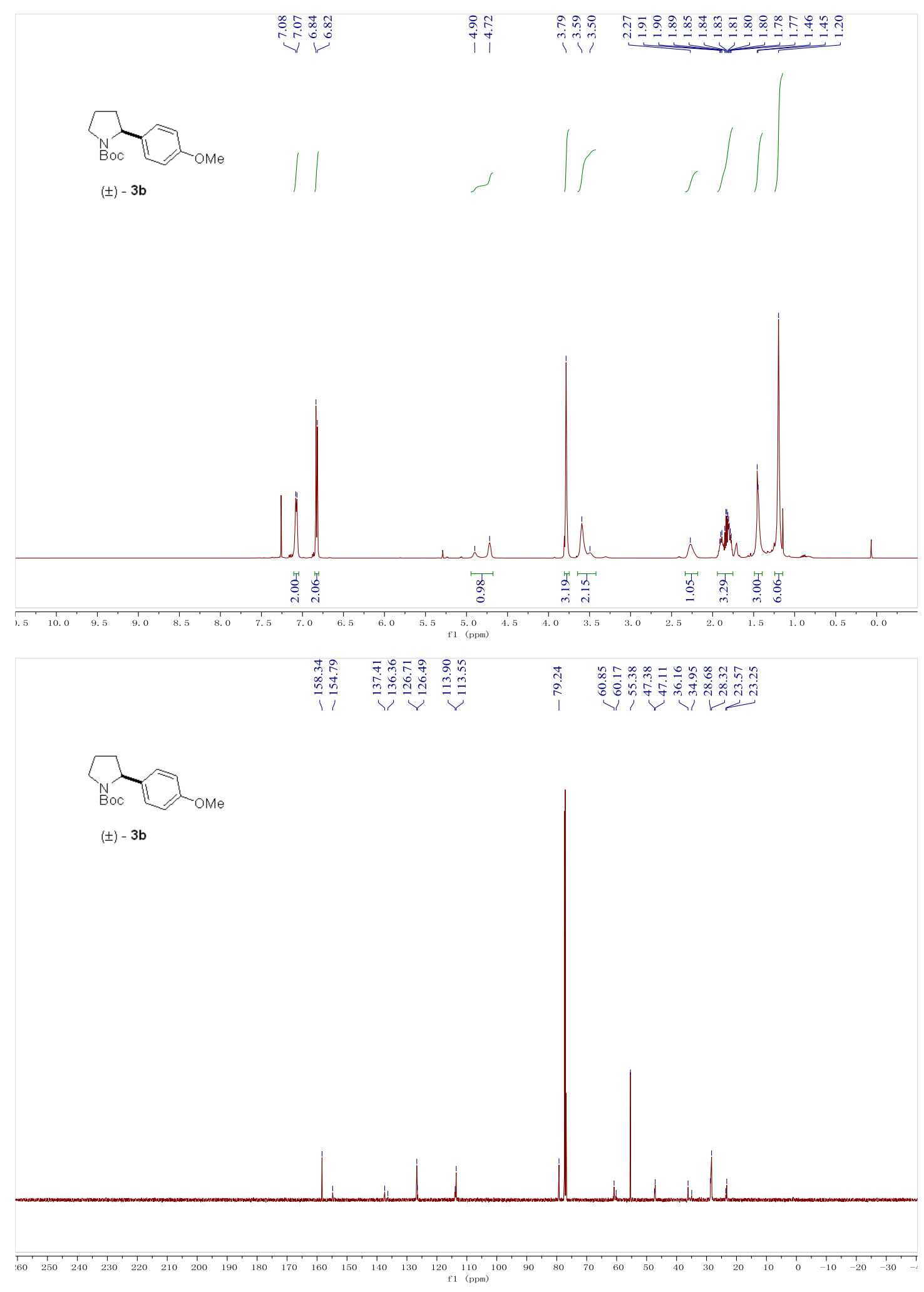




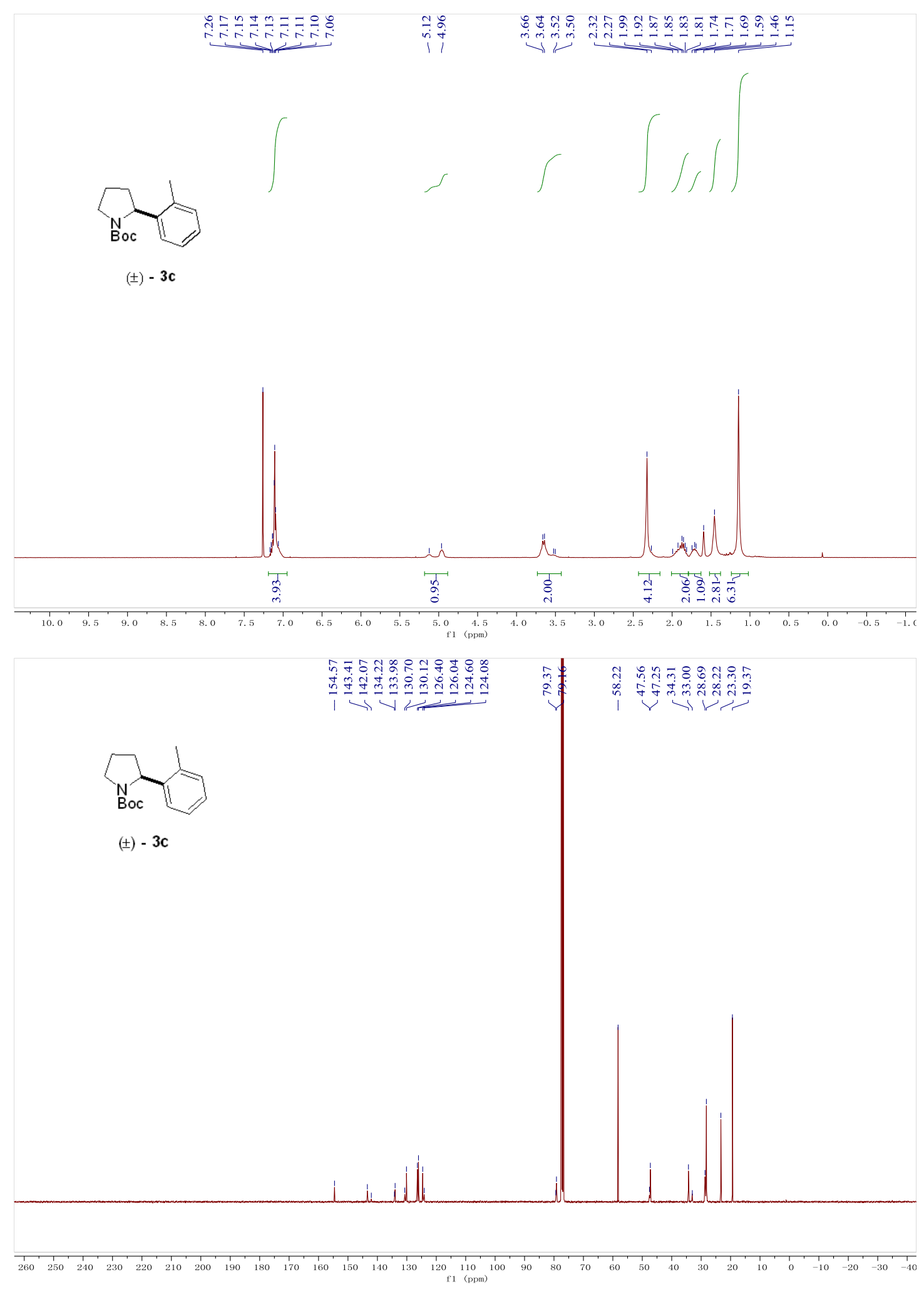




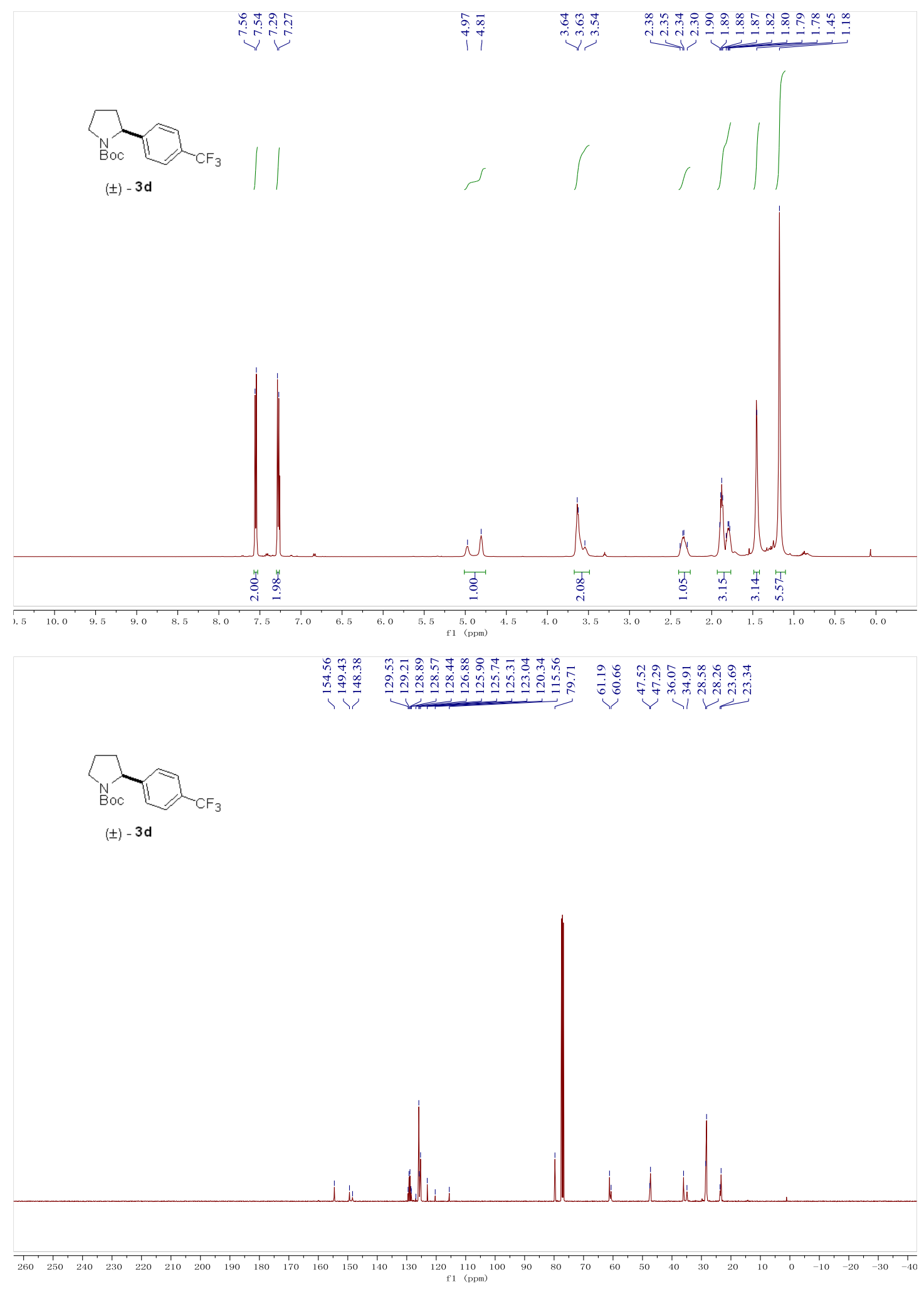




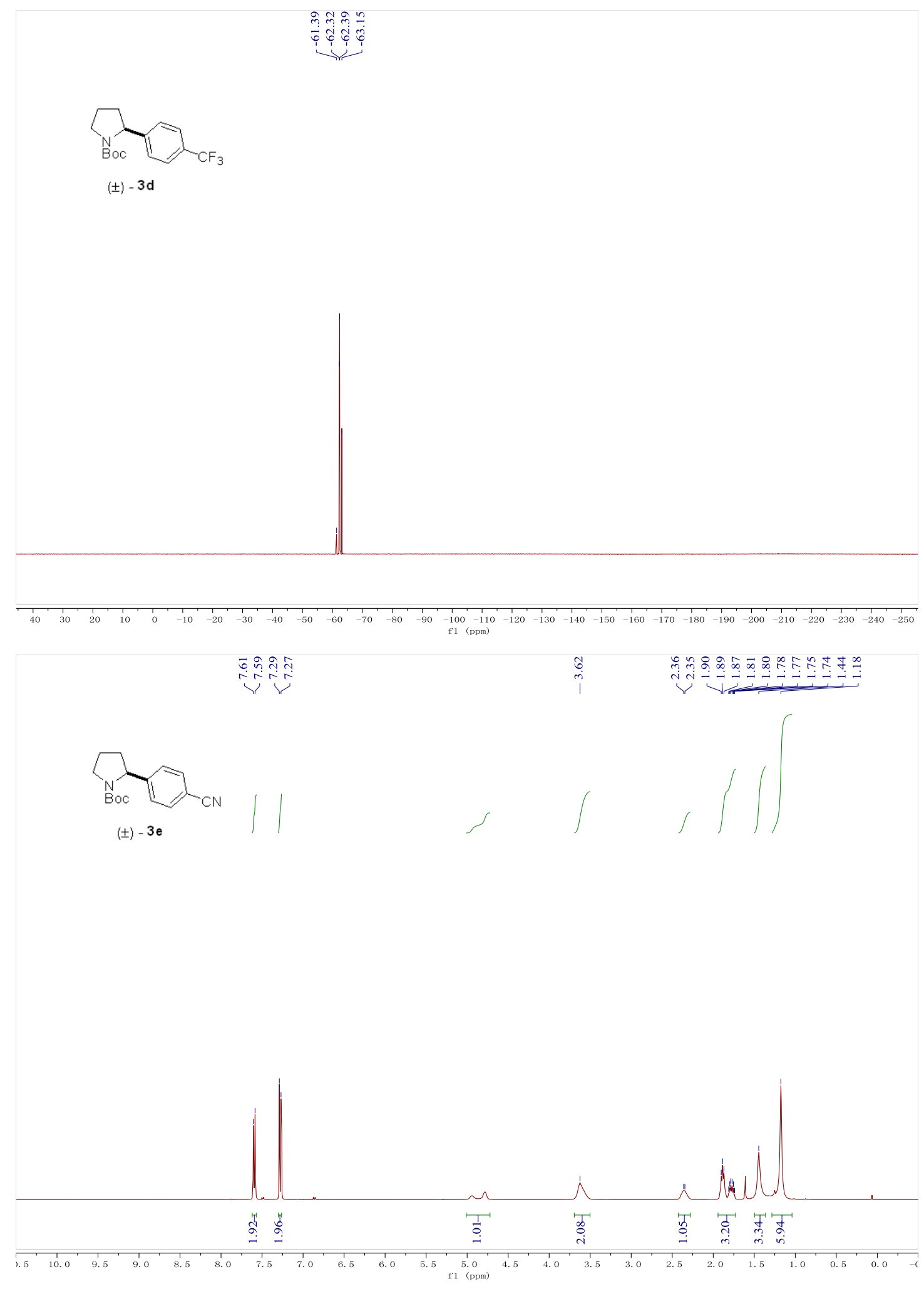




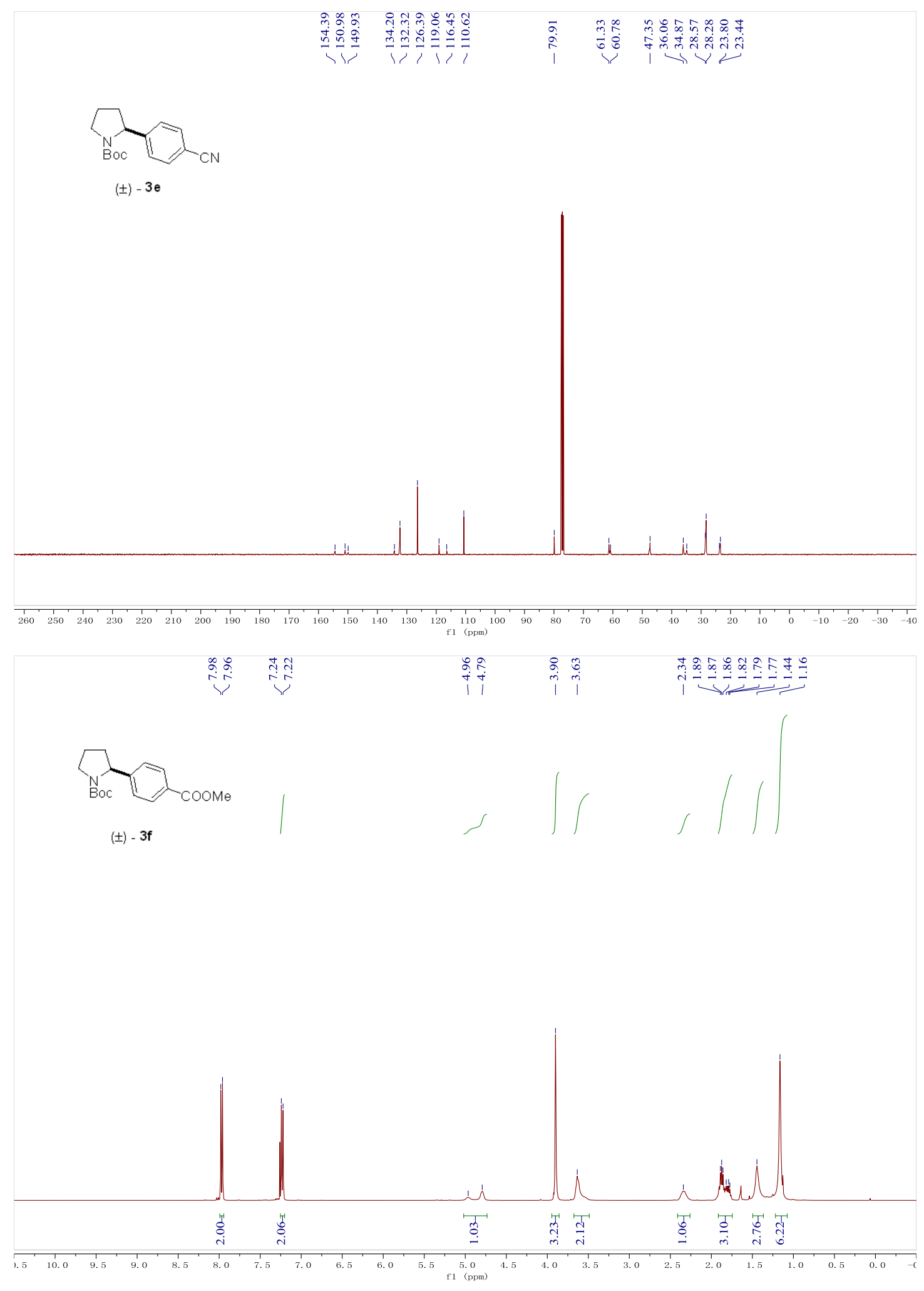




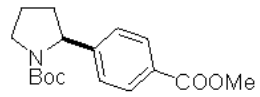

(士) - $3 f$

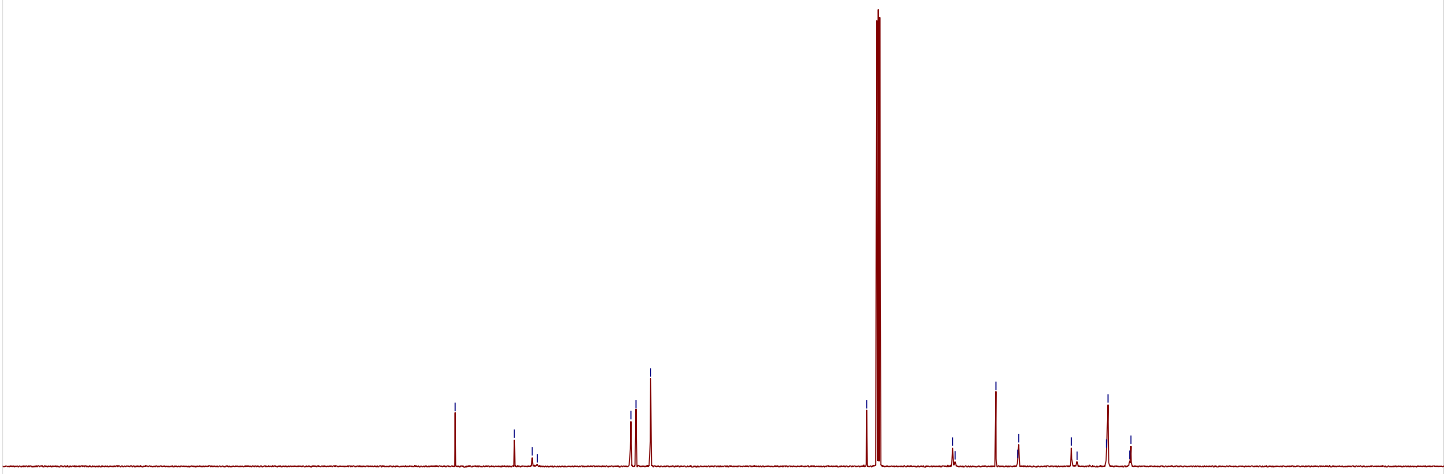

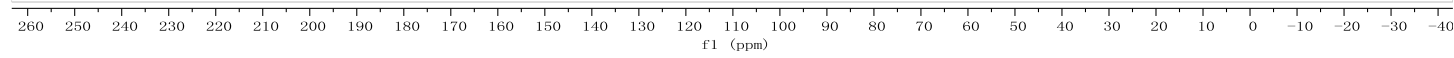

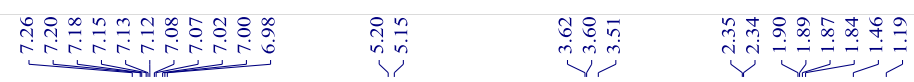<smiles>O=C(c1ccccc1)N1CCCC1c1ccccc1F</smiles>

$( \pm)-3 g$
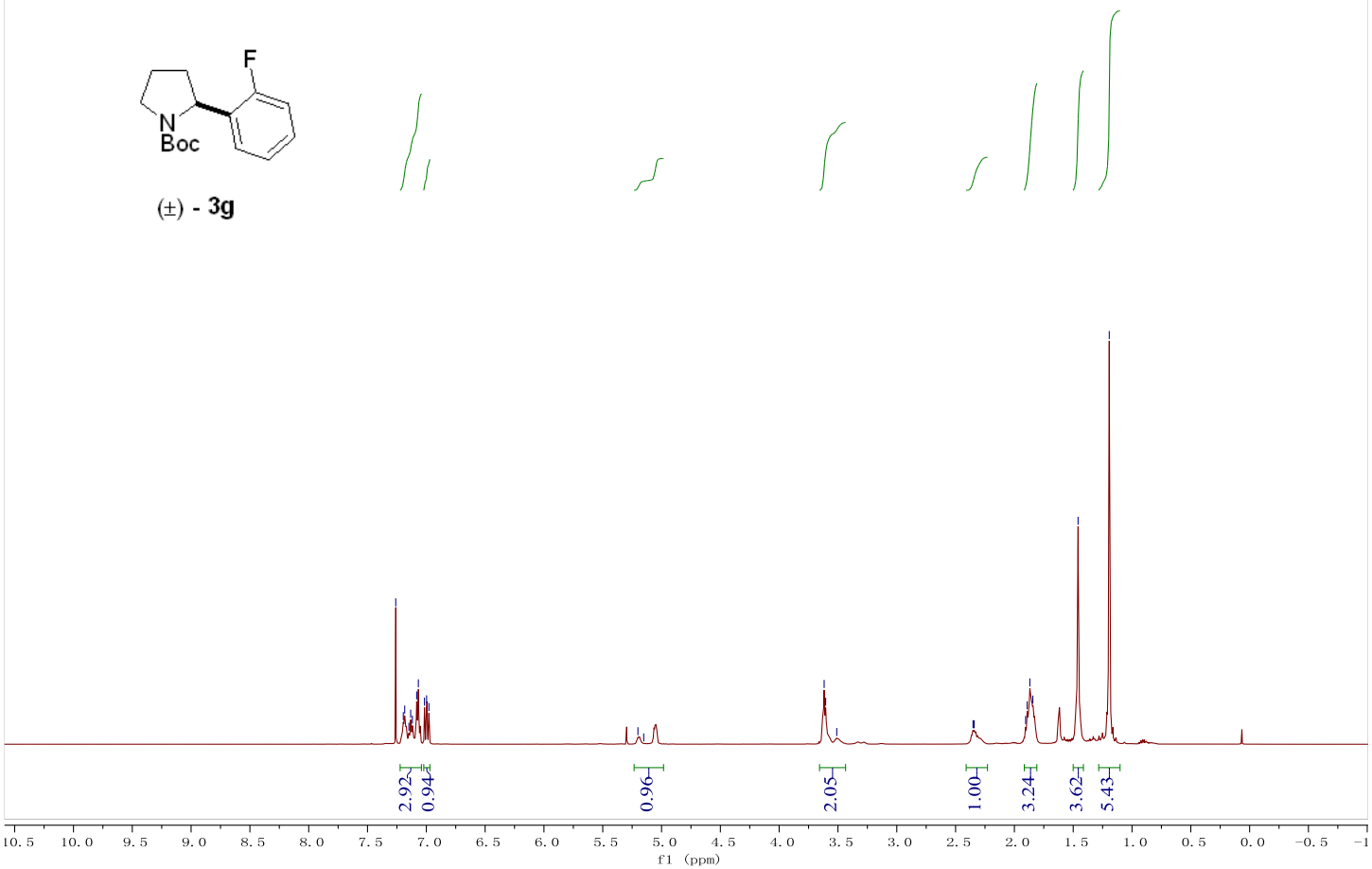


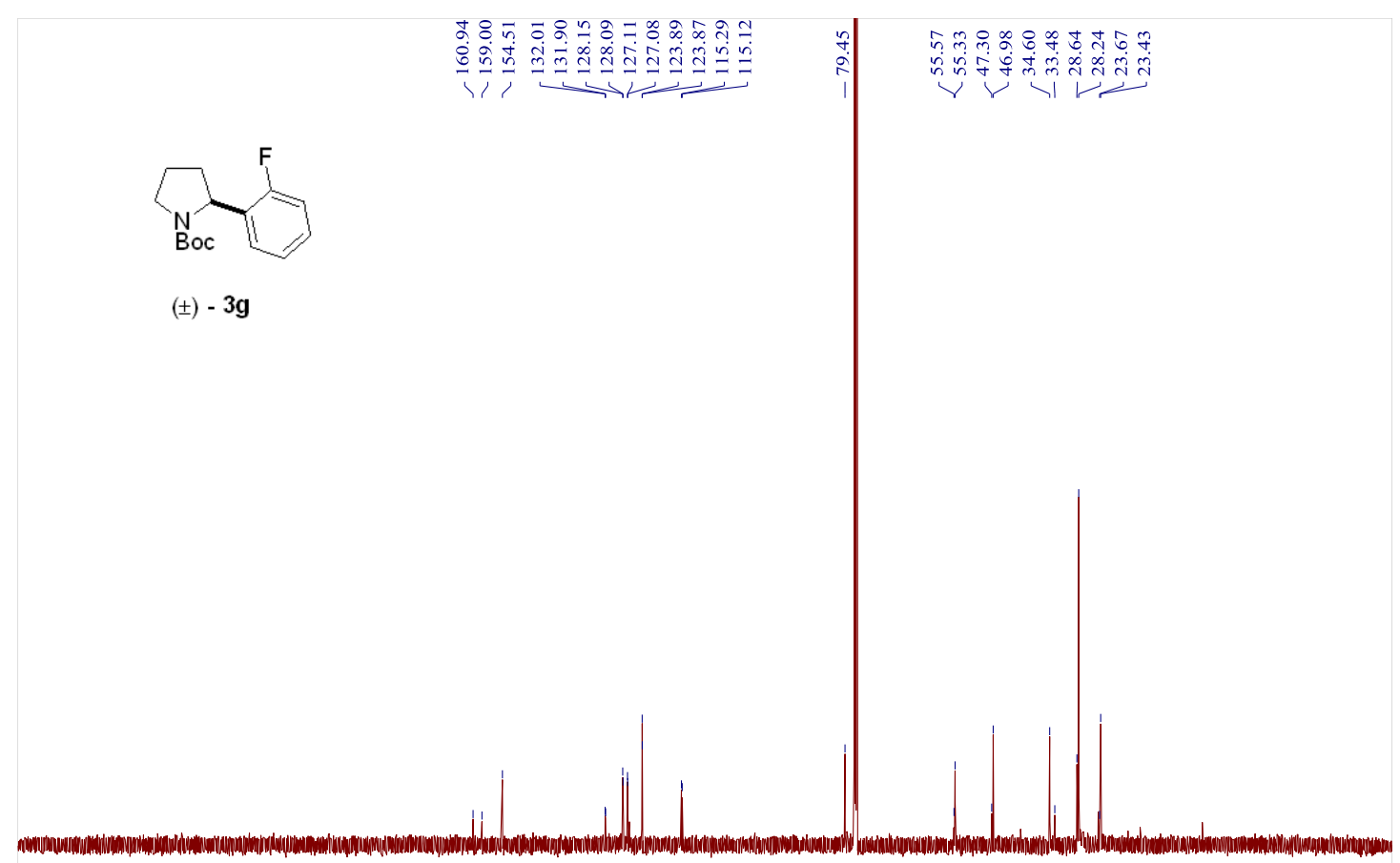

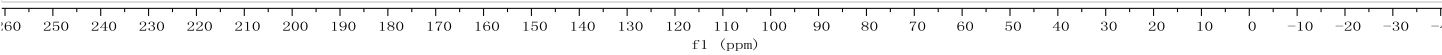
万人。

$\stackrel{\infty}{\circ} \stackrel{\infty}{\circ}=$

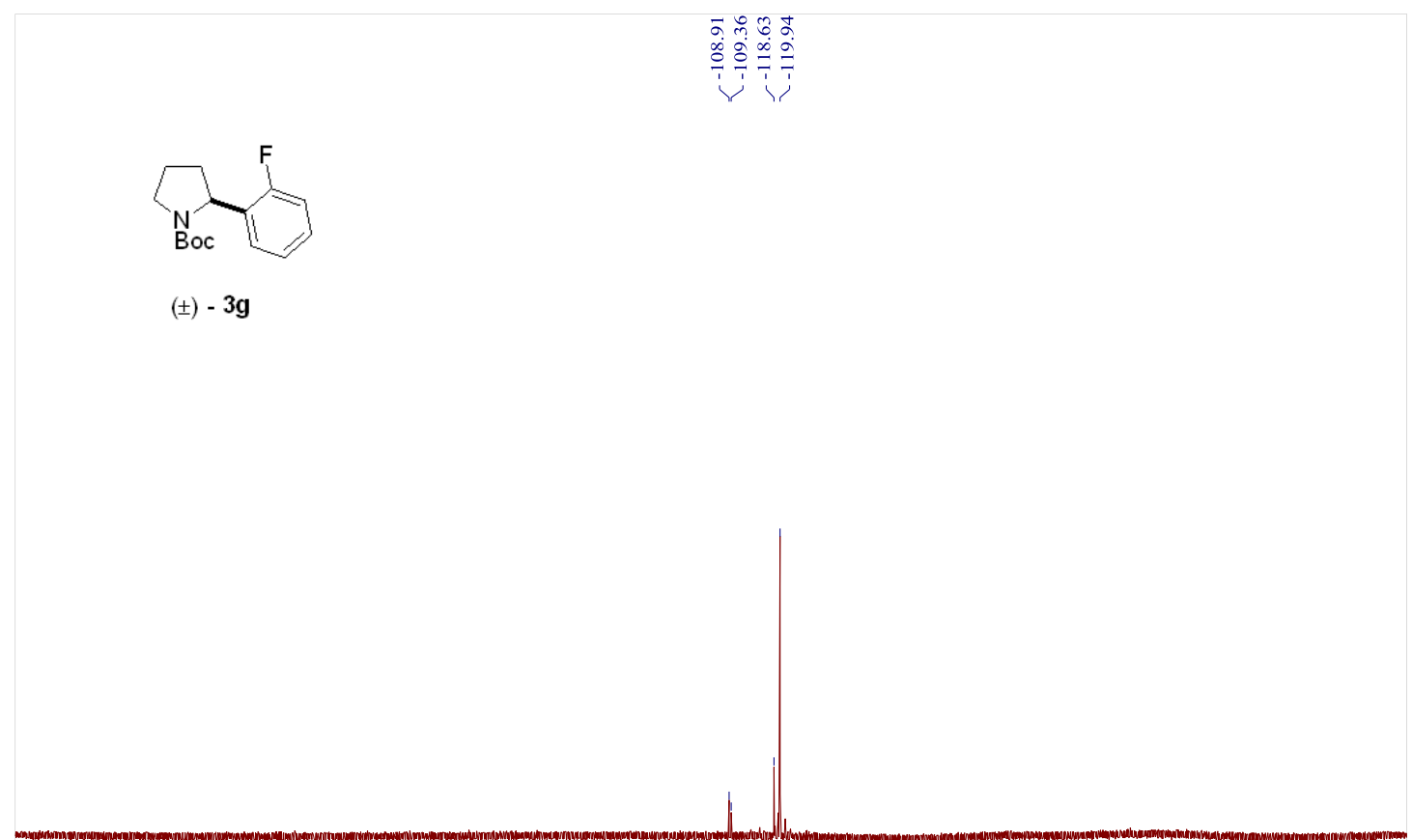

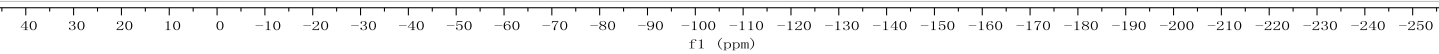




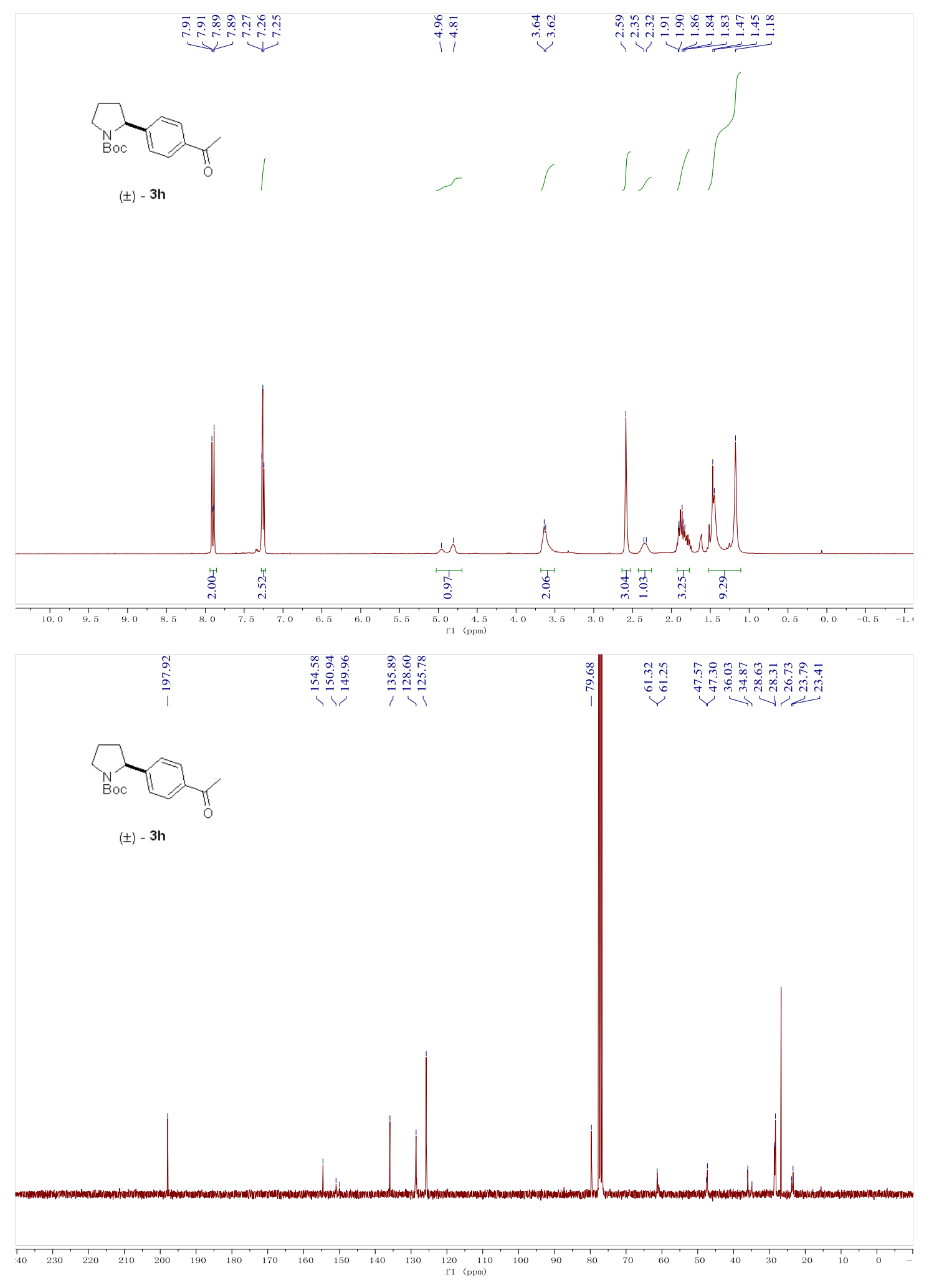




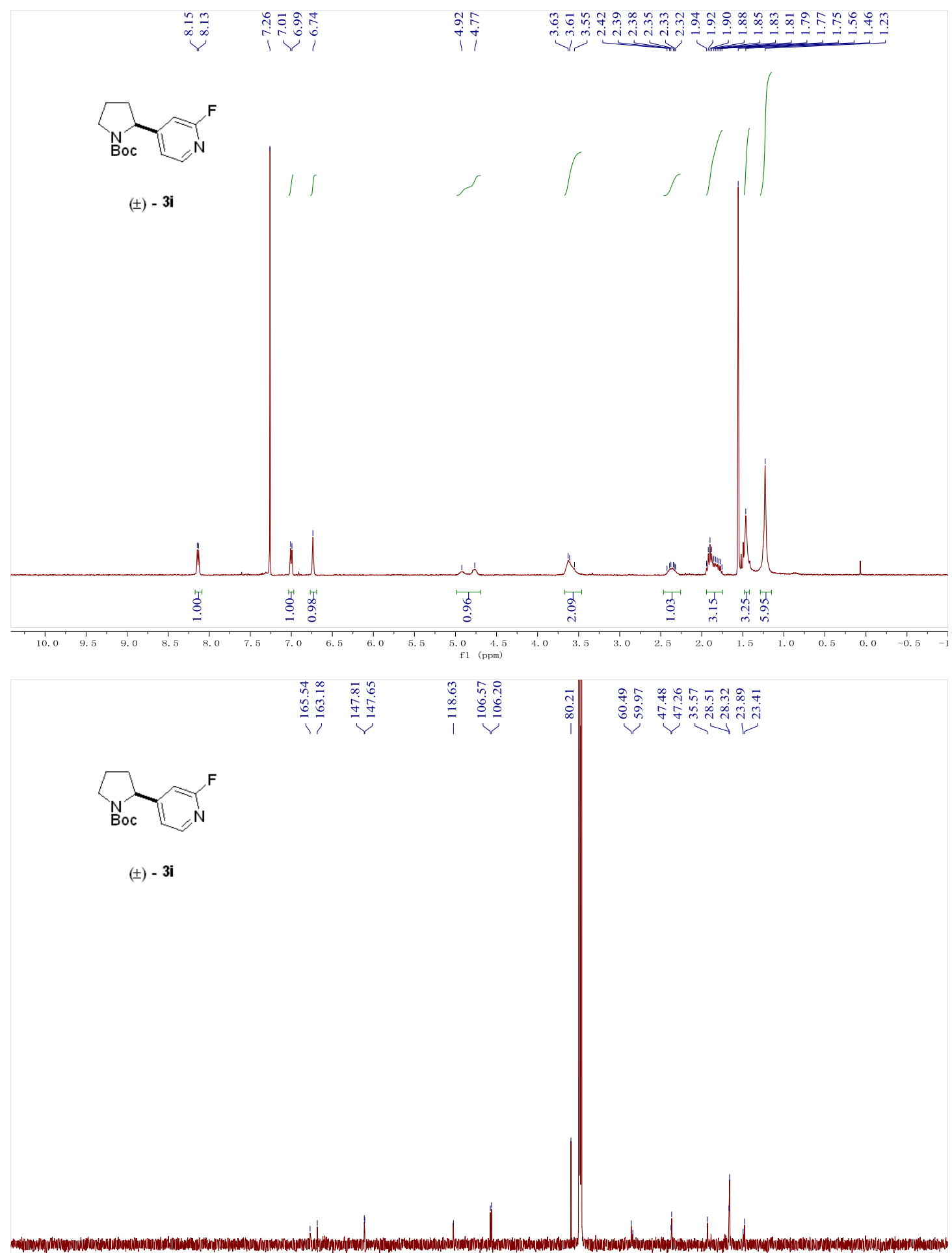

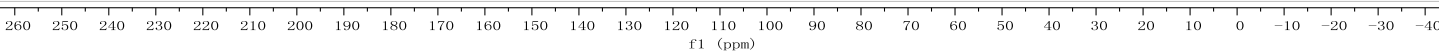




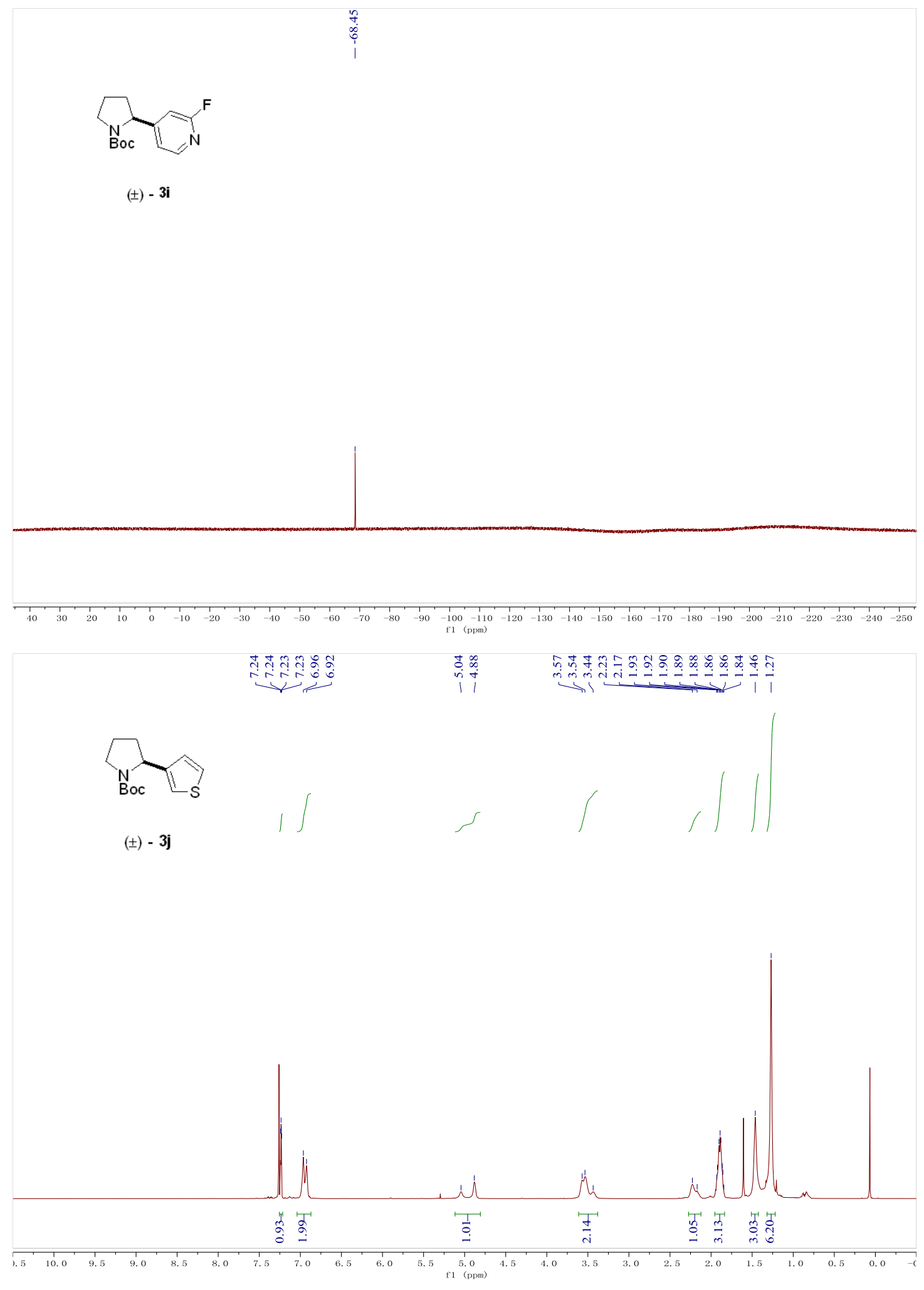




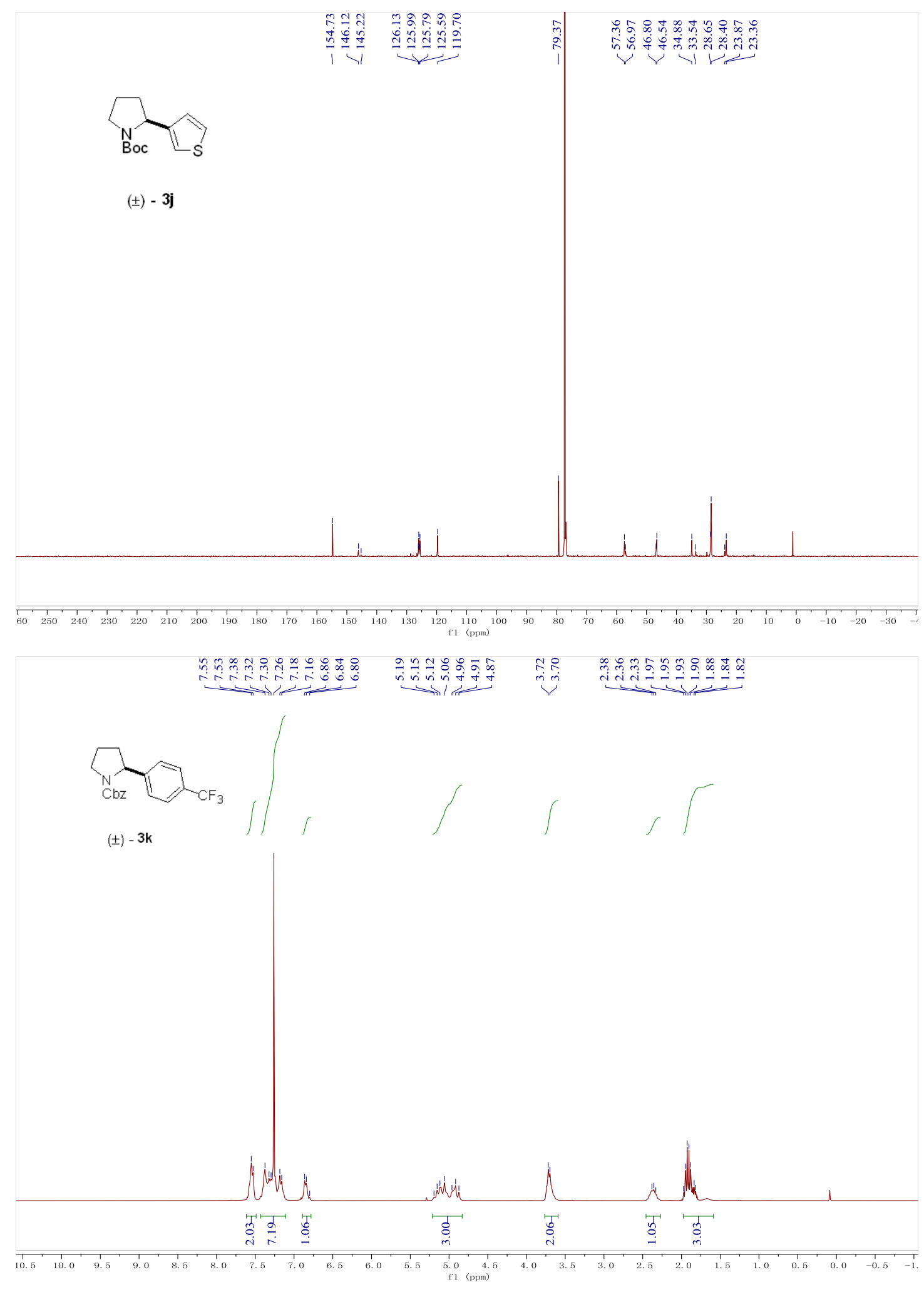



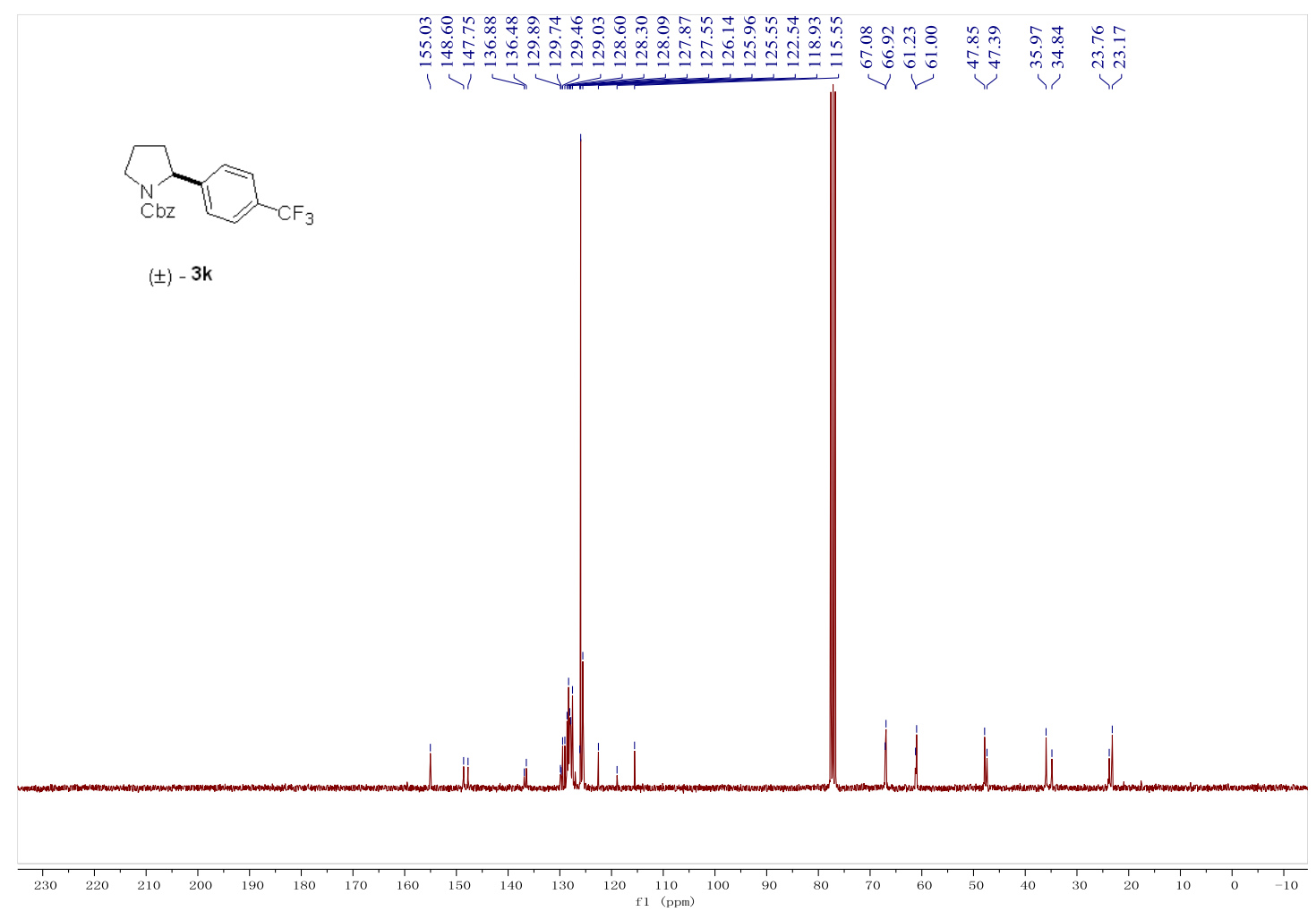

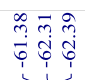

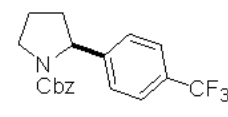

(士) - 3k

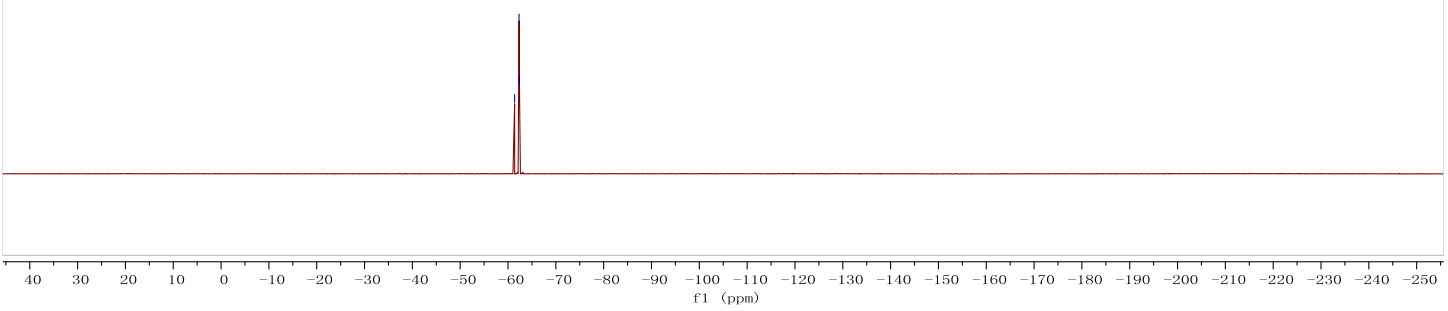




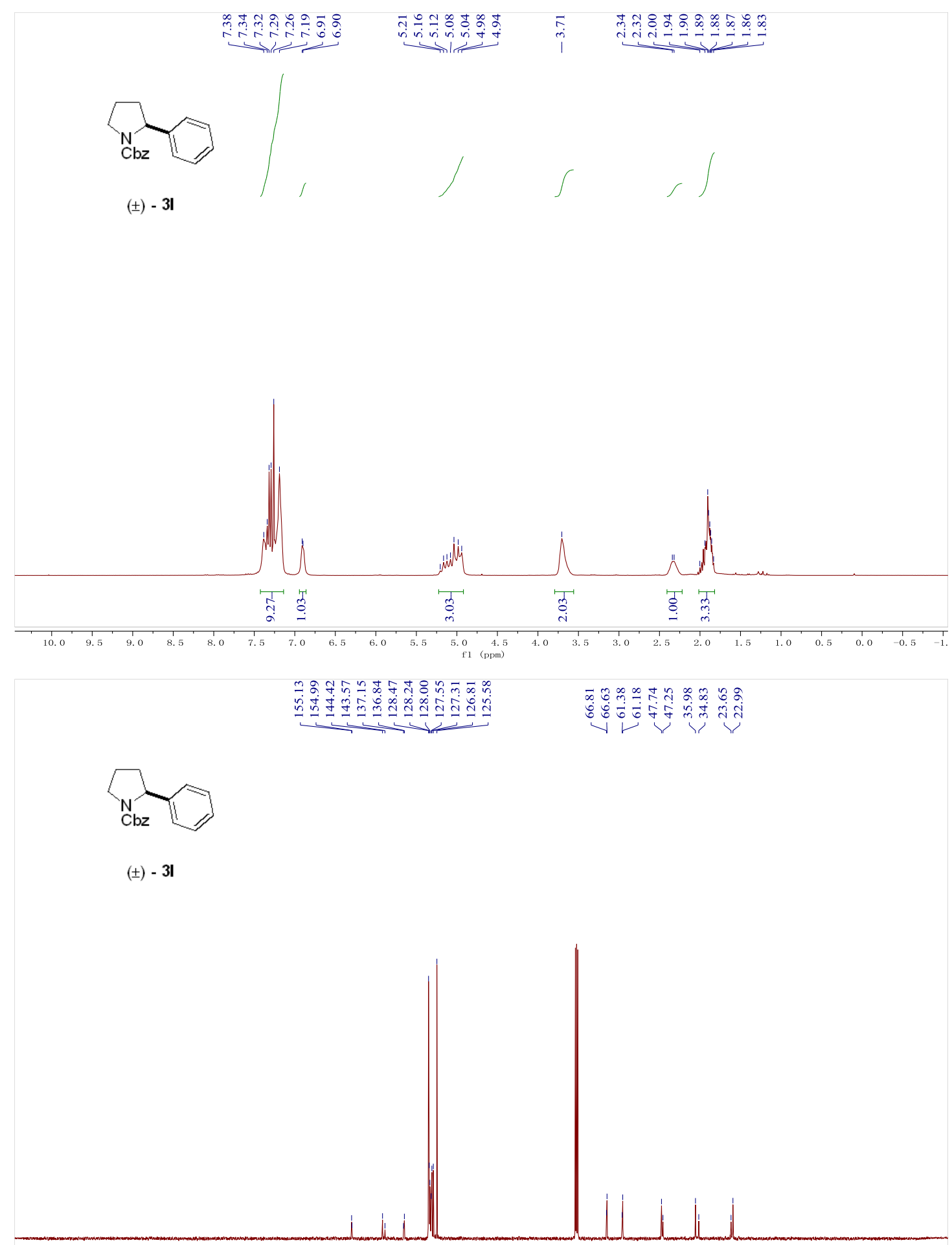

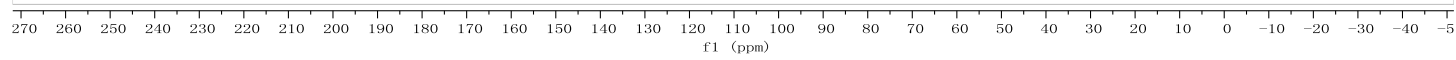




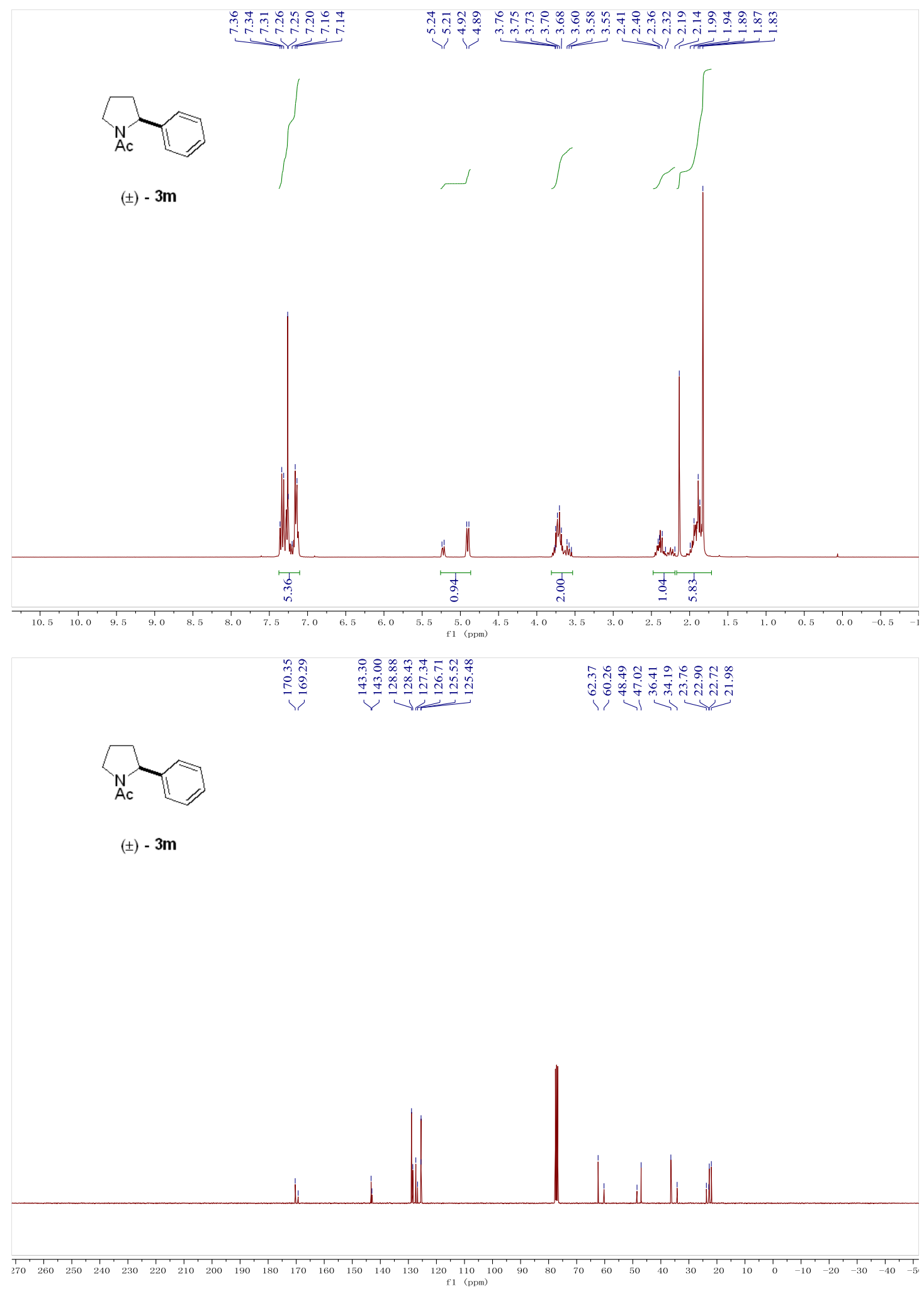




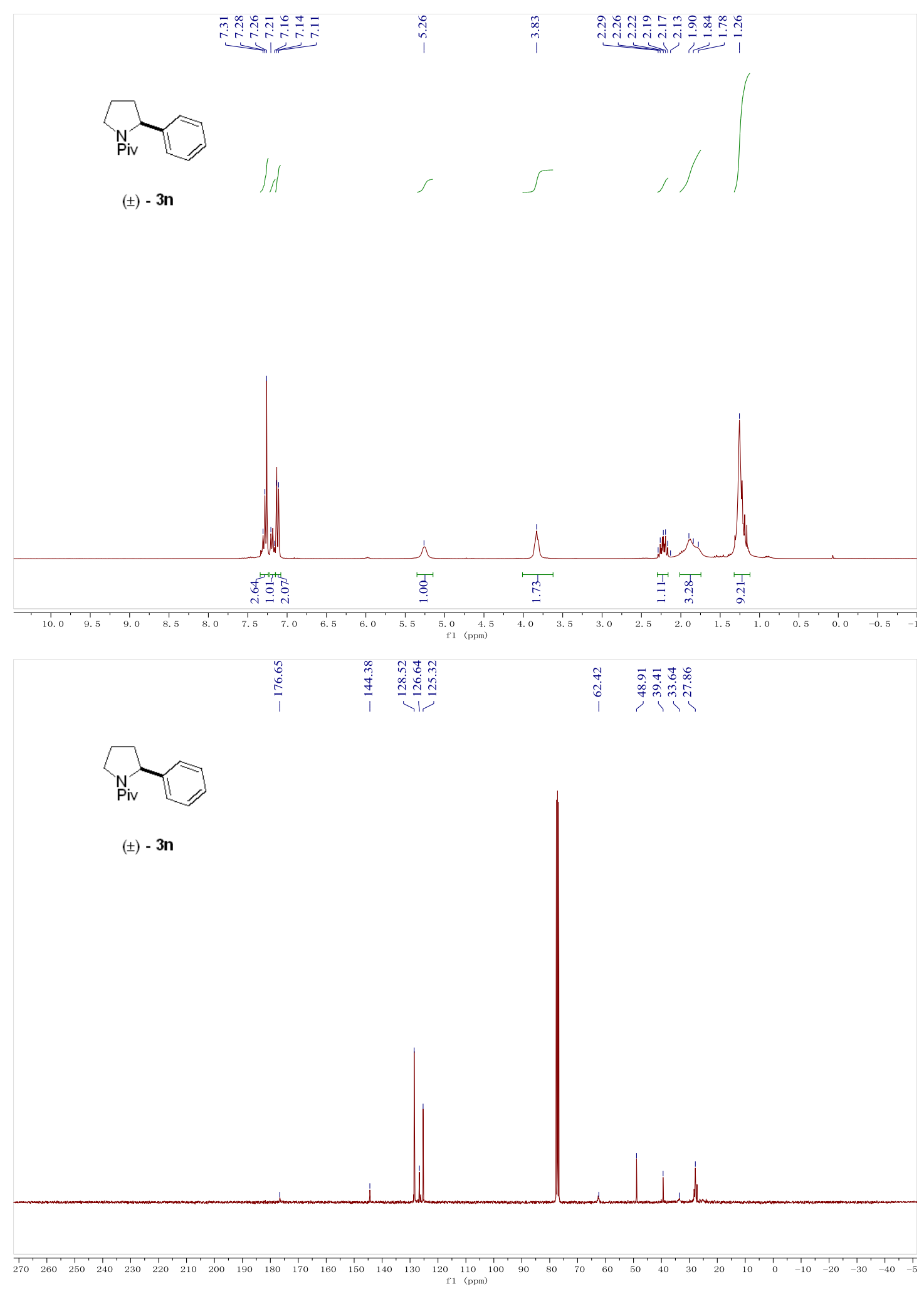




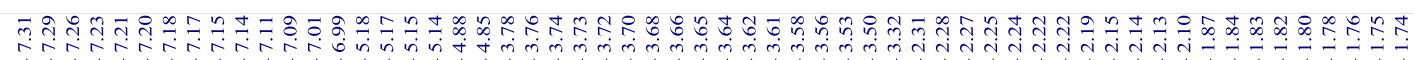

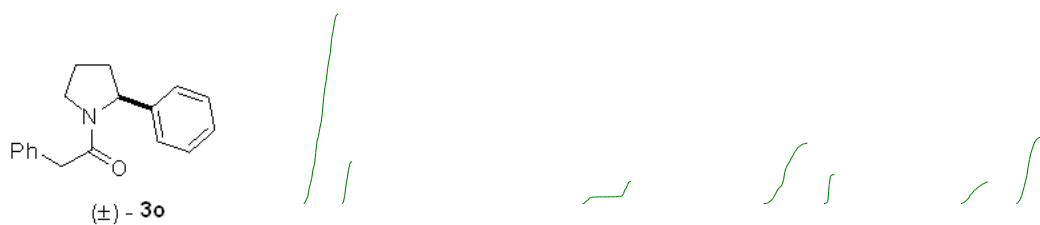

$( \pm)-30$
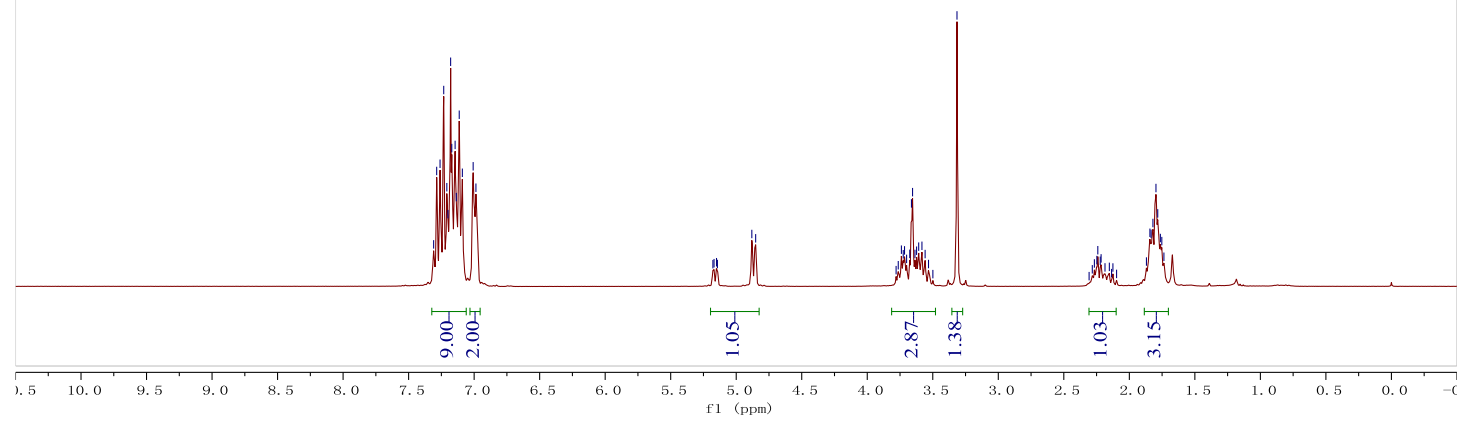

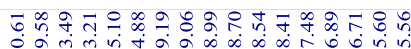

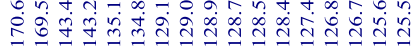

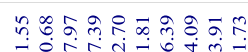

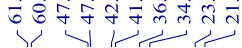

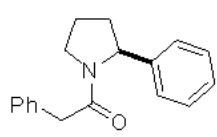

$( \pm)-30$
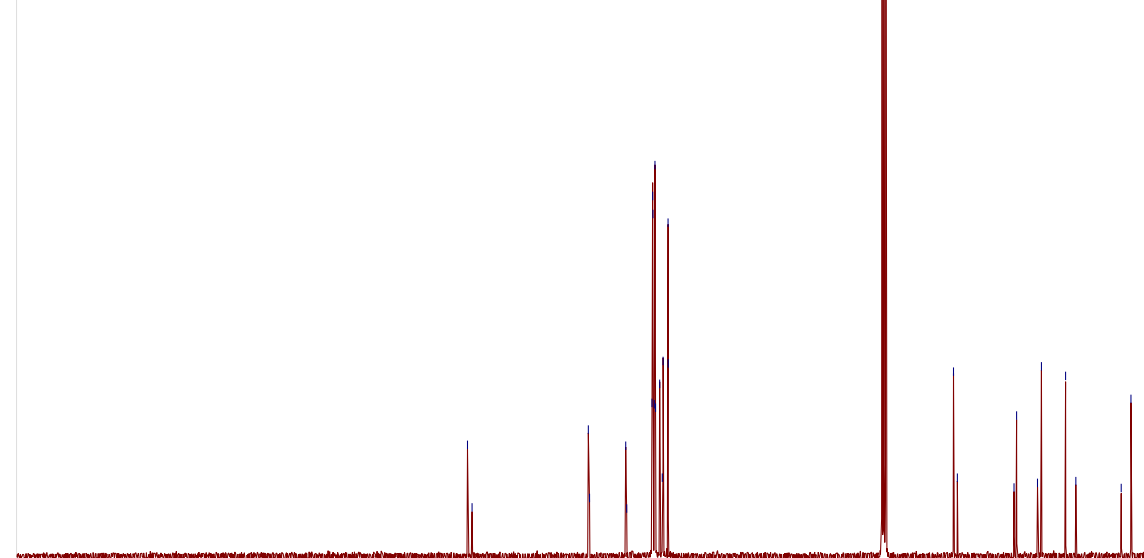

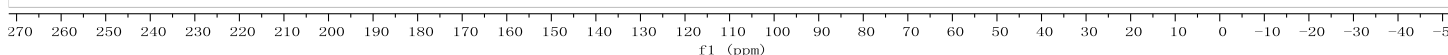




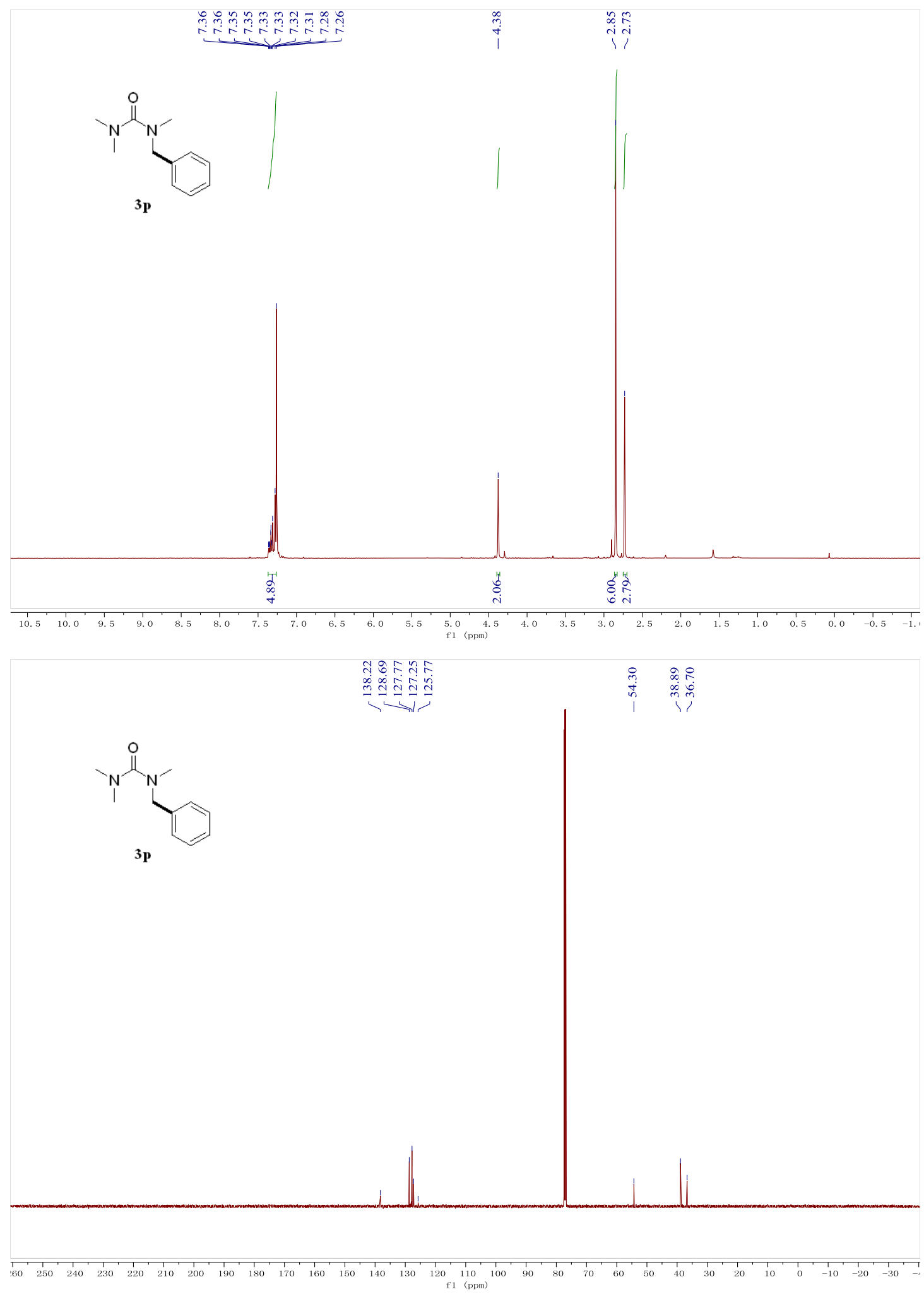




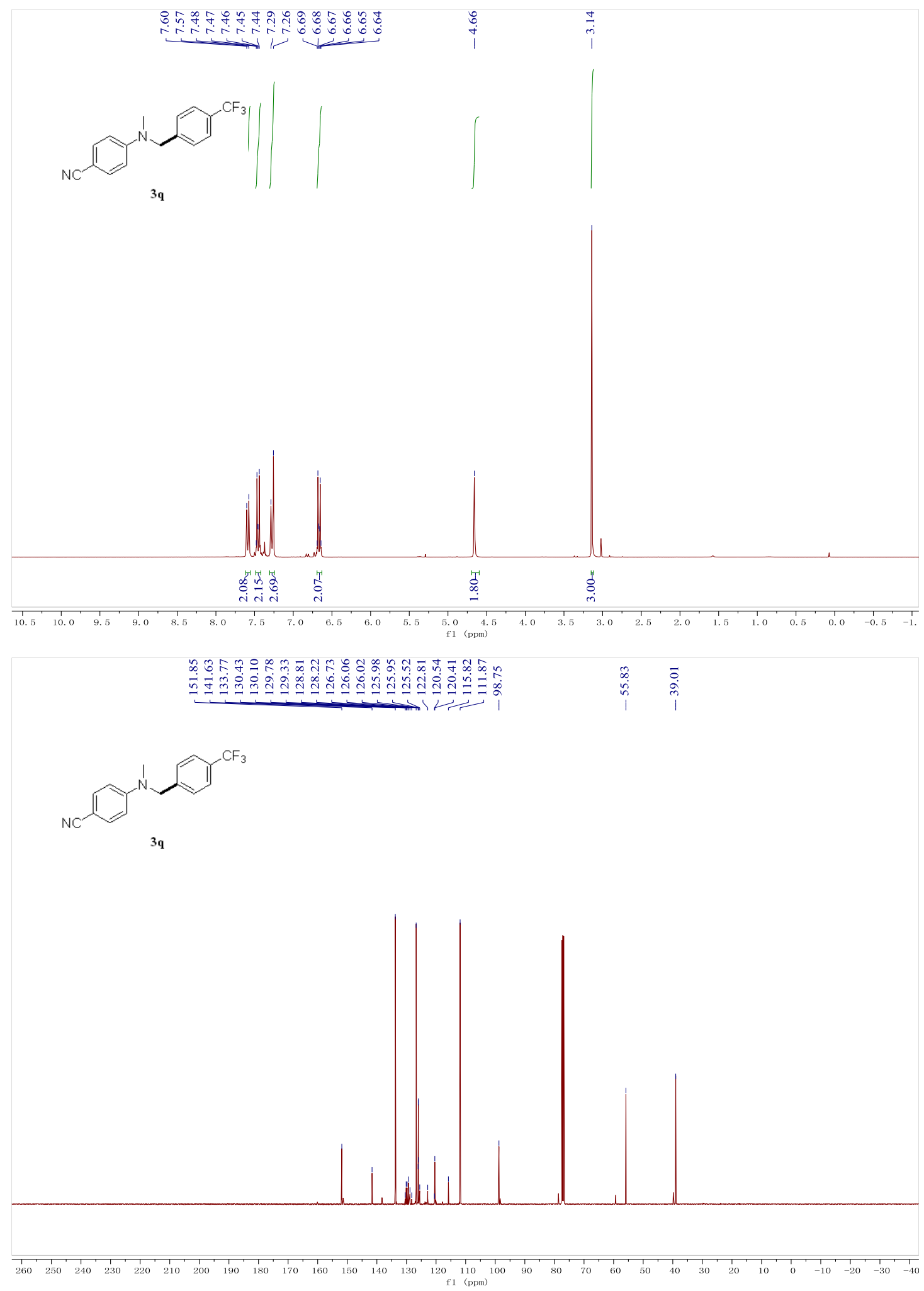




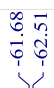

(N)

$3 q$

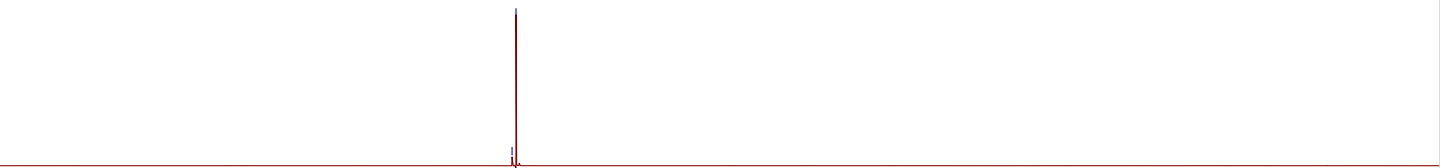

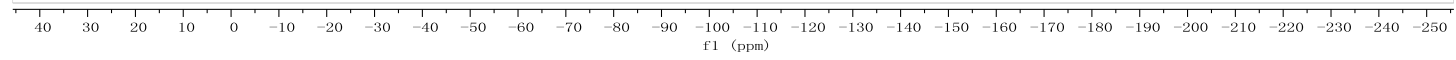

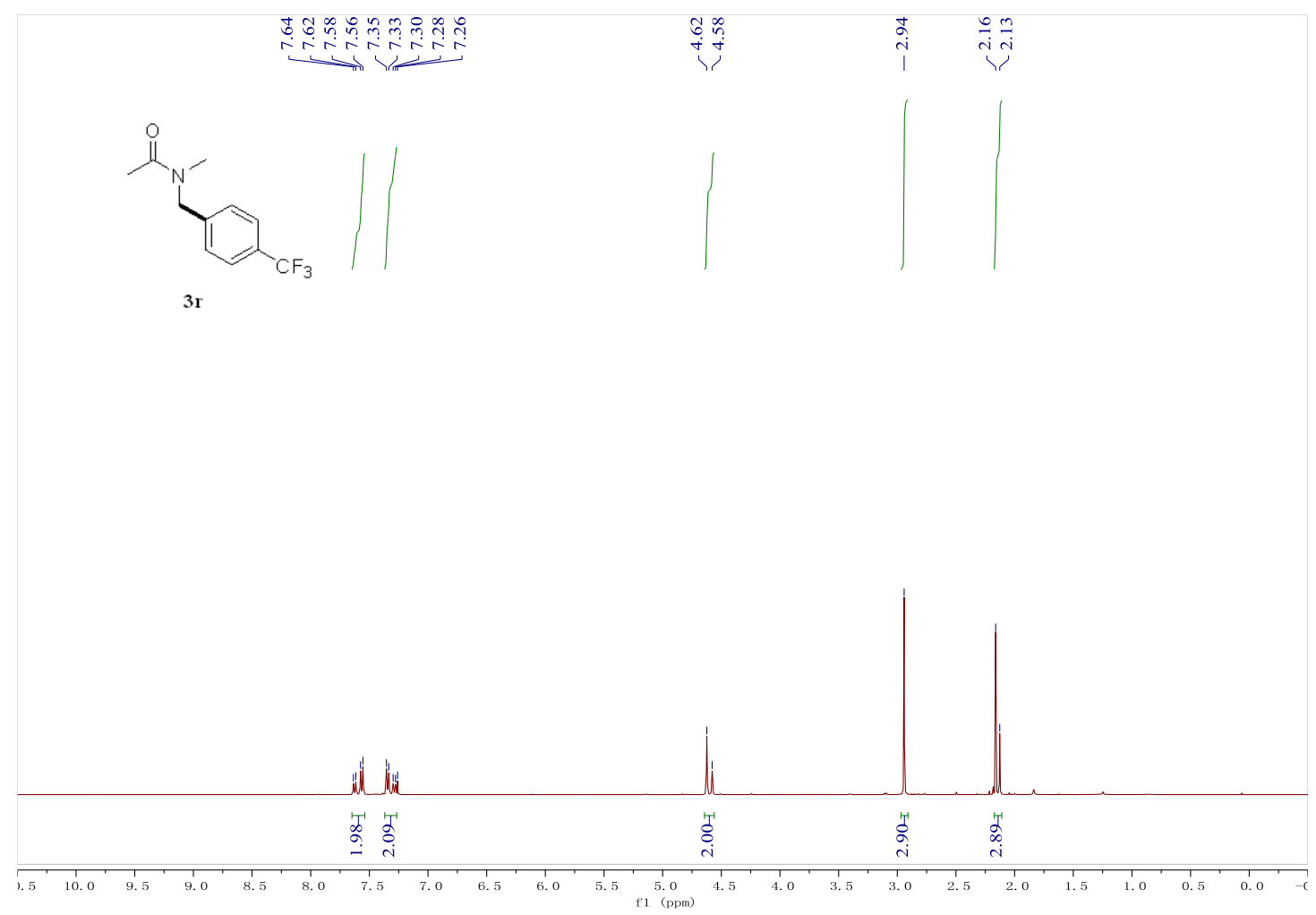




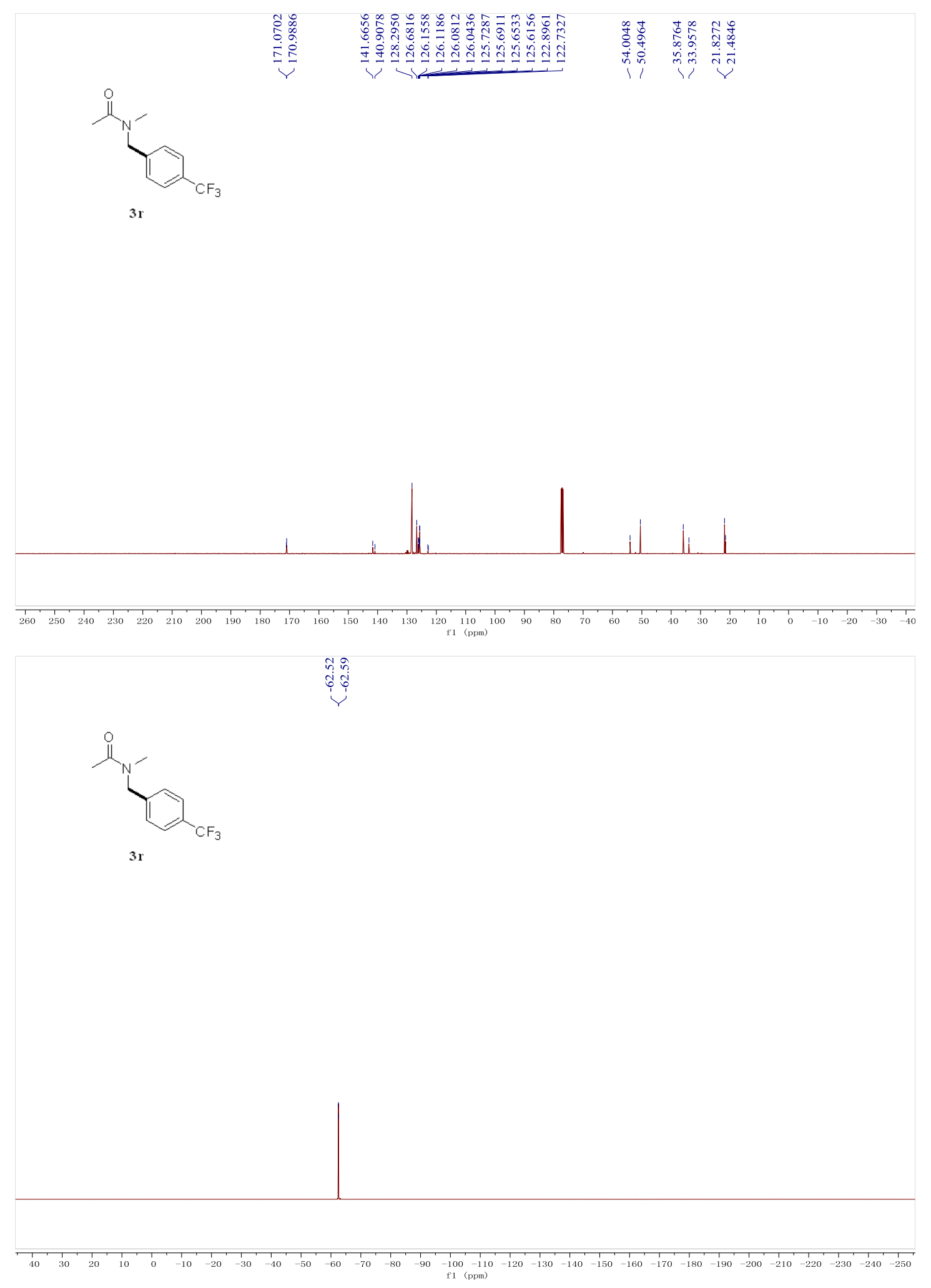




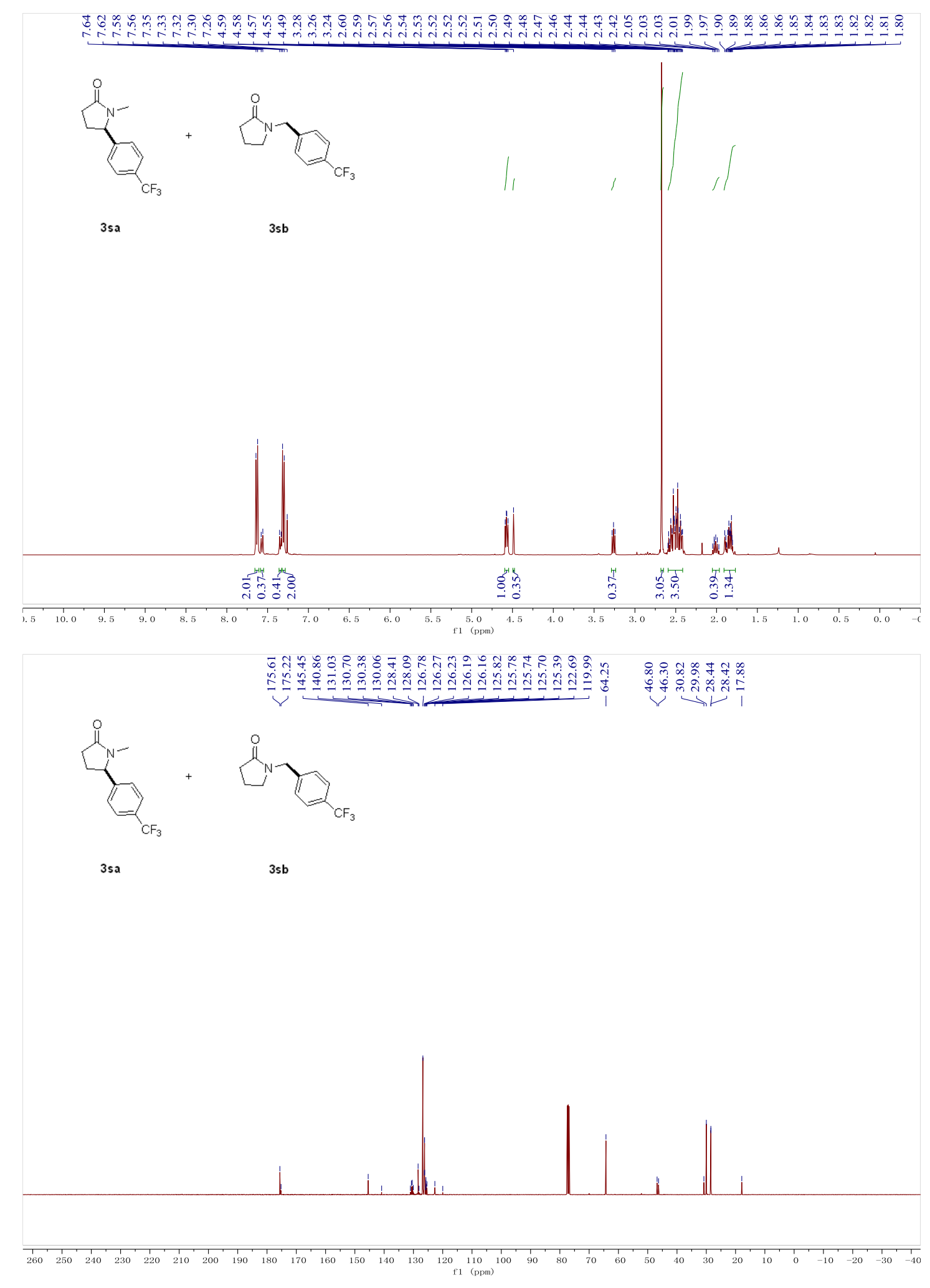




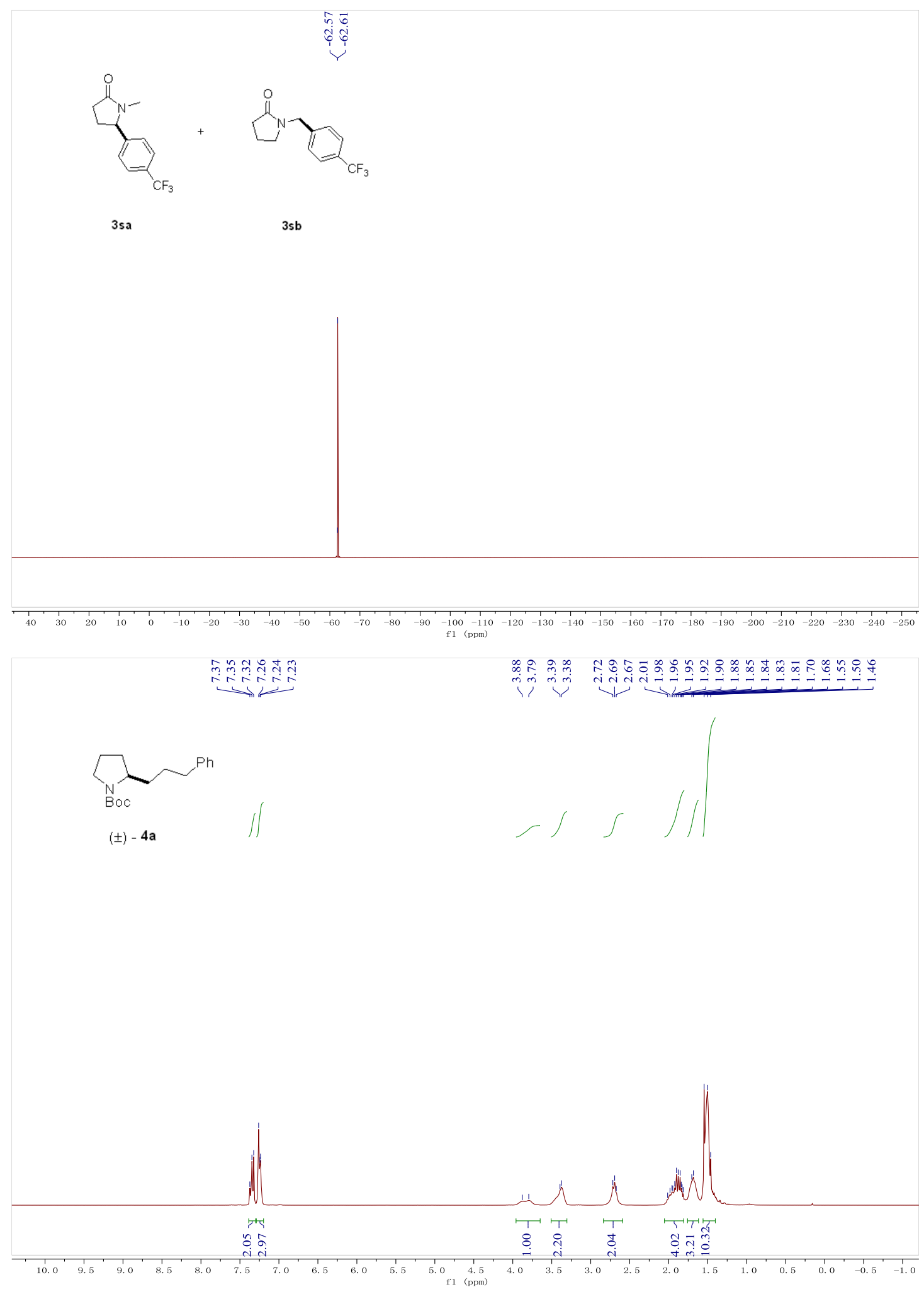




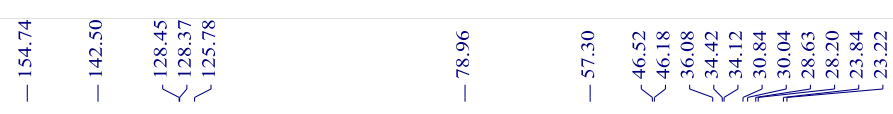

$\bigcup_{\substack{N \\ B O C}}^{P h}$

(士) $-4 a$
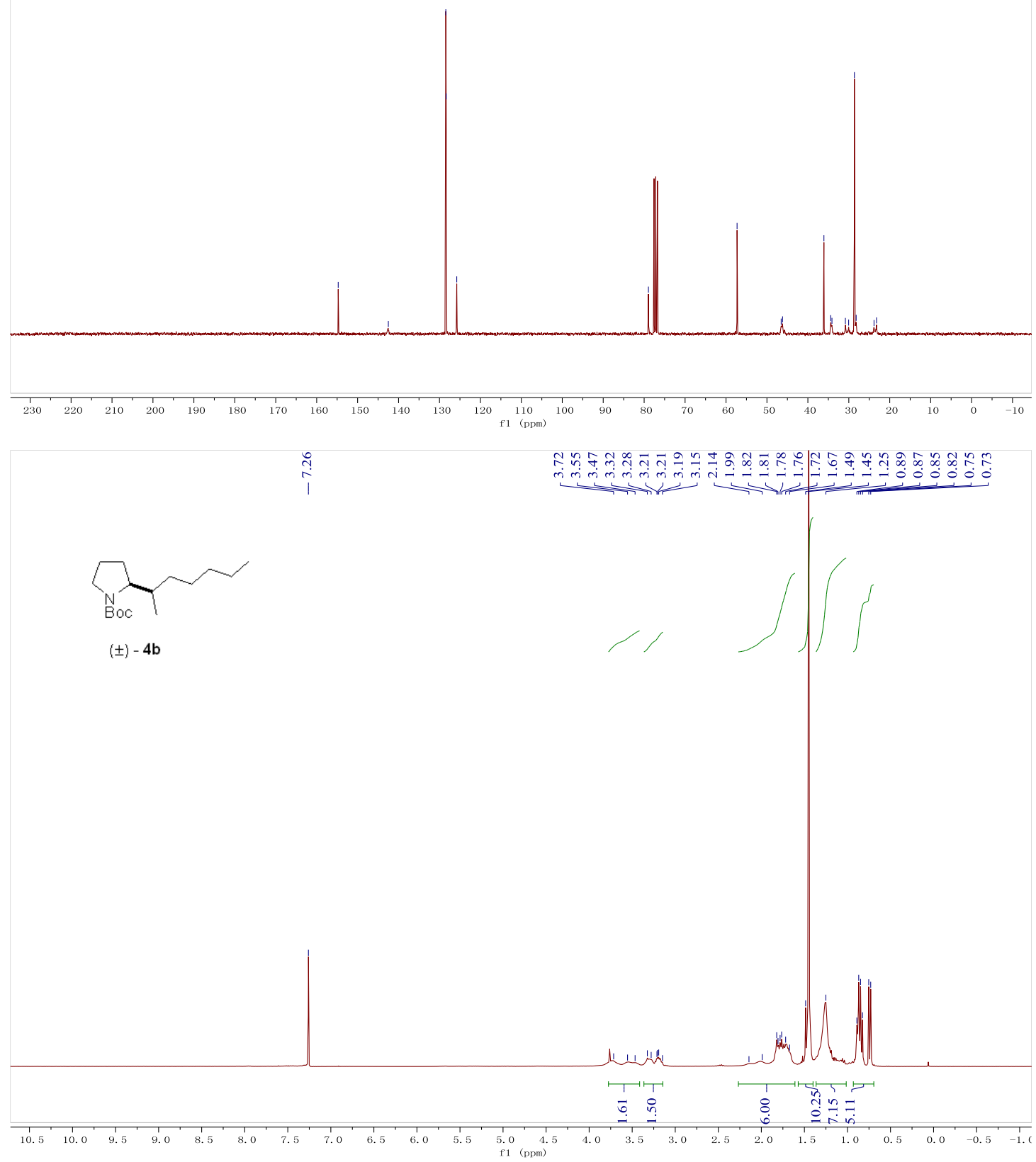


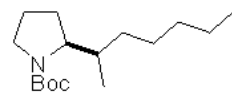

$( \pm)-4 b$

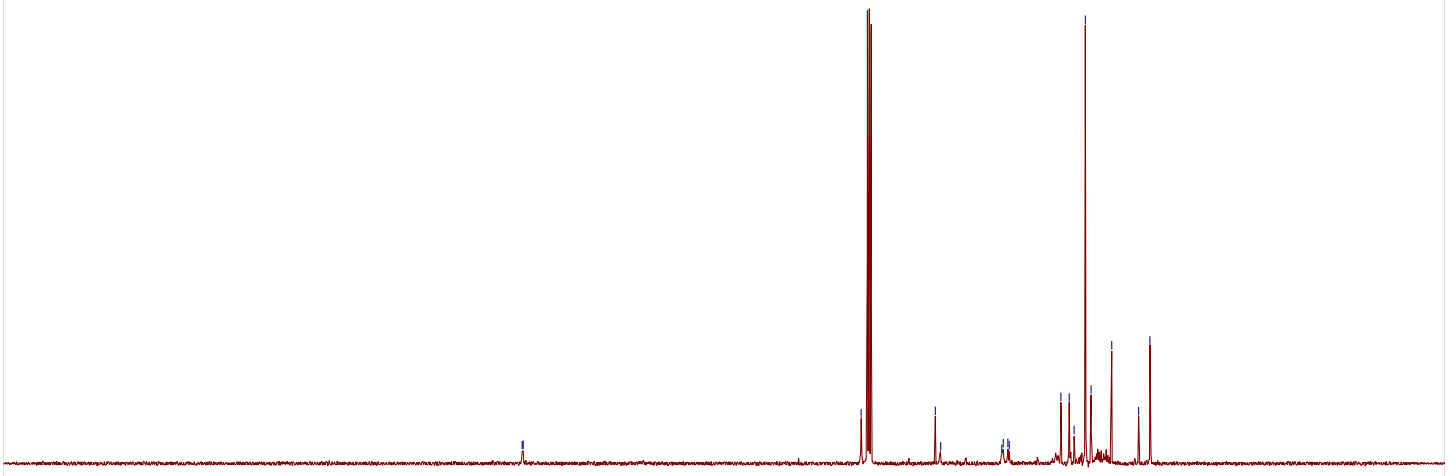

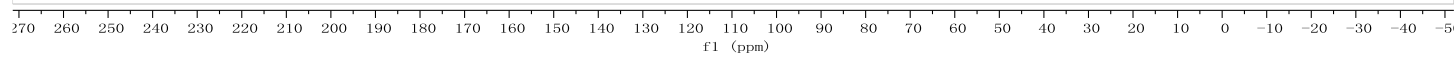

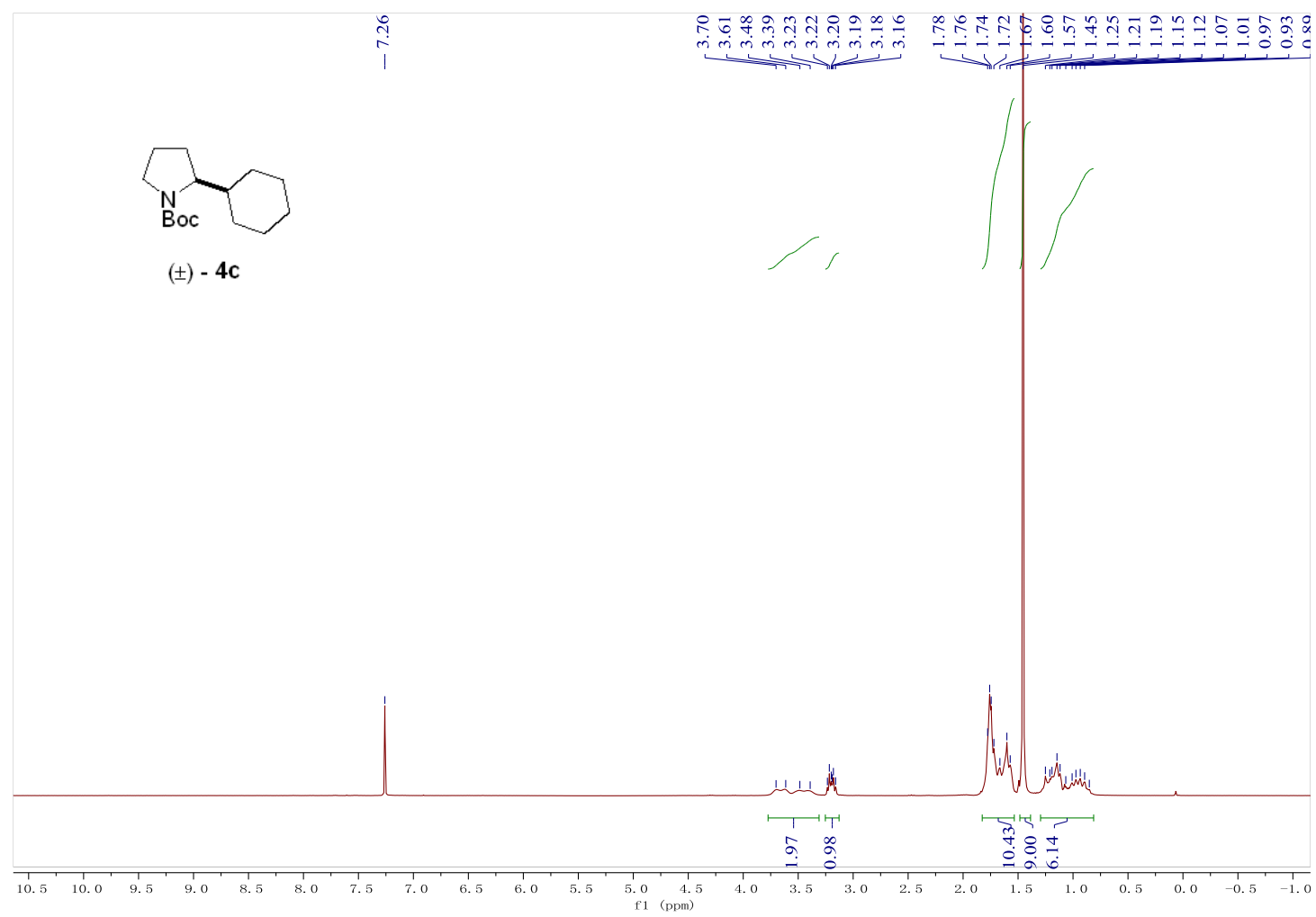




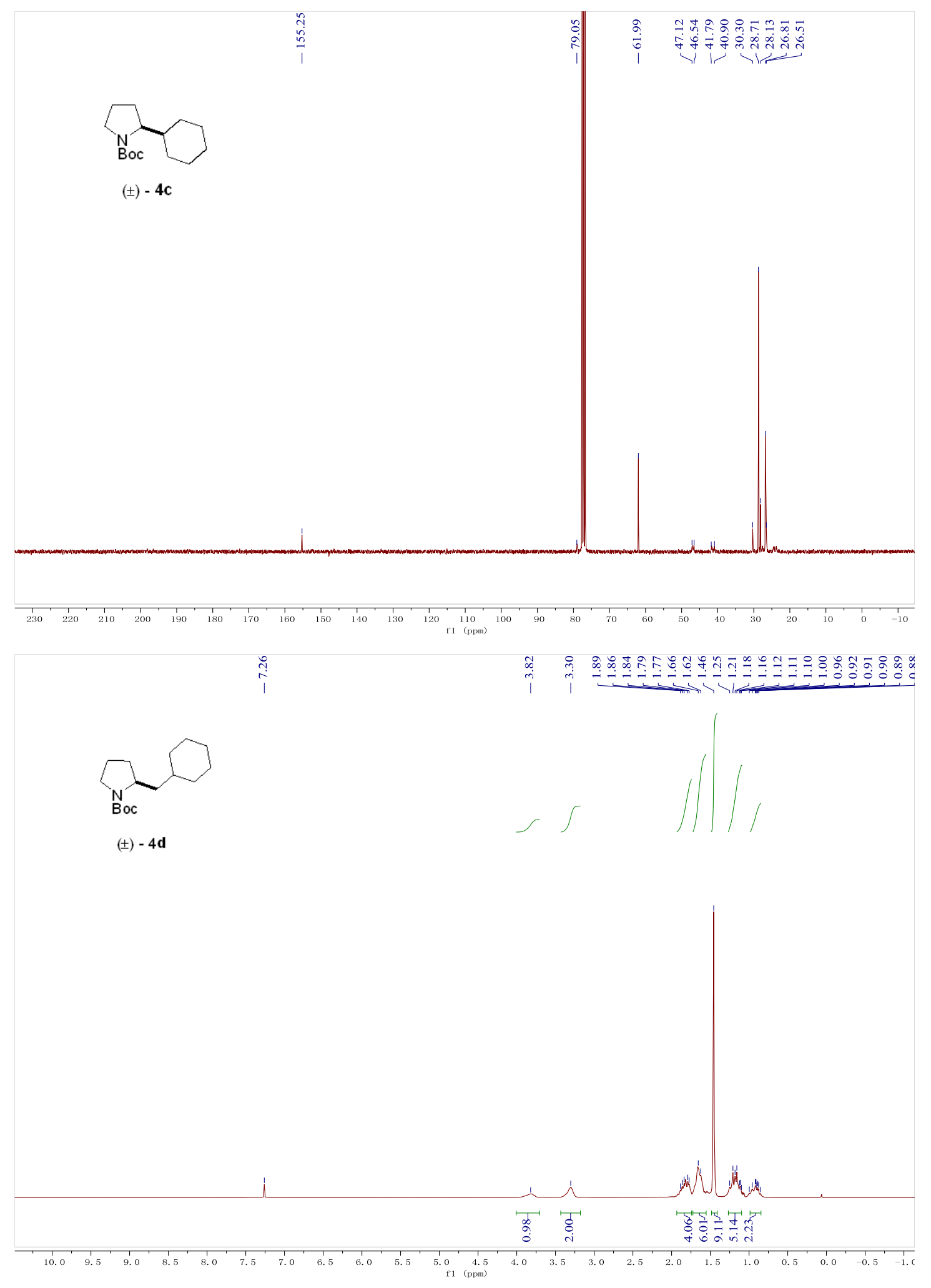




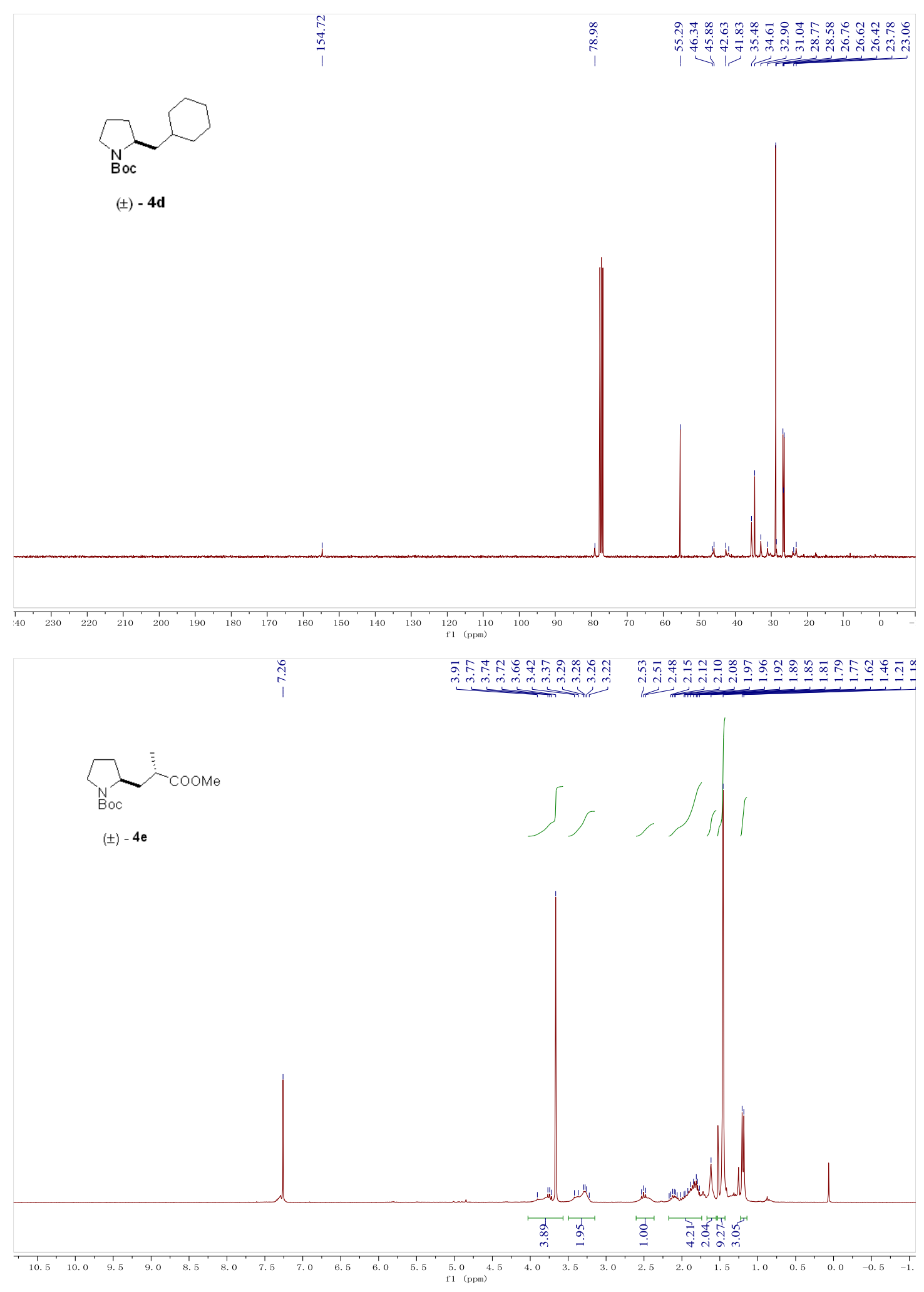




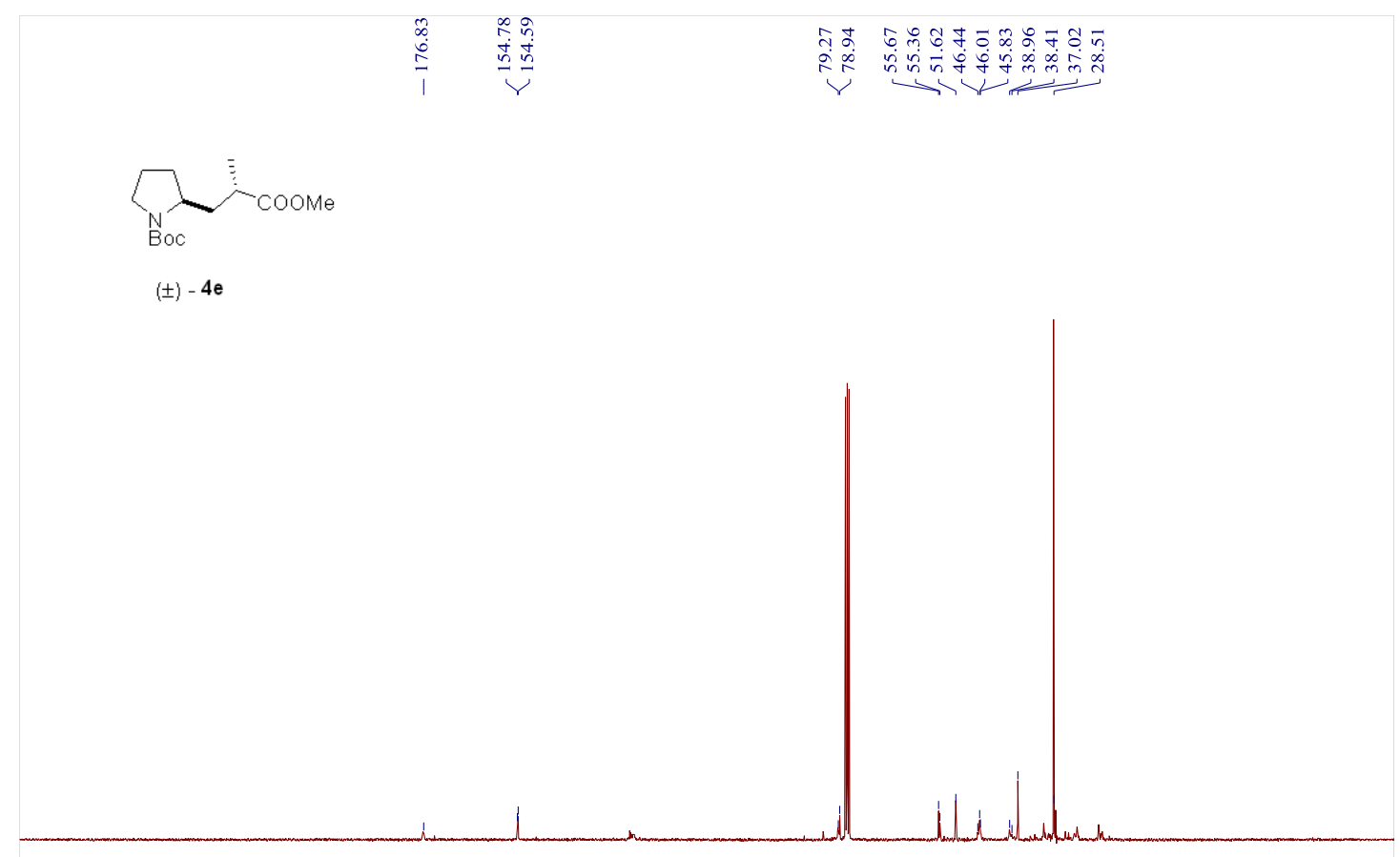

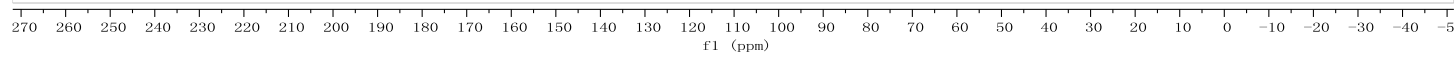

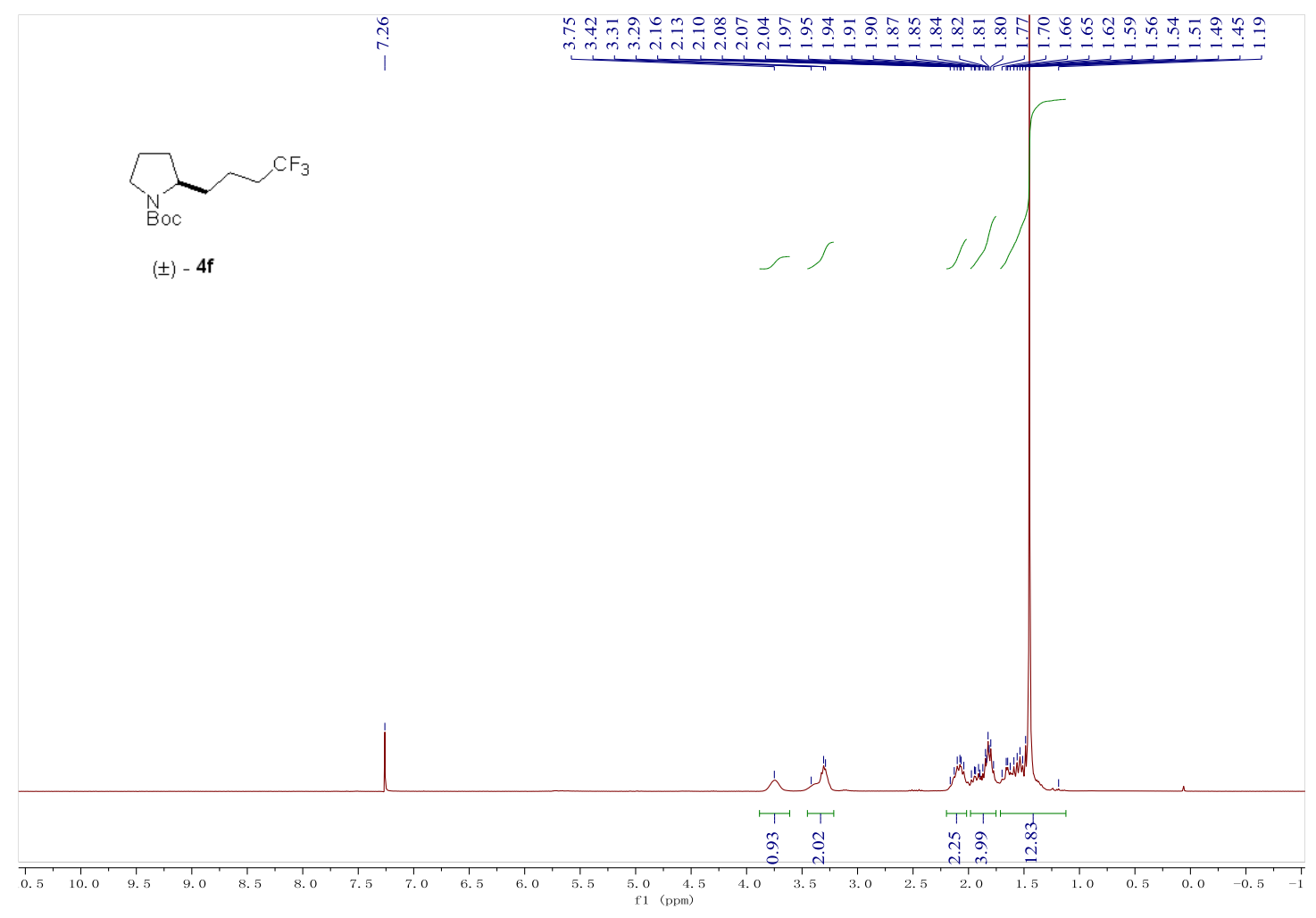




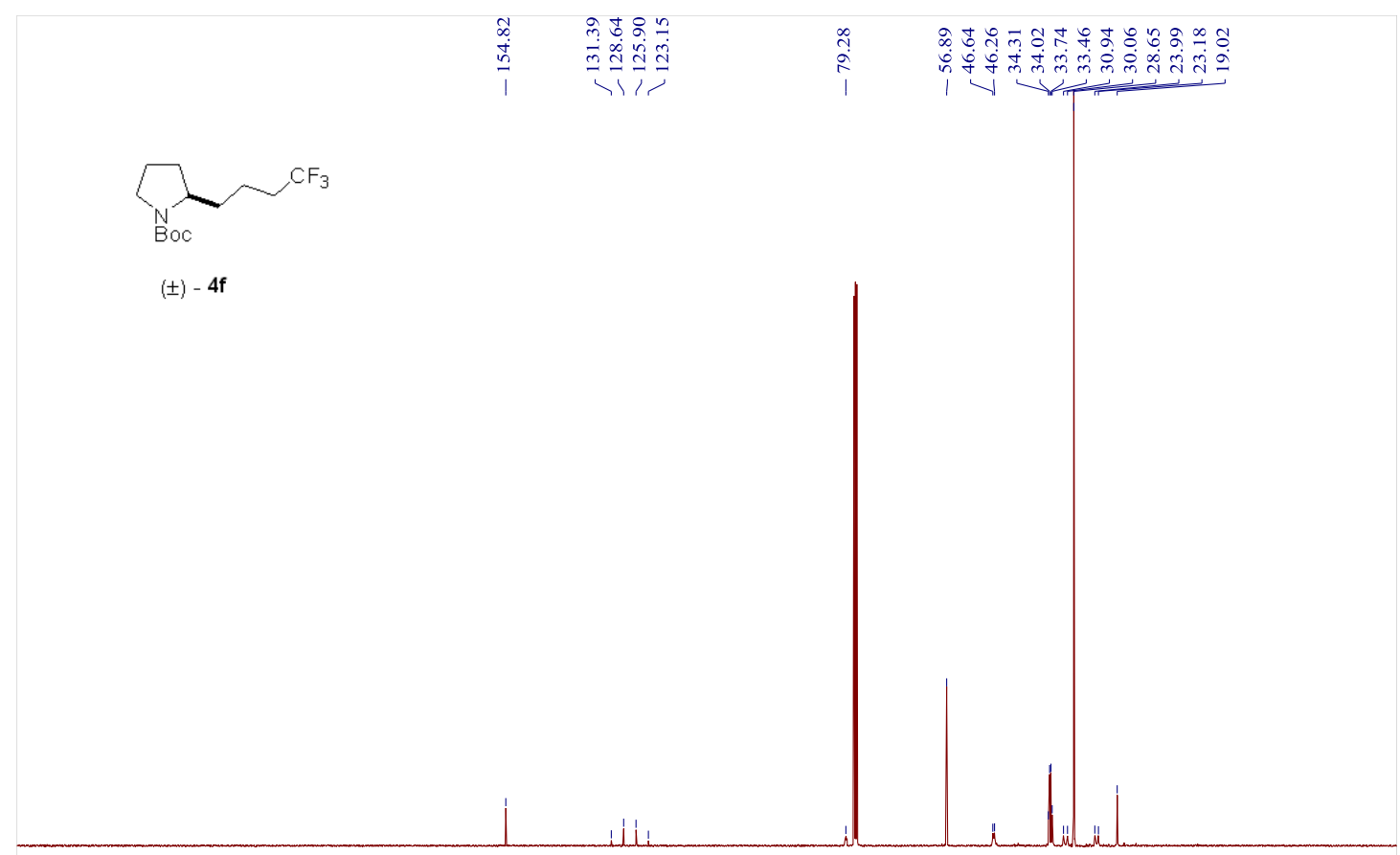

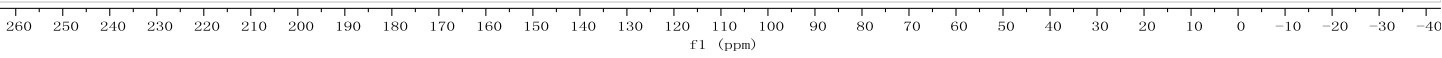

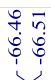

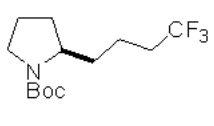

( \pm ) - $4 f$

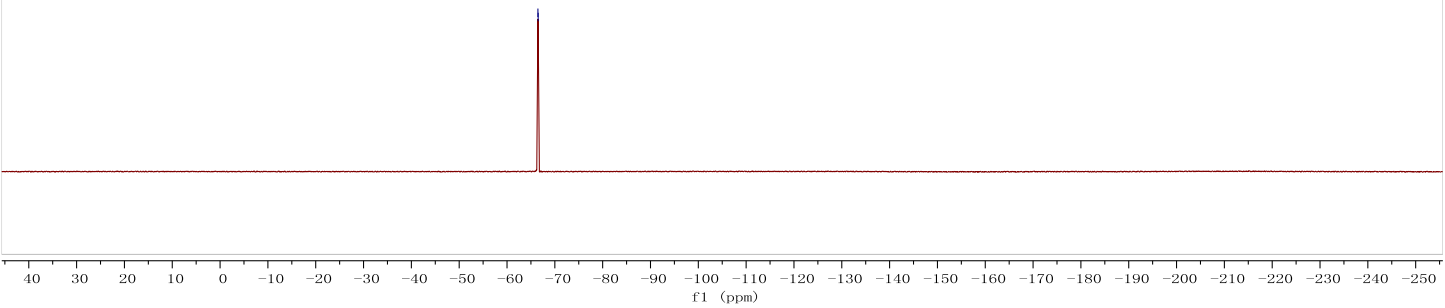




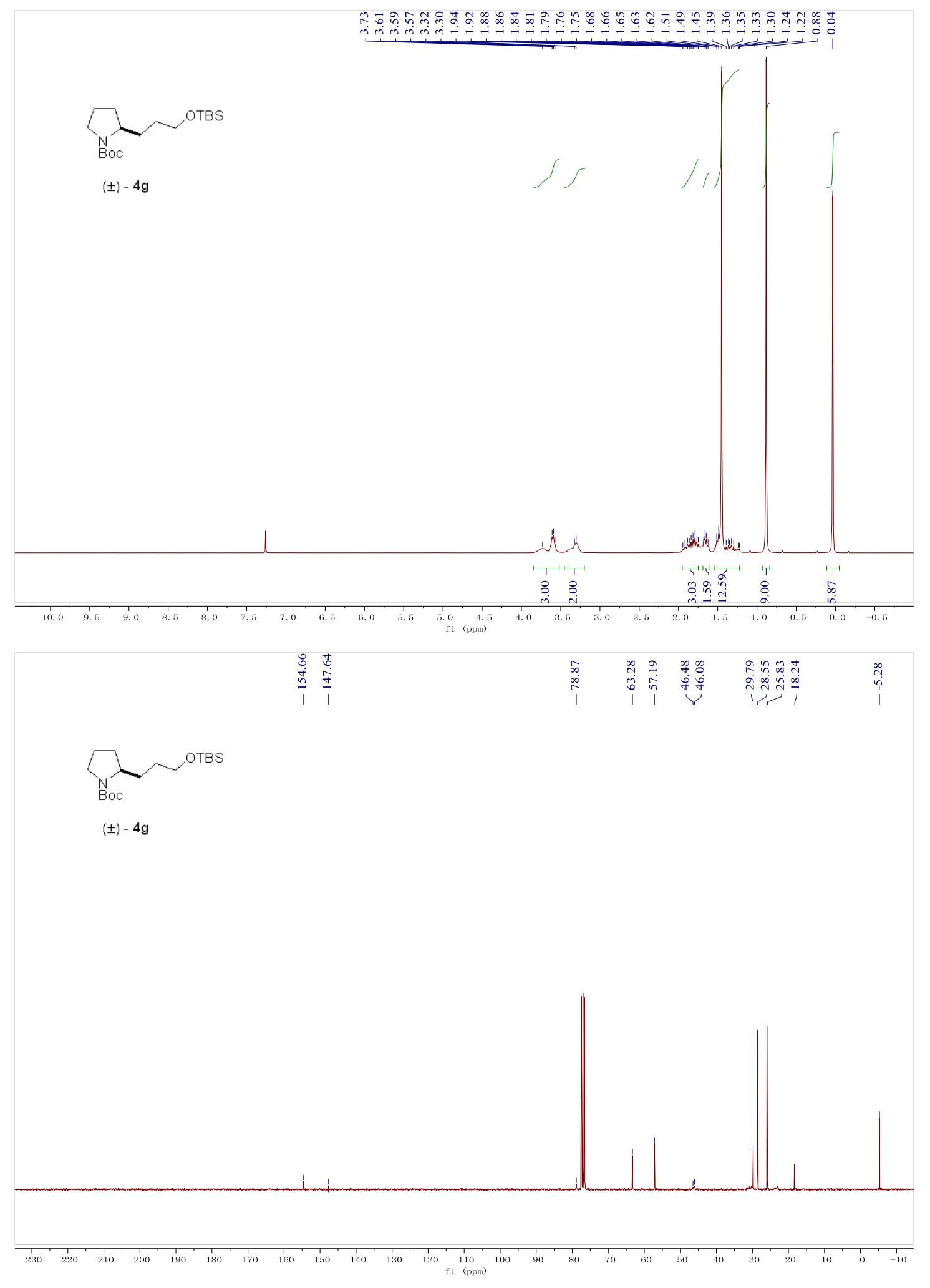




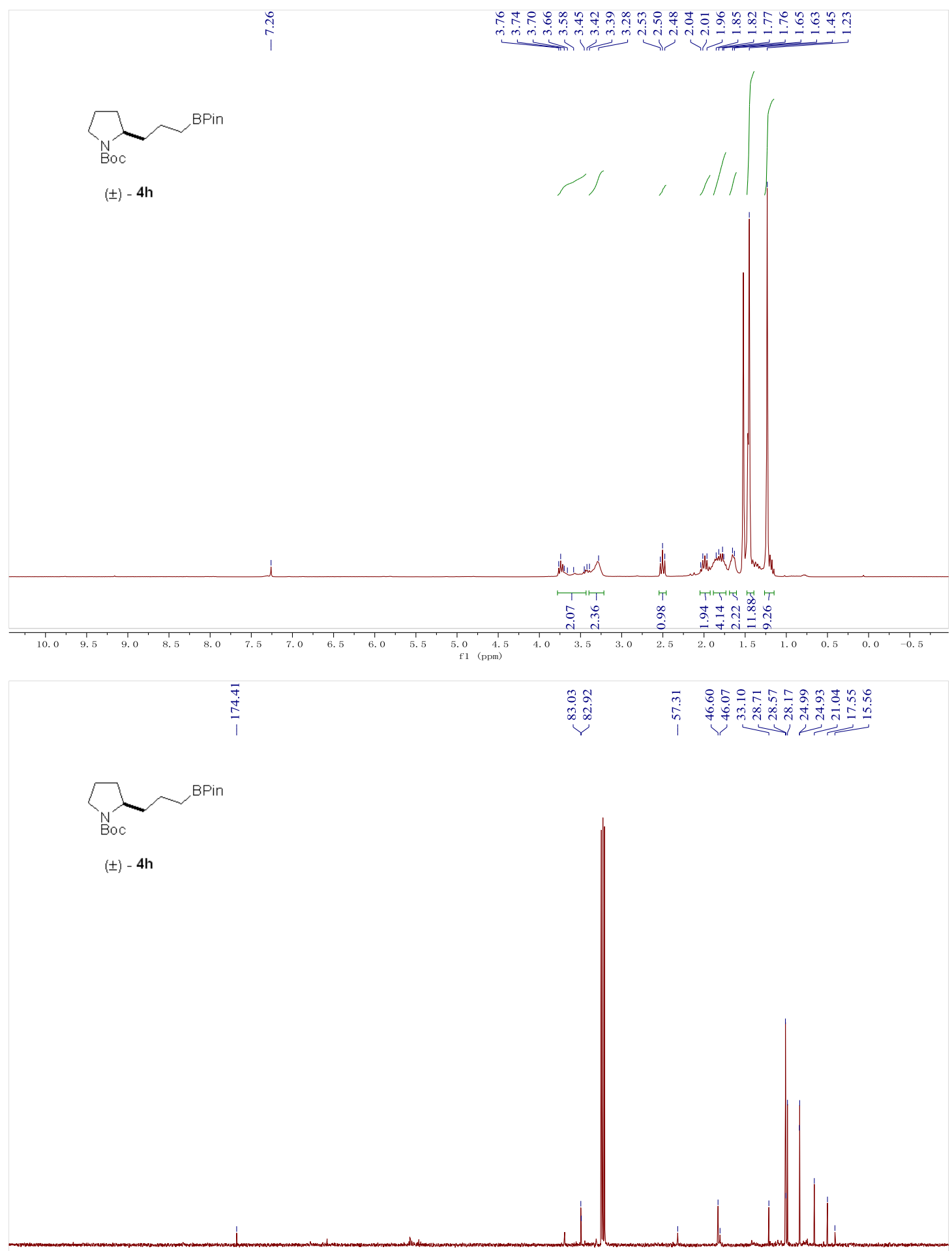

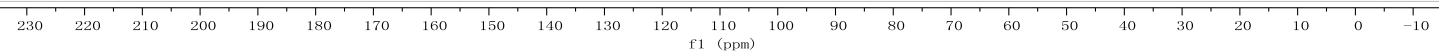




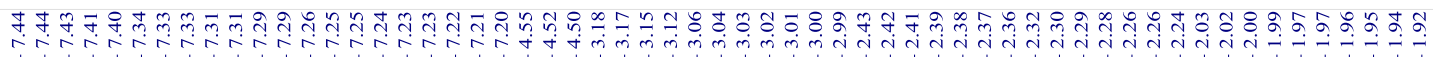

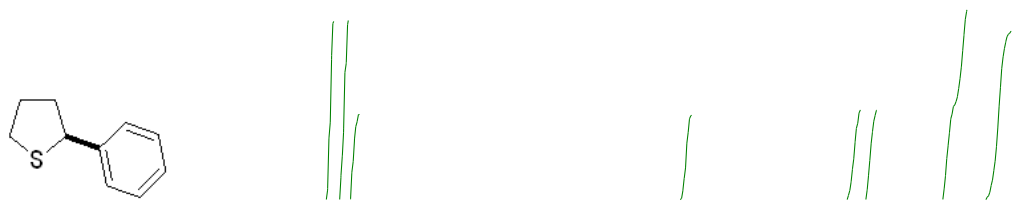

$6 a$

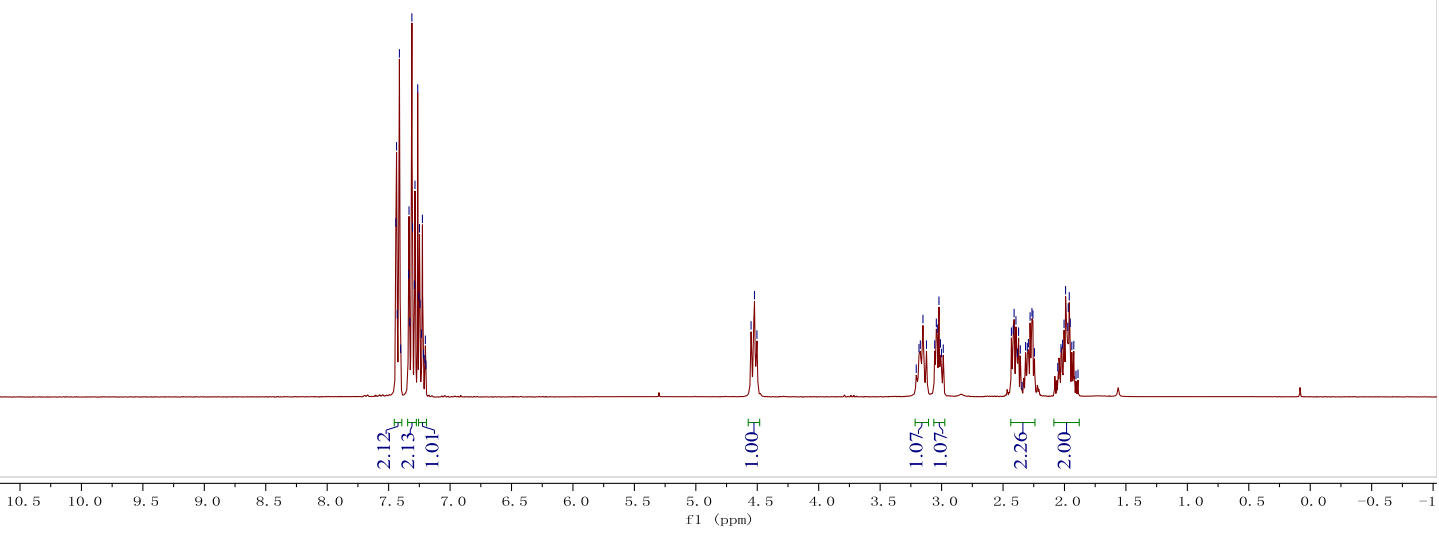

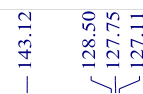

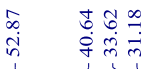

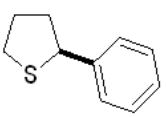

$6 a$

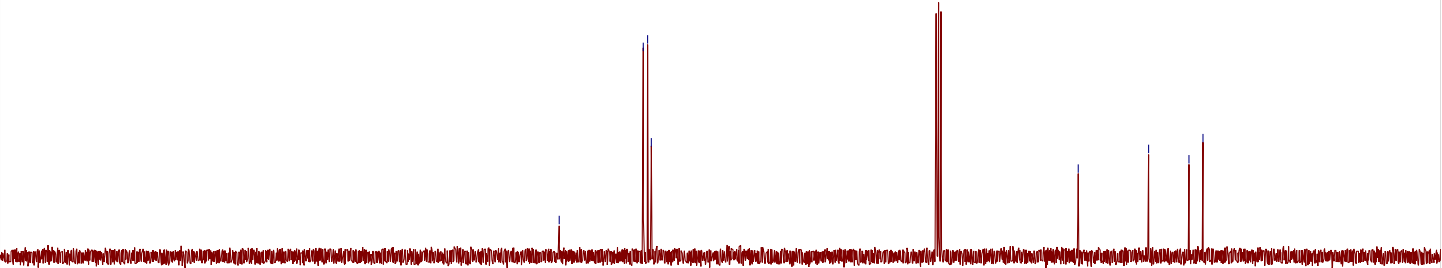

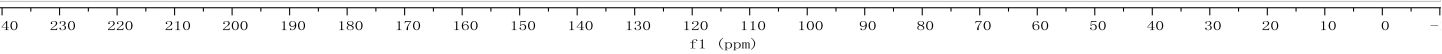




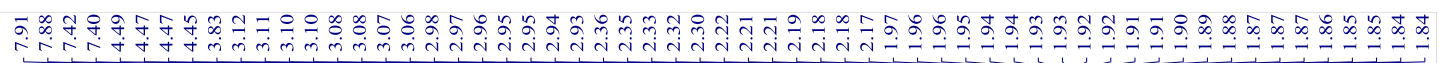

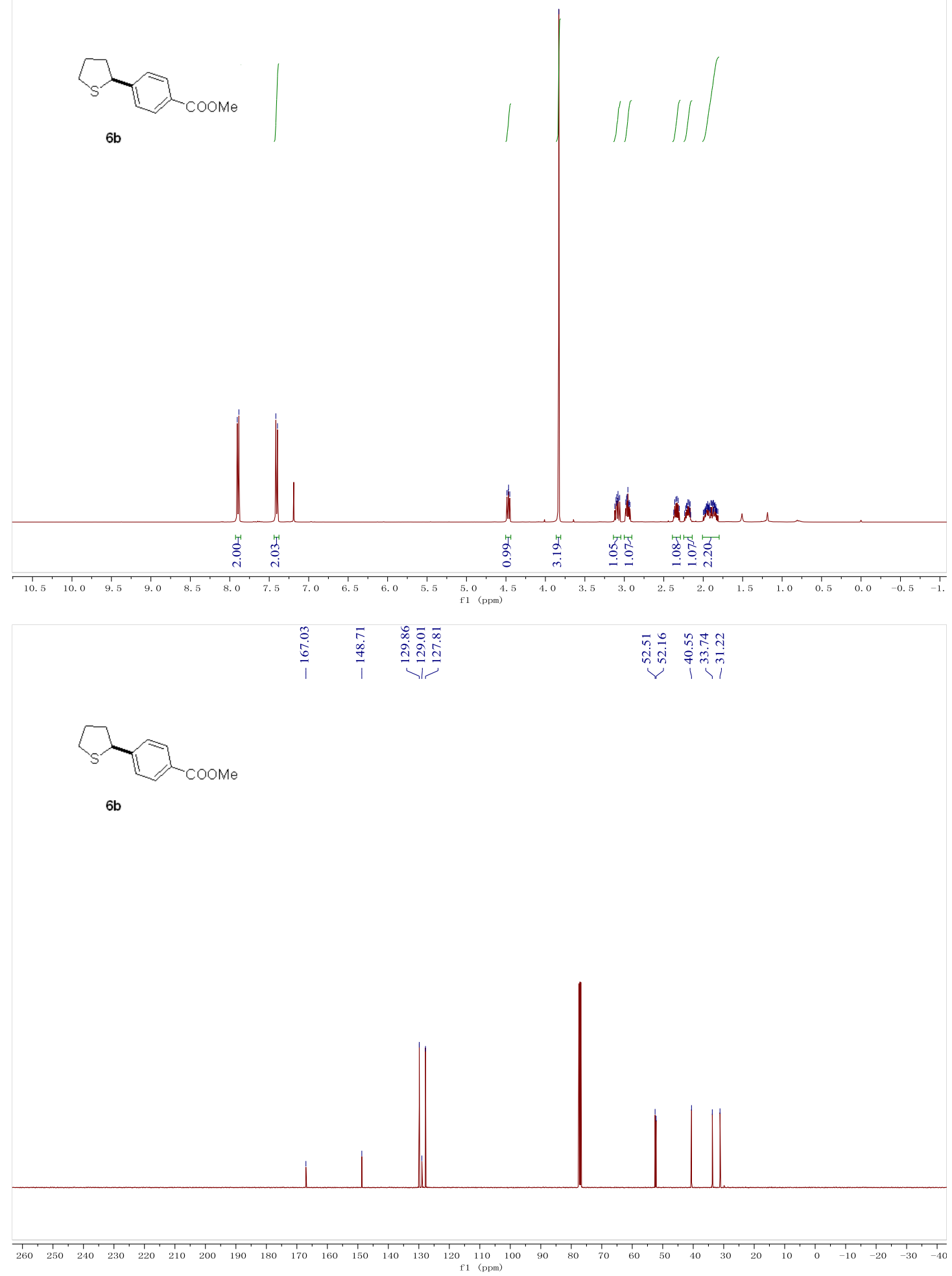




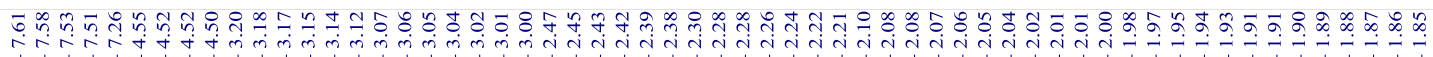

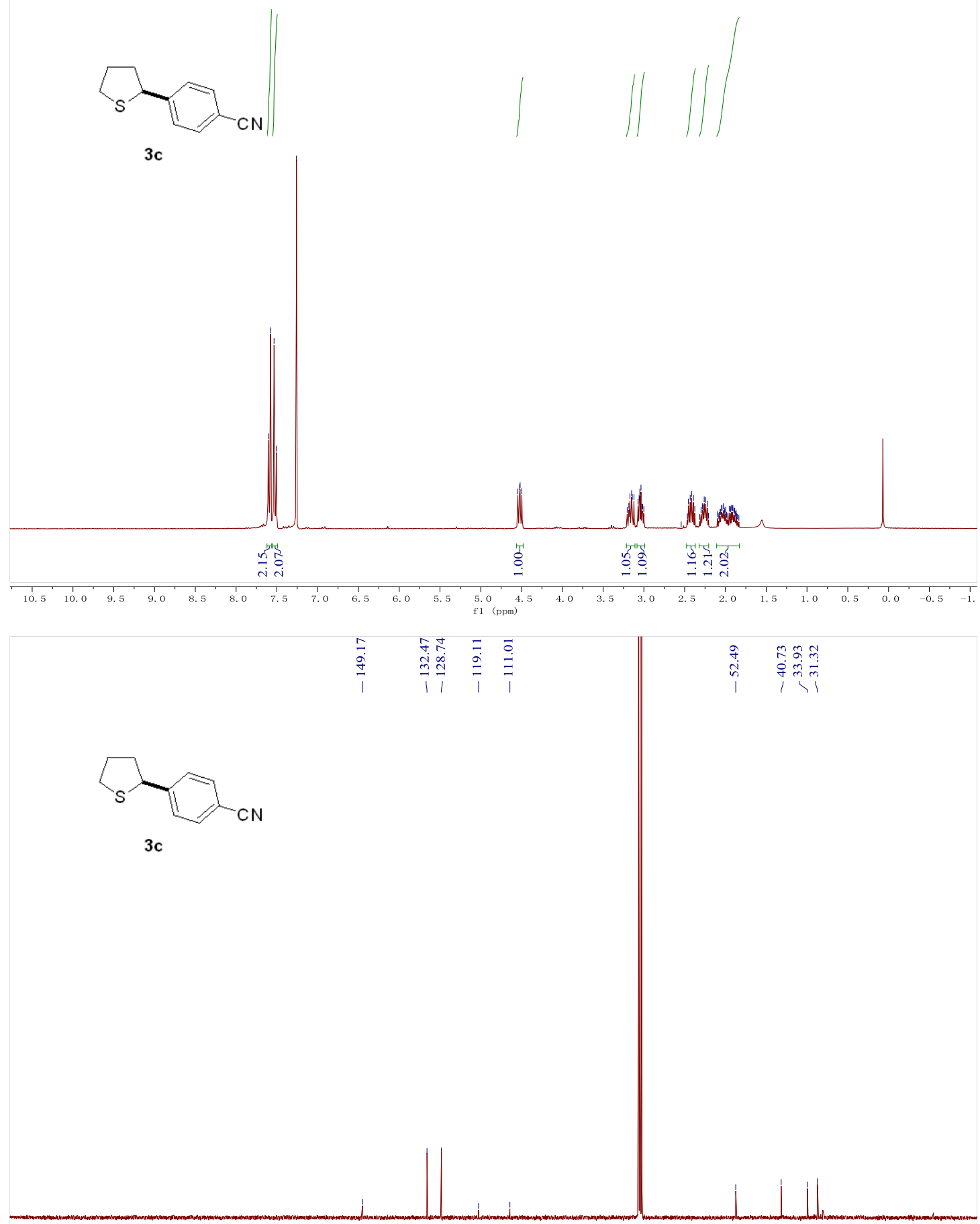

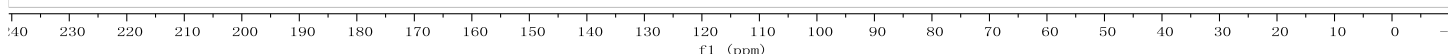




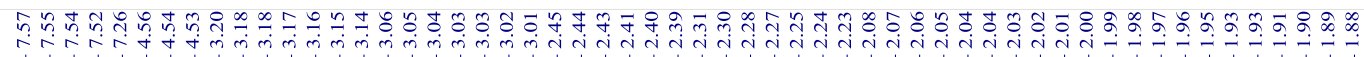

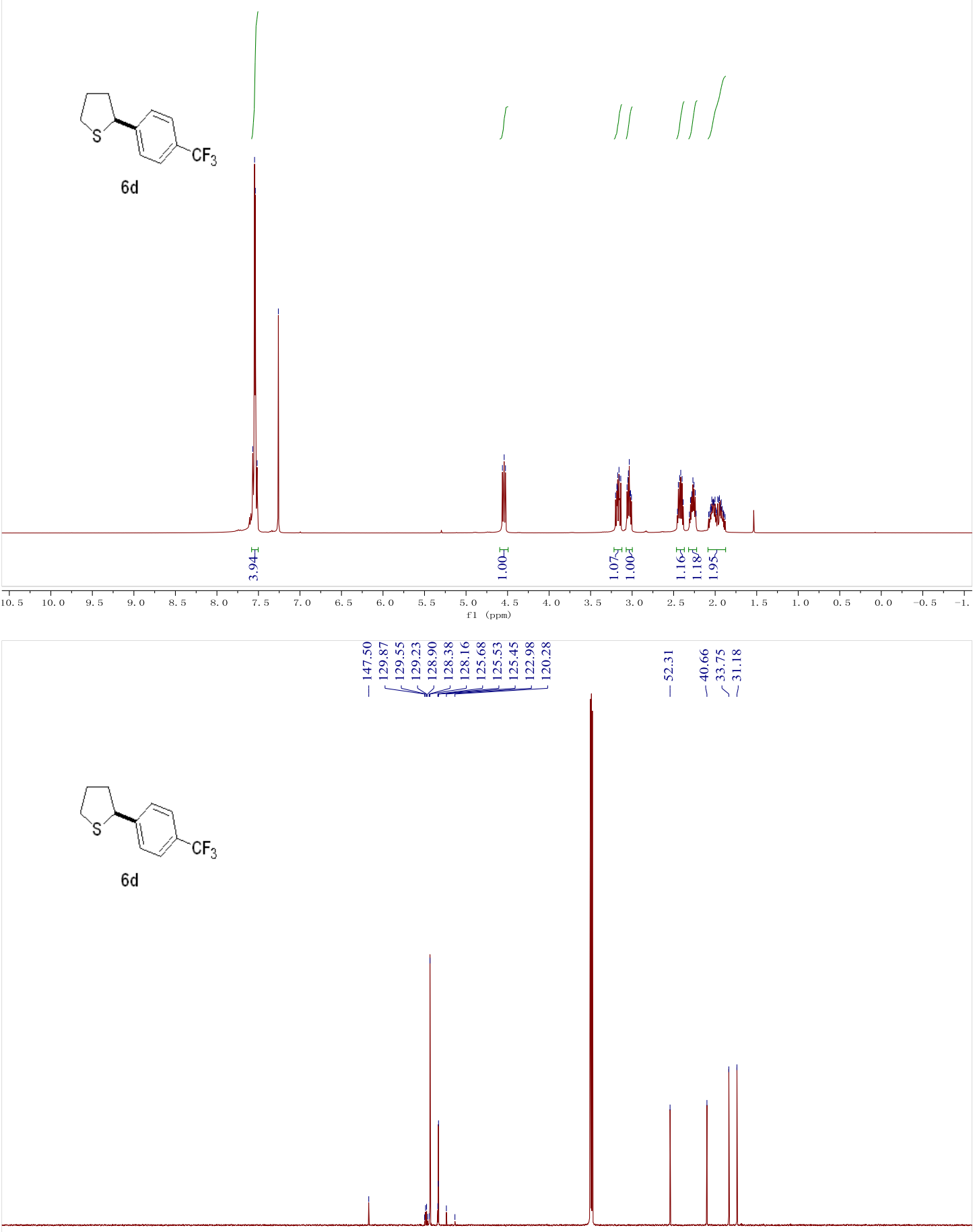

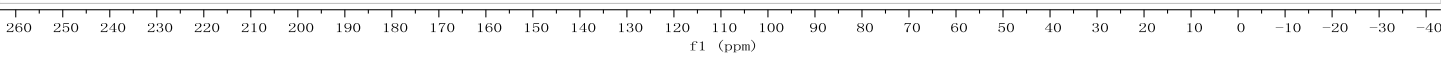




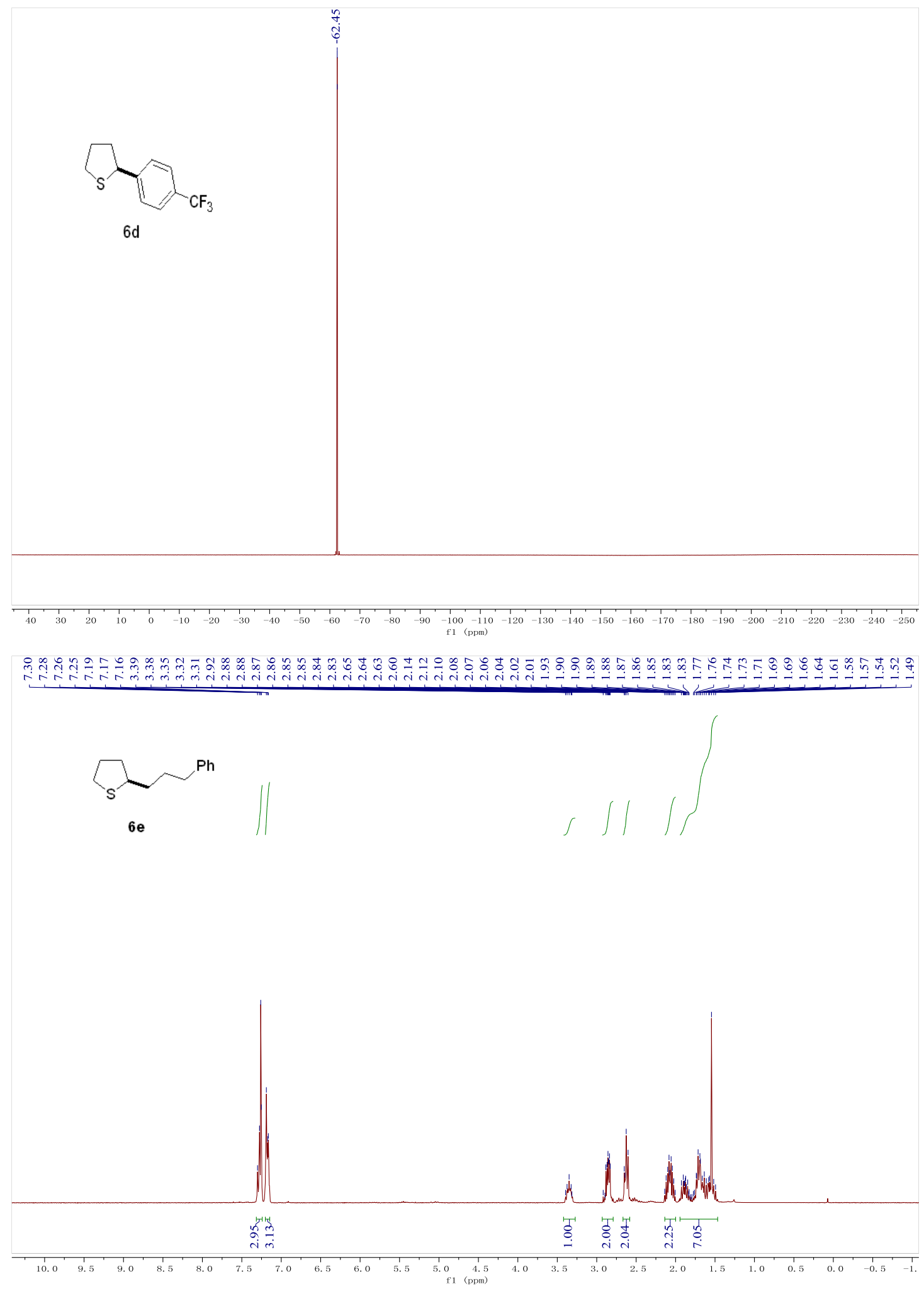




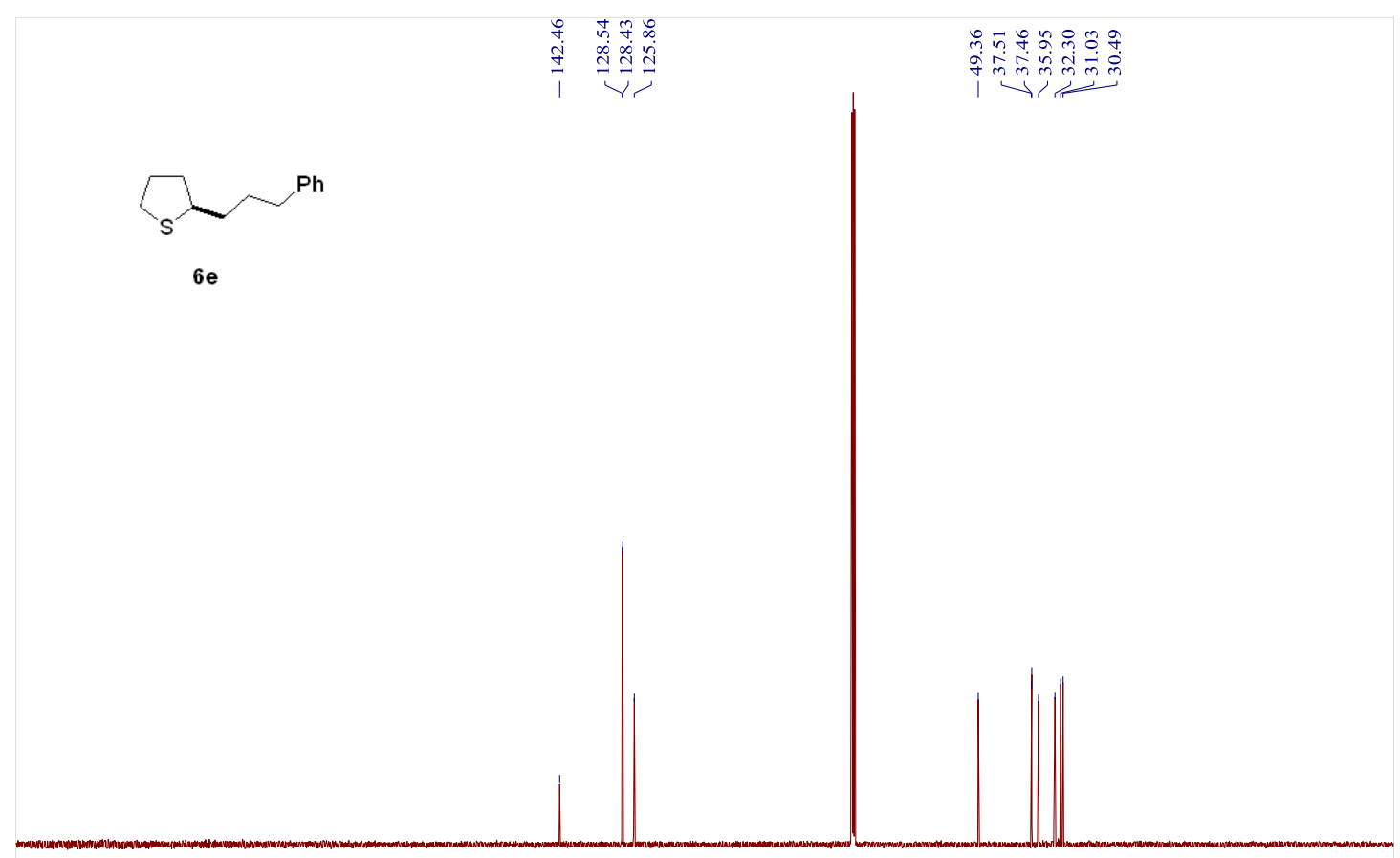

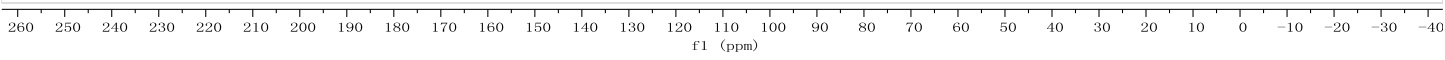

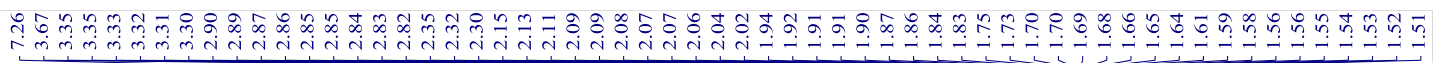<smiles>COC(=O)CCCC1CCCS1</smiles>

$6 f$ 

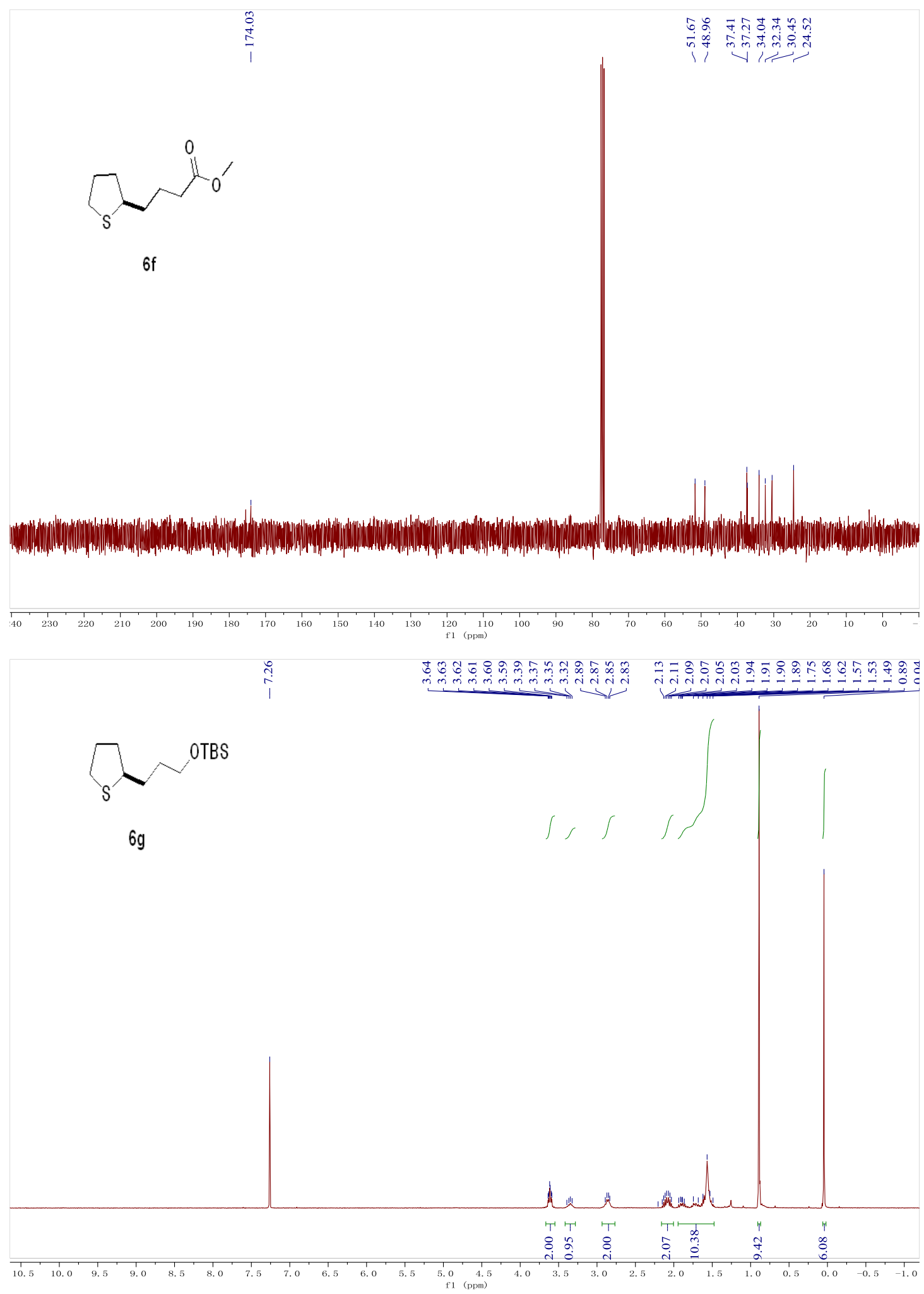


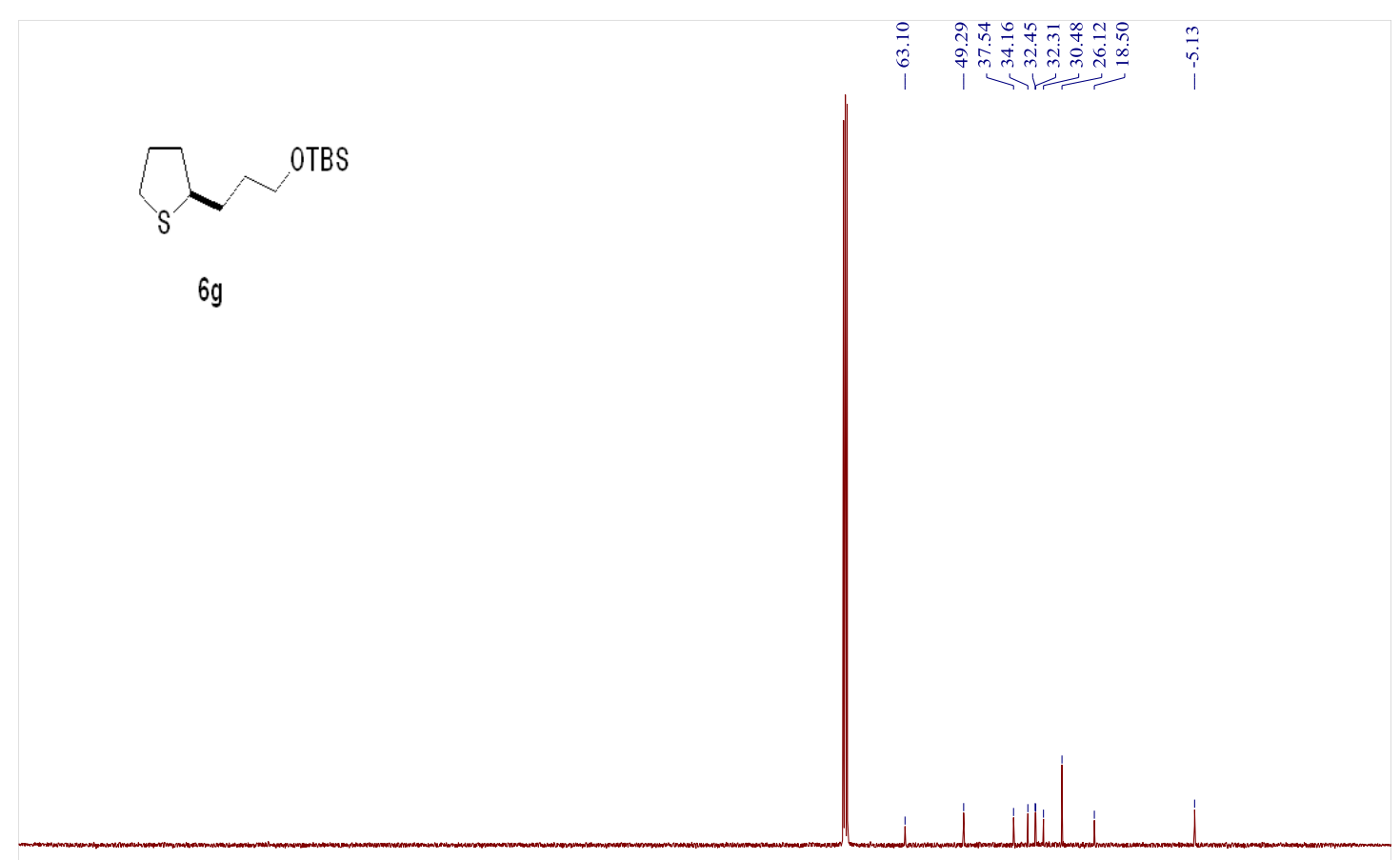

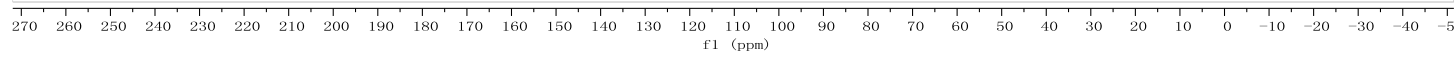

